\title{
Suicidal Ideation, Attempts, and Associated Psychosocial Risk and Protective Factors in American Indian Youth and Young
}

\section{Adults}

Karen Manzo

Follow this and additional works at: https://researchrepository.wvu.edu/etd

\section{Recommended Citation}

Manzo, Karen, "Suicidal Ideation, Attempts, and Associated Psychosocial Risk and Protective Factors in American Indian Youth and Young Adults" (2015). Graduate Theses, Dissertations, and Problem Reports. 6161.

https://researchrepository.wvu.edu/etd/6161

This Dissertation is protected by copyright and/or related rights. It has been brought to you by the The Research Repository @ WVU with permission from the rights-holder(s). You are free to use this Dissertation in any way that is permitted by the copyright and related rights legislation that applies to your use. For other uses you must obtain permission from the rights-holder(s) directly, unless additional rights are indicated by a Creative Commons license in the record and/ or on the work itself. This Dissertation has been accepted for inclusion in WVU Graduate Theses, Dissertations, and Problem Reports collection by an authorized administrator of The Research Repository @ WVU.

For more information, please contact researchrepository@mail.wvu.edu. 
Suicidal Ideation, Attempts, and Associated Psychosocial Risk and Protective Factors in American Indian Youth and Young Adults

\author{
Karen Manzo, MPH \\ Dissertation submitted \\ to the School of Public Health \\ at West Virginia University \\ In partial fulfillment of the requirements for the degree of \\ Doctor of Philosophy in \\ Public Health Sciences
}

\author{
Dissertation Committee \\ Robert D. Duval, Ph.D., Chair \\ Francine C. Gachupin, Ph.D. \\ R. Turner Goins, Ph.D. \\ Gerry R. Hobbs, Ph.D. \\ Alfgeir L. Kristjansson, Ph.D. \\ Joseph R. Scotti, Ph.D. \\ Department of Epidemiology \\ Morgantown, West Virginia \\ 2015
}

Keywords: American Indian, suicide prevention, minority health, adolescence, mental health, tribal college

Copyright 2015 Karen Manzo 


\title{
ABSTRACT \\ Suicidal Ideation, Attempts, and Associated Psychosocial Risk and Protective Factors in American Indian Youth and Young Adults
}

\begin{abstract}
Karen Manzo, MPH
Introduction. Research consistently shows that suicidal ideation and attempts are more prevalent among American Indian youth and young adults than youth and young adults in other racial/ethnic groups. Many of the associated psychosocial risk and protective factors are shared across racial/ethnic groups; however, for most of them, the prevalence is highest in American Indian youth. Research on the relation between suicide risk and culturally unique risk (e.g., childhood adversity, discrimination, parenting, unresolved grief, out-of-home placement) and protective factors (e.g., enculturation, cultural values, spirituality) in American Indian youth and young adults is limited. The goal of this research was to examine shared and culturally unique psychosocial risk and protective factors and coping strategies associated with suicide risk in American Indian youth and young adults.
\end{abstract}

Methods. Three interdependent cross-sectional studies were used to assess risk factors associated with suicidal ideation and attempts in Montana high school students using Youth Risk Behavior Survey (YRBS) data and to test a stress-coping model of suicide risk in American Indian tribal college students. In the first study, logistic regression was used to examine genderspecific individual-level risk factors associated with suicidal ideation/attempts for American Indian and White Montana high school students using pooled random sample secondary YRBS data. In the second study, logistic regression was used to identify gender-specific individuallevel risk factors associated with suicidal ideation and attempts in American Indian students attending Montana reservation and urban high schools using pooled volunteer sample YRBS data. In the third study, logistic regression was used to test a stress-coping model (situated within a cultural-historical context) to determine associations between suicide risk and life stressors, coping skills, enculturation, cultural values, resilience, and social support in American Indian tribal college students in Montana and Wyoming using primary cross-sectional survey data.

Results. The primary results of the first study showed that American Indian youth had significantly more suicidal thoughts and attempts than the White youth. However, within YRBS risk factors, they had fewer statistically significant risk factors compared to the White youth. Sadness/hopelessness was the strongest, and the only statistically significant, risk factor of suicidal ideation/attempts shared across all four groups. The unhealthy weight control cluster was a significant risk factor for the White youth and the American Indian girls; alcohol/tobacco/ marijuana use was a significant risk factor only for the American Indian boys. Findings for the second study showed that the prevalence of suicidal ideation and suicide attempts did not differ significantly between reservation and urban American Indian youth. However, regression analyses showed differences and similarities in significant risk factors for ideation and attempts for girls and boys across the locations. Fewer differences were noted for girls than boys. Sadness/hopelessness was the only statistically significant risk factor for suicidal ideation and attempts common for girls and boys in both settings. Some form of violence (partner victimization, lack of school safety) was significantly and positively associated with either ideation and/or attempts in almost all groups. Partner victimization was significantly associated with both ideation and attempts for girls, but was inconsistent for boys. Lack of school safety was significantly associated with suicide attempts across the four groups, but was not a significant risk factor for ideation. Findings from the third study in Montana-Wyoming tribal 
college students revealed low levels of past year suicide risk. Culturally unique stressors such as adverse childhood experiences, parenting difficulties, thinking of historical losses, and discrimination were significantly and positively associated with suicide risk. Gratitude, social support, participant boarding school attendance, and higher current income mitigated suicide risk.

Conclusion. Results using the YRBS data show important differences in risk factor categories across the racial/ethnic and reservation-urban groups for girls and boys and indicate directions for future research targeting prevention and intervention. Results from the tribal college setting show important areas for future research and have implications for socioeconomic, structural, familial, and culture-based intervention development. A comprehensive understanding of culturally unique life stressors and cultural buffers is important to inform effective interventions and reduce suicide-related morbidity and mortality in his population. 


\section{DEDICATION}

This dissertation research is dedicated to Gordon Belcourt, MPH (Blackfeet) and Cheryl Belcourt, BA (Mandan/Hidatsa), former and current Executive Directors for the Rocky Mountain Tribal Leaders Council ${ }^{1}$ located in Billings, Montana. Their support made this possible. I am deeply grateful.

${ }^{1}$ Formerly known as the Montana-Wyoming Tribal Leaders Council. 


\section{ACKNOWLEDGMENTS}

This dissertation research was funded through the West Virginia University School of Public Health (formerly Department of Community Medicine), the Rocky Mountain Tribal Leaders Council's Center for Mental Health Services and the Substance Abuse and Mental Health Services Administration grant (Award \#SM57380-01), and through pre-doctoral dissertation funding through the Ruth Landes Memorial Research Fund (Award \#1005521R).

This research was made possible by the leadership and staff of the Rocky Mountain Tribal Leaders Council and the Rocky Mountain Tribal Epidemiology Center. Also, the Montana Office of Public Instruction supported this research by providing access to data. Administration and faculty from the participating Montana-Wyoming tribal colleges viewed this research as important and their support made this research a reality.

For close to a decade, the staff and constituents of the Rocky Mountain Tribal Leaders Council and the Rocky Mountain Tribal Epidemiology Center have supported and encouraged my research. I am deeply grateful for their encouragement and teachings that made my research journey possible.

I would like to recognize and express my gratitude to the following:

The Rocky Mountain Tribal Institutional Review Board and the individual tribal and tribal college institutional review boards for approving this research.

Dr. Jera Stewart (Salish), former Research Director for the Rocky Mountain Tribal Leaders Council and Dr. Jennifer Giroux (Rosebud Sioux), former Director of the Rocky Mountain Tribal Epidemiology Center. Thank you for believing in me and for your continued support.

Drs. Robert D. Duval (dissertation chair), Francine C. Gachupin (Pueblo Jemez), and Alfgeir L. Kristjansson. Thank you for the time and effort you have invested after joining my committee in the past year.

Drs. R. Turner Goins, Joseph R. Scotti, and Hope M. Tiesman for your support while serving on my committee and Dr. Gerry R. Hobbs (statistician) for your mentoring and patience with my statistics questions.

Dr. Sarah S. Knox, thank you for challenging me and the time you spent mentoring me and honing my grant writing skills. And thank you for introducing me to epigenetics.

My fellow doctoral students, especially my friends Drs. Ruchi Bhandari, Loretta Cain, Brent Doney, and Tara Hartley. Thank you for your continued and lasting support.

Drs. Rose Pignataro and Allyson Kelley, my friends, colleagues, and readers. Thank you. Sincerely. May our friendship and work continue.

I am most grateful to my son and daughter-in-law, Matthew and Margie Tennant. Thank you for loving me and supporting me, especially during my travels. No words can express what you mean to me and my gratitude for creating my beautiful grandson, Raymond. Being near the three of you has kept me grounded and has given me many deeply treasured moments of joy. 


\section{TABLE OF CONTENTS}

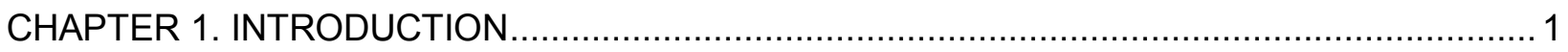

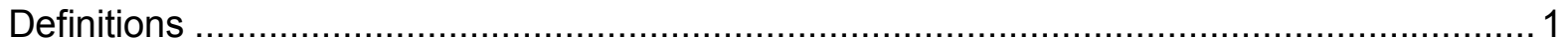

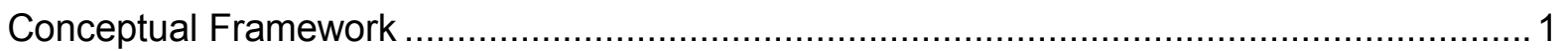

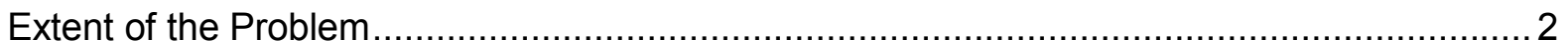

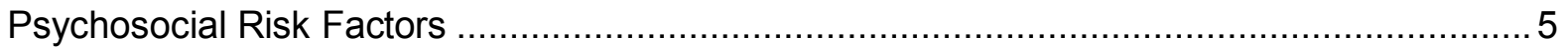

Psychosocial Risk Factors Unique to American Indians.............................................. 9

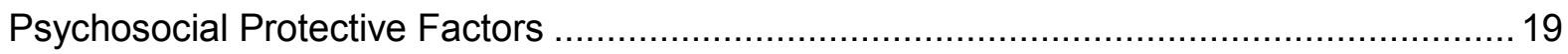

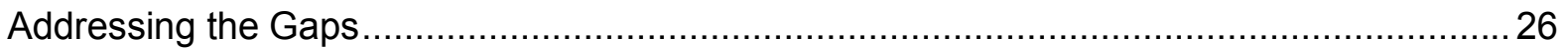

CHAPTER 2. A COMPARISON OF RISK FACTORS ASSOCIATED WITH SUICIDAL IDEATION/ ATTEMPTS IN AMERICAN INDIAN AND WHITE YOUTH IN MONTANA...............29

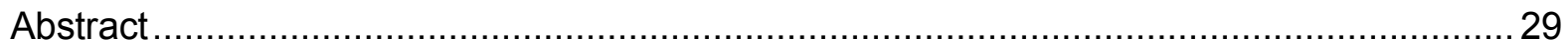

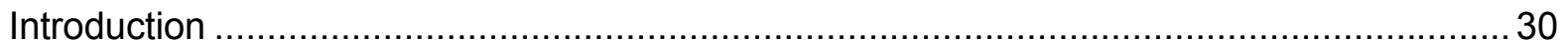

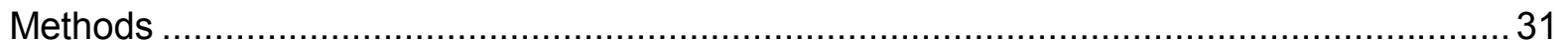

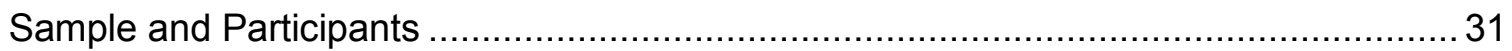

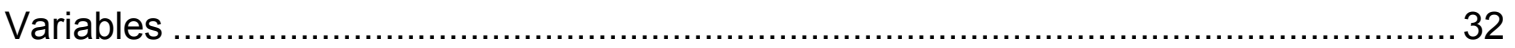

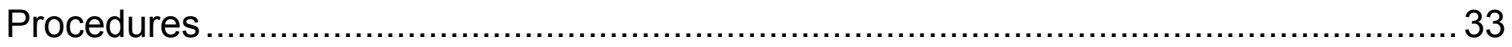

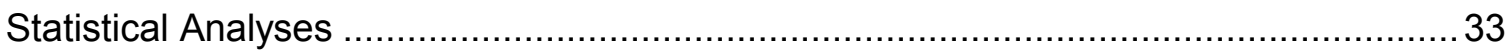

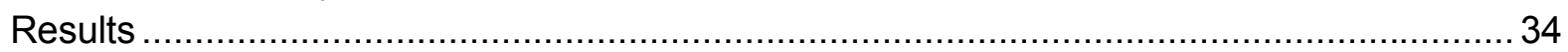

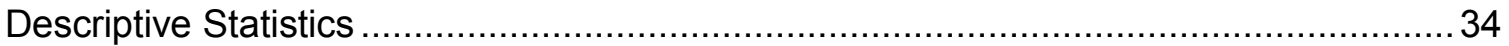

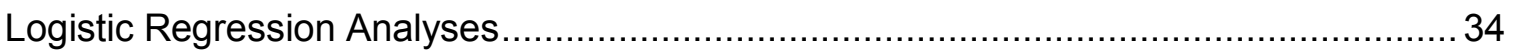

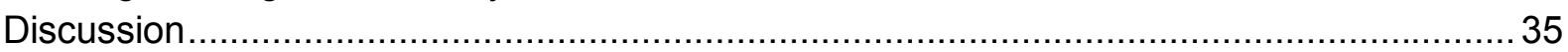

CHAPTER 3. RESERVATION-URBAN COMPARISON OF SUICIDAL IDEATION AND

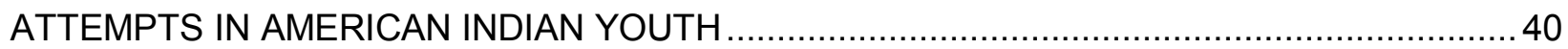

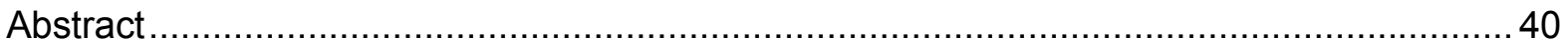

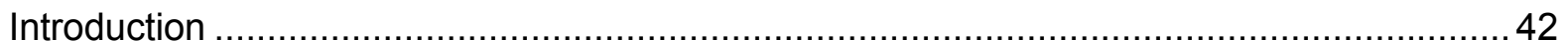

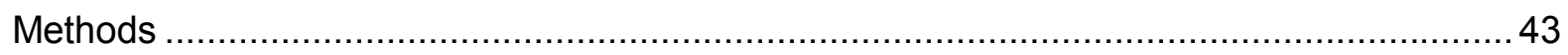

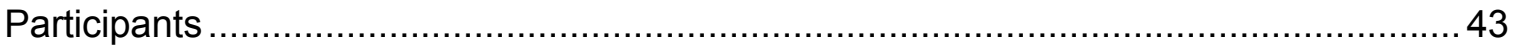

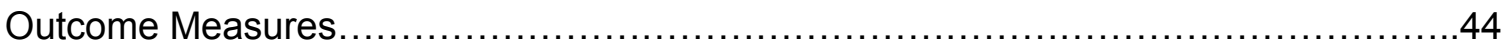

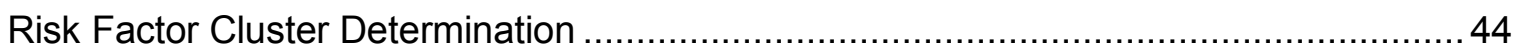

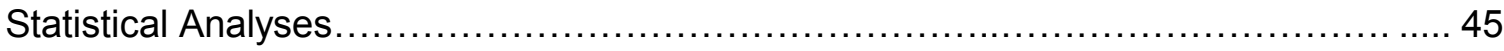

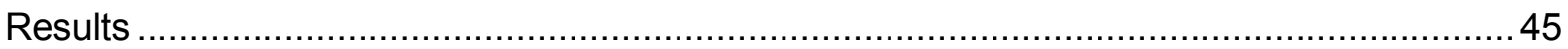

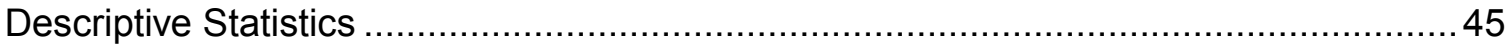

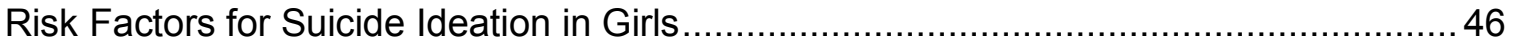

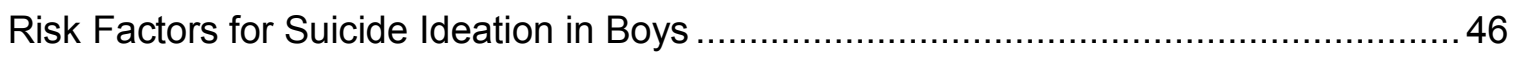

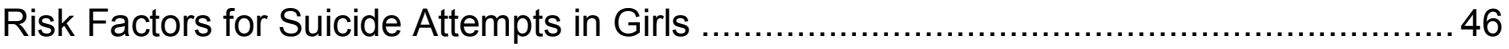

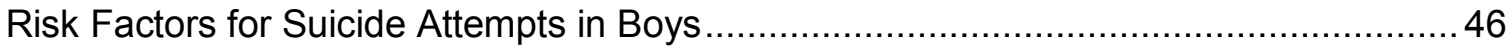

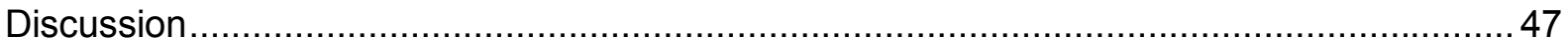


CHAPTER 4. TESTING A STRESS-COPING MODEL OF SUICIDE RISK IN A TRIBAL

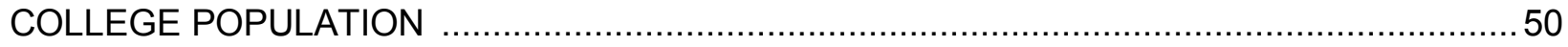

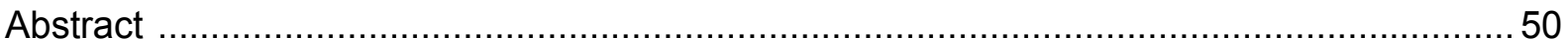

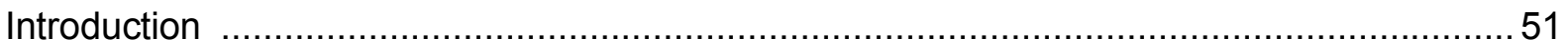

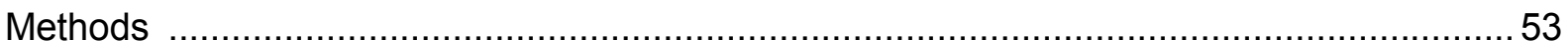

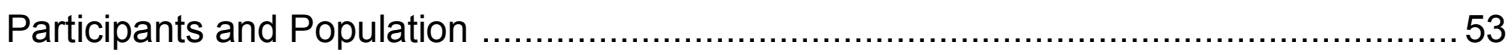

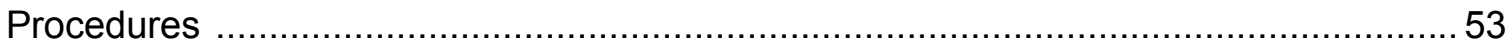

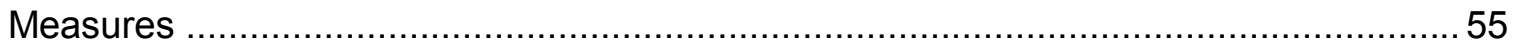

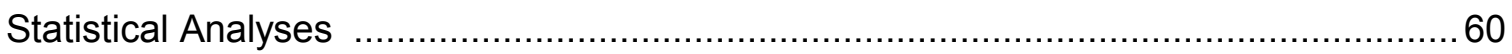

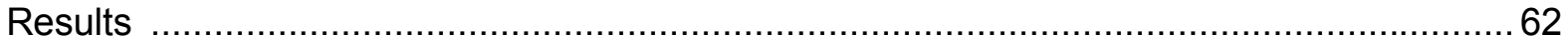

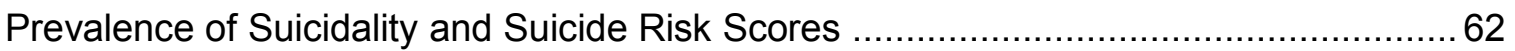

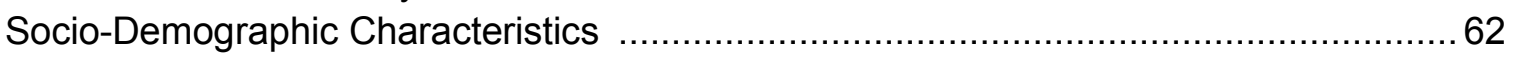

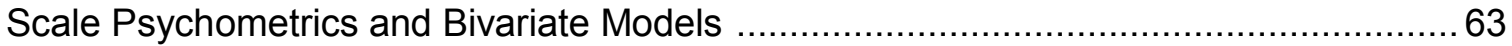

Principal Component Factor Loadings for Brief COPE Subscales ............................... 63

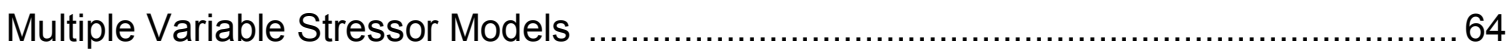

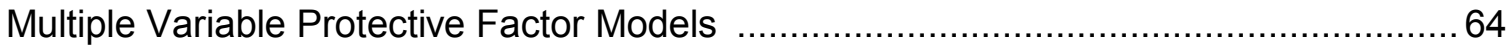

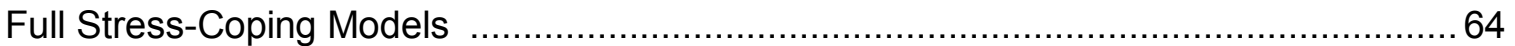

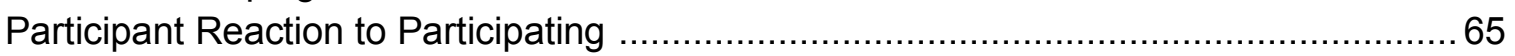

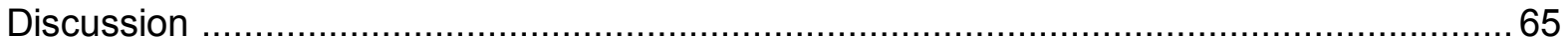

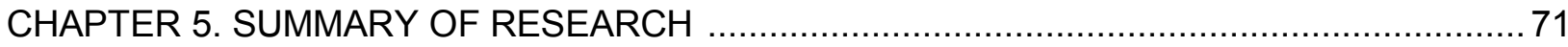

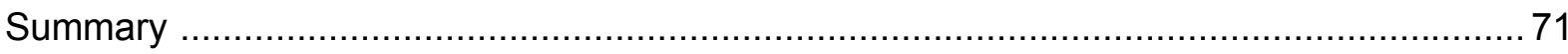

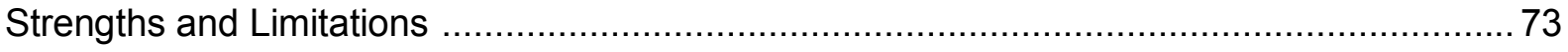

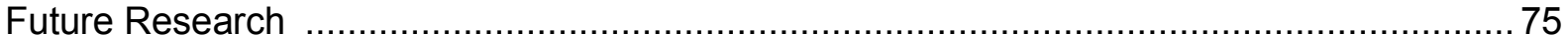

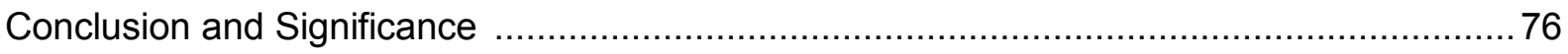

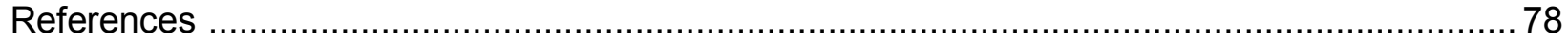

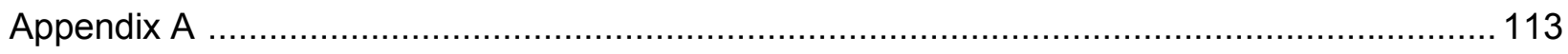

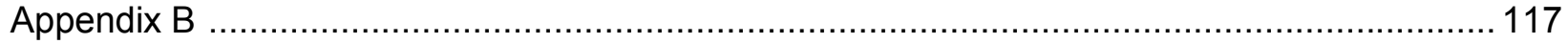

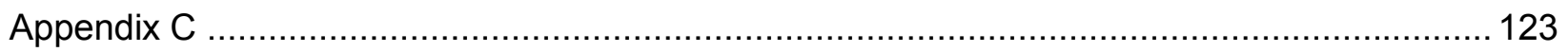




\section{CHAPTER 1. INTRODUCTION}

\subsection{Definitions}

For the purposes of this dissertation, suicide is defined as a death from a self-inflicted act. Suicidal ideation is defined as thinking about, considering, or planning for suicide. A suicide attempt is defined as a self-inflicted non-fatal act. Suicidality or suicide risk encompasses any or all of the above terms. Stressor is defined as a situation or event self-appraised as being aversive that elicits a physiological or psychological stress response (1). A traumatic stressor is an event that comprises actual or threatened death or serious injury, or alternative threats to an individual's well-being, as well as learning about the unexpected or violent death, serious harm, or threat of death or injury of a close associate or family member (2). Indigenous refers to any or all of the first peoples who live in the United States, Canada, Australia, or New Zealand. American Indian refers to Indigenous people living in the 48 contiguous United States (US). American Indian/Alaska Native refers to Indigenous people living in the lower 48 states and Alaska. First Nations refers to the Indigenous people of Canada. Different terminology is used to refer to Indigenous peoples in the literature. For the purposes of this dissertation, the term used in a particular study will be used when that study is referenced. The current research uses American Indian to refer to the dissertation research population. However, for brevity, the term Native may be used interchangeably with any of the Indigenous terms.

\subsection{Conceptual Framework}

Suicidality is a complex, universal phenomenon. Since Durkheim's seminal social integration theory (3), many models have been used to understand suicidality, including but not limited to: biomedical models [genetic $(4,5)$, epigenetic $(6-8)$, neurobiological $(9,10)]$; psychological models $(11,12)$ ]; life course developmental models (13-16); epidemiologic models (17-19); multi-level socioecological models (20-22); and, stress-related models (23-25). Individual level models are limited in that they ignore the social, cultural, and historical context of suicide. Developmental models are important to understand how age, gender, and identity formation affect suicidality $(15,16)$, but also are limited by their individual-level focus. Socioecological models consider the individual within the context of peers, family, culture, and 
society (20), which provides a framework for a more comprehensive understanding of suicidality among Native peoples.

Although Durkheim attributed suicide to social causes, and his suicide categories do not apply equally for men and women, the related, but independent social integration categories are useful for understanding Native suicide. Briefly and pertinent to Native populations, according to Durkheim, anomic suicide results from a lack of social regulatory influences wherein life crises disturb the collective order. As an example, how divorce or 'break-ups' may affect life equilibrium, especially in men $(26,27)$. His egoistic category states that suicide results from a lack of social integration. In Native communities, this is reflected in historical events that radically altered the social structure, including assimilation policies, such as mandatory boarding school attendance and urban relocations, as well as the current exorbitantly high rates of unemployment and incarceration in Native communities. This social disintegration has disrupted family relationships and kinship structure, inhibiting the generational transmission of language, traditional parenting skills, and cultural practices and values, thereby weakening the collective conscience deemed important by Durkheim for strong social integration $(28,29)$.

As a conceptual framework, this dissertation work uses an Indigenist stress-coping model that is situated within a unique socio-cultural, historical context (24, $25,30-33)$. Stresscoping models have been used to conceptualize suicide risk in the general population $(34,35)$. A stress-coping model for suicide risk has not been tested in this population, although a stresscoping model has been conceptualized for Native health overall $(24,25,36)$. This framework also incorporates developmental aspects, because coping strategies are often age- and genderas well as culture-based (37-40). Similarly, a stress-vulnerability model has been used to conceptualize suicidality in several populations $(23,41,42)$, including college students (43). However, stress-vulnerability models focus on vulnerabilities only. The Indigenist stress-coping model was selected as a conceptual framework for this research because it focuses on cultural strengths that may serve as stress buffering mechanisms. Focusing on cultural strengths, as opposed to a deficit, risk factor model, is a life- and hope-affirming approach $(21,44)$. The following presents a comprehensive review of the literature, followed by how gaps in the literature are addressed through the research specific aims.

\subsection{Extent of the Problem}

Suicide deaths, suicidal ideation, and non-fatal suicide attempts are a worldwide public health problem with extreme heterogeneity between and within countries $(29,45-47)$. Suicide is 
a leading cause of death in the US, with highest rates consistently found in Alaska, Montana, and Wyoming (http://www.suicidology.org). Prior to contact with Europeans, suicide was rare in Indigenous communities $(48,49)$. Many tribal groups had, and still have, explicit cultural beliefs prohibiting suicide $(26,50)$. Today, however, rates of suicide are two to four times higher among Indigenous peoples worldwide than their national counterparts (51-54). Indigenous suicide rates also vary greatly within and across countries $(29,55-59)$. In contrast to the general population where suicide deaths occur most often in older adults $(60,61)$, Indigenous suicide deaths occur most frequently in those under 25 years and are especially high in ages $20-24$ years $(62,63)$. One Southwest tribe reported age-adjusted suicide rates of 40/100,000; however, the rate reported for 20-24 year olds was 152/100,000. These are 4 and 12 times the rates for respective age groups in the US as a whole (62). Compared to non-Native contemporaries, American Indian males 15-19 years of age are two to four times more likely, and American Indian females of that age are 11 times more likely, to commit suicide than corresponding US youth (64); yet these rates are thought to be underestimates. Overall, the rate of suicide is lower among college students than their non-college peers; however, suicide is the second leading cause of death among college students (65-67). Suicide death data are not available for American Indian college students.

Suicide deaths in the US are seriously undercounted and suicide mortality appears vulnerable to differential misclassification according to decedent race/ethnicity (68). The Indian Health Service estimates that American Indians are misrepresented by an average of $11 \%$ on state death certificates (69). This racial/ethnic misclassification appears to be most problematic for suicide deaths (70), contributing to a potential underestimation of suicide as a public health problem in this population. American Indians also appear to have a higher rate of undetermined injury deaths than other racial/ethnic groups (71). These data are important for suicide prevention efforts, because research shows a strong link between injuries of undetermined intent and suicide in other minorities (72-74). For example, data show greater potential for the misclassification of suicide as injury deaths of undetermined intent for African Americans than Whites and for females of both racial/ethnic groups (72).

Suicidal ideation and suicide attempts are both significant predictors for risk of a completed suicide $(18,75)$. Like suicide deaths, suicidal ideation and attempts also vary by gender, age, racial/ethnic group, geographic location, and cultural subgroups. Worldwide, women attempt suicide at least three times more often than men (45). Overall, suicidal ideation and attempts occur most commonly among younger people, whereas suicide deaths occur most commonly among older adults $(14,61)$. Overall, suicidal ideation and attempts are much higher 
among Indigenous youth than youth from other racial/ethnic groups. This is true for the US as well, where suicidal ideation and attempts are significantly higher for American Indian youth ages 15-19 years (especially girls) than other US racial/ethnic groups (76-78).

Among Indigenous youth, several studies have reported prevalence of suicide attempts and a limited number have reported prevalence of suicidal ideation. In the US, a national study found that prevalence estimates for suicidal ideation and suicide attempts, respectively, are highest among American Indian (23.1\%; 16.4\%) and Hispanic youth (16.5\%; 10.1\%), and lowest among African American youth (12.3\%; 7.8\%) (78). Another national study found significantly higher suicidal ideation and suicide attempts for American Indian youth than either Asian, African American, Hispanic, or White youth (79). A study of urban youth showed suicide plans and attempts were two and three times higher for American Indian than White youth (80). Similarly high estimates were noted in two separate nation-wide studies of youth from reservation-based schools, where $22 \%$ of the girls and $12 \%$ of the boys reported ever attempting suicide $(81,82)$. Also, a study of schools funded by the Bureau of Indian Affairs reported that $19.3 \%$ of girls and $12.2 \%$ of boys had ever attempted suicide (83).

Regional and tribal variations also exist. This variation is to be expected because American Indian tribes are culturally heterogeneous (language, kinship structure, gender roles, conceptualization of death) and have different assimilation experiences (84-86). However, Novins and colleagues found no significant difference in past month suicidal ideation among high school youth from three culturally distinct tribal areas (Southwest [2.3\%], Northern Plains [5.0\%], Pueblo [3.8\%]) (86). However, cultural heterogeneity is evident in this study in that none of the statistically significant risk or protective factors for ideation were common across the three tribal areas. In Hawaii, Native Hawaiian youth reported significantly higher suicide attempts $(12.6 \%)$ than non-Native Hawaiian youth (9.6\%) (76). This is also true for Oregon, where American Indian youth (10.5\%) reported twice as many past year attempts as White youth $(5.3 \%)(87)$.

The sparse epidemiological suicide data for American Indian college students show that they report higher suicidal contemplation than college students overall $(15 \%$ vs. $9.5 \%)(88,89)$. In contrast with the youth and young adults, population-based data show that American Indian adults were significantly less likely to report lifetime suicidal ideation compared to National Comorbidity data (90). However, the same study also reported regional differences in that Northern Plains tribal members were significantly more likely to report lifetime suicide attempts than either Southern Plains tribal members or the general population. 


\subsection{Psychosocial Risk Factors}

Like suicide deaths, suicidal ideation and attempts are complex phenomena with many potentially modifiable psychosocial risk and protective factors (91). Many of these risk factors are shared across racial/ethnic groups; however, the prevalence of most of these risk factors is highest in American Indian youth (78). As with other racial/ethnic groups, American Indian girls report attempting suicide almost twice as often as American Indian boys (82). Research shows a direct relation between gender and suicidal ideation and attempts, as well as various individual-level psychosocial risk factors. Risk factors shared among racial/ethnic groups include: previous suicide attempt (82); poor mental health (11, 82, 92-94); co-morbid psychiatric disorders $(11,95)$; minority sexual identity/attraction $(82,92,94,96-98)$; exposure to suicide (82, 92, 94, 99); bullying (94, 100); and life stress, such as child physical and sexual abuse $(101,102)$, intimate partner $(103)$ and dating violence $(97,104)$, and sexual assault $(87,105)$. Each of these factors may be exacerbated by unhealthy coping responses (106), as well as enabling factors such as access to firearms (107-111) and substance use $(82,92,94,112$, 113). Socio-environmental factors associated with suicidality are also shared across racial/ethnic groups, such as family structure $(92,114)$, family substance abuse $(99,115)$, and poverty $(11,116,117)$. However, research in other populations indicates that social fragmentation may play a more important role than poverty $(28,117-119)$.

\subsubsection{Psychiatric Disorders}

The strongest predictor for suicidality is the presence of psychiatric disorders. In particular, depression consistently shows the strongest independent risk for suicidal ideation and attempts $(11,120-123)$, especially when comorbid with anxiety or disruptive disorders. One example is the Great Smoky Mountain Study (GSMS). Researchers randomly selected and screened 4500 of the 11,758 American Indian and White youth (aged 9, 11, and 13) in 11 counties in the southeastern US for psychiatric symptoms. Data from the GSMS reveal strong significant associations for suicidality and depression $(O R=21.7)$ and depression comorbid with general anxiety disorder (OR $=50.2$ ) or disruptive behavior disorder (OR $=5.8$ ), even after controlling for age, gender, race, poverty, and number of psychiatric symptoms (11). In contrast, associations with suicidality and substance abuse have been mixed across studies $(11,124)$. Interestingly, the GSMS showed an inverse association for illicit drug use, and no significant association with alcohol use, and suicidality (11), while other studies with youth have shown an association between alcohol and suicide attempts. For example, a study of Navajo youth found 
an association between weekly hard liquor use $(O R=2.7)$ and suicide attempts, after adjusting for age, gender, child physical/sexual abuse, exposure to suicide, mental health, and other substances (124). The researchers also noted a linear trend for frequency of hard liquor use and increasing prevalence of suicide attempts.

\subsubsection{Traumatic Experiences}

Suicide risk also is associated with experiencing adversity as a child, especially cumulative adversity (125). Childhood adversity is associated with many adverse health and social outcomes, including suicide-related risk factors such as depression (126), intimate partner violence (127), and substance abuse (128-131). The Adverse Childhood Experiences (ACE) Study examined associations between potential adverse experiences (emotional, physical, and sexual abuse; battered mother; household substance abuse and mental illness; parental separation or divorce; and, household member incarceration) that occurred during the first 18 years of the participant's life and various health outcomes in adulthood (132). The associations were strong and often showed a linear relation with cumulative adversity. Those reporting any individual ACE were two to five times more likely to attempt suicide than those reporting no ACE, even after adjusting for age, gender, race, and education. The risk for attempting suicide increased substantially with an increasingly higher number of reported ACE. In addition, the linear, exposure-response relation for cumulative adversity was twice as strong in those who attempted suicide during childhood/adolescence as compared to those who attempted suicide in adulthood. This graded relation was consistent across four birth cohorts (133).

A separate study of American Indian adults from seven tribes found significant associations between various ACE and alcohol dependence (134). This study found statistically significant tribal and gender differences in the prevalence of ACE and alcohol abuse/dependence. There were also gender differences in which ACE were significantly associated with alcohol dependence, especially for those reporting multiple experiences. For women, sexual abuse and boarding school attendance were significantly associated with alcohol dependence, while experiencing both physical and sexual assault as a child was significantly associated with alcohol dependence in men. Childhood adversity has also been linked to early initiation of substance use in Native youth (135), who may not have had time to develop effective coping strategies, such as problem solving skills. Although severe childhood adversity can negatively affect psychosocial development, the effects can be mitigated by 
having a close bond with an effective parent, parental surrogate, or mentor, such as a teacher or other community member (136).

Acute and chronic stressors are pervasive in the lives of American Indians who are exposed to an exorbitant number and type of traumatic experience over the life course. The American Indian Services, Utilization, Psychiatric Epidemiology, Risk and Protective Factors Project (AI-SUPERPFP) was the first large, population-based, socio-psychiatric epidemiology study in American Indians from two culturally distinct tribal areas (Northern Plains, Southwest) (137). Their socio-epidemiological analysis of trauma revealed differences and similarities between the Southwest and Northern Plains tribal areas (138). For example, trauma prevalence differed by type of trauma, tribal area, and gender; however, exposure to suicide was similar for men and women from both tribal areas. As would be expected, women of both tribal areas reported significantly more interpersonal trauma, rape, molestation, and intimate partner violence than men. Men of both tribal areas reported a significantly higher prevalence of nonsexual physical attacks. Those with higher education levels were significantly more likely to experience or be exposed to trauma for either tribal area. Living in poverty showed a significant inverse association with some trauma categories, but for the Southwest tribal area only. Because this is contrary to the general trauma literature (139), minority stressors may be a factor. Research has also shown that higher education levels, while protective against suicide in White men, increased the risk of suicide for African American men (140). Further research is needed to determine which factors play a role in this racial/ethnic inconsistency. For example, perceived discrimination, level of cultural integration, or cultural identity may play a role. Studies also have shown a link between traumatic experiences and substance abuse for youth and adults (141-144).

The AI-SUPERPFP group also examined associations between psychiatric disorders and suicidal behaviors (Northern Plains tribal area only) (121). Consistent with the literature, women and young adults reported significantly higher suicidal behaviors. Female gender, depressive disorder, posttraumatic stress disorder (PTSD), substance abuse/dependence, and violent ideation/aggression were significantly associated with both suicidal ideation and attempts. After adjustment for the other variables, depressive disorder $(O R=5.5)$ and violent ideation/aggression $(\mathrm{OR}=4.3)$ showed the strongest association with suicidal ideation; suicide attempts were more likely among those reporting depressive disorder (OR = 3.7) or PTSD (OR = 2.9). In a second suicide study, this group compared the association of mental disorders and traumatic events with suicidal ideation and attempts in two tribal areas with a general populationbased sample (90). Data showed similar significant risk factors across the three 
groups with minor exceptions. There was a significantly stronger association between ideation and being in an accident or experiencing other unexpected trauma, and a significantly lower association between attempts and anxiety disorders in the Northern Plains group compared to the other two groups.

\subsubsection{Discrimination}

Discrimination is a multifaceted, pervasive minority life stressor $(145,146)$. It has been linked to multiple physical, mental, and social health consequences (147-151), including poor mental health $(150,152-163)$, suicidal ideation and attempts $(113,164-166)$, substance abuse $(151,162,167,168)$, and unmet health care needs (169-171). Discrimination appears to affect men more than women (172). A limited number of studies have examined the effects of discrimination on Indigenous health. Among Native Hawaiians, high perceived discrimination and identification with mainstream culture were independently associated with hypertension (147). Associations were found between discrimination and poor mental health outcomes in Australian Aborigines (173). Similarly, discrimination has been associated with bullying and poor health in Norwegian Sami $(174,175)$. Discrimination has been recognized as a risk factor for suicidality in minorities for some time (164-166), including American Indians (113, 176); however, the research in American Indian populations is limited and shows conflicting results. One study, using a 10-item measure of discrimination, found a significant association between perceived discrimination and suicidal ideation in reservation youth controlling for gender, enculturation, negative life events, self-esteem, and substance use (113). In contrast, two studies using a single-item measure of perceived discrimination found no significant association with suicidality in American Indian youth $(82,99)$.

Discrimination also has been linked to substance use in youth and adults $(167,168)$, especially in boys, where the data show discrimination leads to increased use of substances over time (167). It also has been linked with substance abuse in Native youth $(177,178)$. As with childhood adversity, discrimination is associated with early initiation in substances in Native youth (177). Youth may not be developmentally capable of choosing more effective coping strategies or they may not have access to them $(178,179)$. Participation in traditional activities (180), healthy coping responses $(181,182)$, and high self-esteem (183) appear to lessen the negative health effects related to discrimination. 


\subsection{Psychosocial Risk Factors Unique to American Indians}

Governmental policies which have adversely affected the health and well-being of Indigenous people worldwide provide the socio-historical context to frame these experiences. This is frequently referred to as historical or intergenerational trauma $(30,184-186)$. The concept of historical trauma is commonly described as a soul wound or as unresolved grief that is thought to manifest as physical, psychic, and spiritual suffering, the effects and reenactment of which are believed to be transmitted from generation to generation $(30,32,187)$. It characterizes the accumulated and collective suffering, loss of a way of life, and cultural oppression that spans generations and includes many human rights violations. Examples of historical trauma include forced removals, horrendous living conditions with inadequate and insufficient food, forced acculturation, forced sterilizations; banning of religious practices and languages; boarding school abuses and deaths; disproportionate foster care placement; disrupted family and cultural attachments and identity; and present day racism and discrimination $(30-32,36,83,178,188-192)$. All of these are believed to contribute to contemporary American Indian health disparities. The bulk of the historical trauma research has been conducted among Jewish Holocaust survivors and their children (193-199); however, empirical research is beginning to operationalize historical trauma among American Indians $(105,178,200)$.

\subsubsection{Assimilation Period}

In the early history of the US, tribal nation status went from full sovereignty to constitutionally defined "domestic dependent nations (201)." Policies established during the Assimilation Period (1887-1953) were deliberate and concentrated efforts to impose individualistic and Christian values on American Indians (202); less stringent efforts were made prior to this period, for example, through the Civilization Fund Act of 1819 (185). The federally funded church- and government-run boarding school system was a unique aspect of assimilation policy. The boarding school era is an especially disturbing part of American Indian history because it separated Native children from their families and culture and placed them in sometimes unspeakably abusive conditions (203). Actions ranged from misplaced benevolence by White women wanting to provide "proper" child care and education for the Native children (204), to acts that today could be classified as genocide by the standards of the 1948 Genocide Convention (202, 205). Specifically, Article 2 of the Convention describes genocide as acts committed with intent to destroy, in whole or in part, a national, ethnical, racial or religious 
group, including . . f forcibly transferring children of the group to another group. This was epitomized by the ideological foundation of boarding school originator, Richard Pratt (former prisoner of war warden): Kill the Indian, and save the man (206).

In the 1870s, as slavery ended and African Americans and new waves of immigrants were being assimilated into American society, the US government instituted off-reservation boarding schools for the express purpose of culturally assimilating American Indian children. Placed in schools that were often intentionally far from their home reservations, the children were forced to deny their tribal identity, culture, traditional practices, and language, which they were punished for speaking, at times severely. Also during this time period, traditional ceremonies were banned and assimilation policies forced Christian religion and ideals on Native populations. This was reinforced in the boarding schools through military-style discipline. For example, children lived in prison-like settings, wore uniforms, and were required to march throughout the day. As punishment, they could be imprisoned in the guardhouse, placed in isolation, deprived of food, or forced to run the "belt-line," a line of whippings often led by fellow students $(206,207)$.

Some parents wanted their children to have an education and willingly entrusted their children to the care of school personnel. However, many parents were coerced into sending their children, and many children were traumatically separated from their families, causing a burgeoning distrust of the conditions at the schools. In extreme cases, children were stolen, hog-tied, or put in cages to be transported to the schools (208). Due to mounting distrust, many parents hid their children to protect them, went without rations, or went to jail for refusing to allow their children to attend (209). Many students rebelled by running away from the schools, setting fires, conducting clandestine ceremonies, and engaging in passive resistance. The results of the unsanitary, disease-ridden, and overcrowded conditions and malnourishment led to many deaths. Medical care and conditions were so poor that each school had a graveyard $(210,211)$. The schools were understaffed, primarily by single Christian women who were themselves separated from their families. The teachers were underpaid and unaccredited. Children were segregated by age and gender. Many of the young children who required more care did not receive adequate attention. Many siblings had little contact with each other. Symbolically laden changes occurred upon entering the boarding school. The children's hair was cut (a symbol of grief for many tribes), their names were changed or they were given a number, and at times, their traditional clothes were destroyed (209).

The children at the boarding schools suffered intellectual, cultural, emotional, physical, and sexual abuse at the hands of staff, clergy, and older children. Their education was 
rudimentary and manual labor-focused, often teaching menial skills that were not useful for reservation life or for securing employment once they left the boarding schools (206). The emphasis for boys was farming and manual labor, at times coffin making for children who had died. The emphasis for girls was domestic service and Victorian moral standards (206). Because the schools were severely underfunded, child labor built and furnished many schools. The children labored in gardens and dairies, in many cases having no access to the food produced. The "outing program" was another source of unpaid child labor. Rather than send the children home during the summers where they would be exposed to their families, language, and traditions, children were involuntarily sent to live with and labor for White families during the summer months $(209,212)$. The boarding school movement occurred during a time when the US was only beginning to institute compulsory education laws (185). Ostensibly, requiring the average American child to attend school protected them from child labor exploitation that was common in the US until the institution of the Fair Labor Standards Act in 1938. In reality, in the case of the average Native child, compulsory education and child labor often went hand-inhand.

\subsubsection{Reorganization Period}

Periods of policy reform began after the 1928 Meriam Report (211); however, few changes were enforced. Published four years after the Indian Citizenship (Snyder) Act of 1924 (185), the report strongly criticized the boarding school system and brought the "grossly inadequate" boarding school conditions to light. Some of the recommended changes included improving the children's diet, decreasing the heavy work-load, and increasing the number of onreservation public day schools. The Meriam Report also suggested improving the crowded conditions by keeping children at home until adolescence to not harm the children's development by taking them from their parents at a young age. Although the assimilation period officially ended with the enactment of the 1934 Indian Reorganization Act (the Indian New Deal), World War II interrupted changes from taking place (213). During this time period, more emphasis was placed on reservation, than off-reservation, boarding schools. After the Termination Act of 1953, the government encouraged public school attendance; however, 93 boarding schools were still in operation (214). At this time, 51,000 (26\%) American Indian children attend boarding schools as opposed to $80 \%$ of American Indian children circa 1900 (215). In some cases, boarding schools became the norm with parents and communities expecting that the children would attend the "Indian schools," instead of the "White" public schools $(208,214,216)$. 
Forty years after the Meriam Report, the 1969 Kennedy Report Indian Education: $A$ National Tragedy: A National Challenge found similar failures with the boarding schools, including inadequate funding, deficient education, and a rigid, impersonal environment (217). Discriminatory practices continued into the 1960s, where students at one Montana reservation boarding school were segregated by blood quantum, (i.e., skin color; personal communication), illustrating on-going assimilationist attitudes. Although limited in number, there have been a few success stories, as well. For example, Jim Thorpe excelled in sports, and Charles Alexander Eastman, MD and Gertrude Simmons Bonnin were two of many who used their boarding school education to advocate for American Indian civil rights (212).

\subsubsection{Self-Determination Period}

After the institution of the Indian Self-Determination and Education Acts of the 1970s and the Indian Child Protection Act of 1990, tribes were able to assume more responsibility for their children's education, including stringent background checks for teachers and staff. At present, boarding schools are no longer mandatory; however, many American Indian children still receive their education in boarding schools. Although most American Indian children currently attend public day schools about 17,000 currently attend boarding schools (218), which can be a protective environment for many at-risk youth (77). Many now feel an attachment to and ownership of the "Indian" schools. Reasons for attending contemporary boarding schools include remote home locations, concerns with discrimination at local schools, youth in crisis, delinquent behavior, and to escape abusive home environments $(77,208,212,219)$.

During this time period, tribes also began establishing tribal colleges. The Tribally Controlled Community College Assistance Act of 1978 and the Higher Education Act affirmed the inherent right of tribes to educate their citizens (220). To be accredited, tribal colleges follow the principles of the Association of Governing Boards. However, tribal colleges are unique in that they also promote the development of cultural integrity, identity, and traditional values through courses in American Indian studies, tribal government and law, tribal history and languages, and traditional arts, spirituality, and medicine. There are currently 36 accredited tribal colleges nation-wide with approximately 20,000 students (221).

\subsubsection{Out-of Home Placement}

As boarding school placements were lessening, a disproportionate number of American Indian children were being placed in foster care or adopted out, usually with non-Native families. During the 1960s, American Indian children in Montana were 13 times more likely to be placed 
in foster care than non-American Indian children in the state (208). Traditional child rearing practices and kinship networks were misunderstood and judged as "permissive" and inferior to White middle-class values. A combination of discriminatory standards, "drunken Indian" stereotypes, due process violations, parents not understanding their legal rights, and monetary incentives for foster parents continued the disruption of families for $25-35 \%$ of American Indian children during the 1960 s and 1970 s $(208,222)$. This is in comparison to $0.5 \%$ of US children overall.

The Indian Child Welfare Act of 1978 established minimum standards and safeguards to protect the best interests of the American Indian children (222). Application of the federal law varies across states $(223,224)$, several of which have created an "existing Indian family" exception that allows state courts to decide the ethnic identity of a mixed race child (225). Currently, more than 10,000 American Indian/Alaska Native children are in foster care across the US (226). Nationwide, American Indian/Alaska Native children are more than twice as represented in foster care as children in the general population (227); the disproportion is greater for Northern Plains states where the number of Native children in foster care can be three to four times higher than in the general population of their respective state. For example, although Native children represent $10 \%$ of the child population of Montana, they represent $33.6 \%$ of the children placed in foster care in that state. This disproportion has remained constant since 2000 (227).

\subsubsection{Empirical Research to Date}

There is a paucity of research or theory related to the impact of boarding schools, whether among the privileged, or among American Indians (228). Even when socially accepted, boarding school attendance can be traumatizing for any child, especially children who leave their primary caretaker during the latency developmental stage, around age 5 to adolescence. From clinical observation, Schaverien has proposed a Boarding School Syndrome to describe the "cluster of learned behaviors and discontents" resulting in disrupted parent and sibling relationships and intimacy problems (228). She suggested these losses result from early childhood boarding school attendance and homesickness, and the bullying and sexual abuse that take place in the schools. American Indian boarding schools and the traumas and life stress associated with the federal boarding school system and out-of-home placement are ubiquitous in the lives of American Indians and pose unique health risks compared to other racial/ethnic groups in the US. 
Baseline data from a Canadian First Nations longitudinal health survey illustrate the damaging health effects of boarding school attendance (229). About $18 \%$ of attendees reported lifetime suicide attempts. Although this was not significantly different from non-attendees, there appear to be generational effects. For example, adults (ages 18+) who had at least one parent attend boarding school reported significantly more suicidal ideation (37\% vs. $26 \%$ ) than adults whose parents did not attend a boarding school. Significantly more suicide attempts (20\% vs. $13 \%$ ) were reported among adults whose grandparents attended boarding school compared to those whose grandparents did not attend. Youth (ages 12-17) whose parents or grandparents attended also were more likely to report suicidal ideation/attempts than youth of non-attendees, illustrating the potentially negative generational effects of the boarding school system.

Additionally, many of the attendees reported experiences that are associated with suicidal ideation and attempts, including witnessing the abuse of others $(71.5 \%)$, being sexually $(32.6 \%)$ or physically (79.2\%) abused, or being bullied (61.5\%). Research shows a significant inverse association between boarding school attendance and self-perceived health, even after adjusting for education and employment, which were both positively associated with self-perceived health (230).

Significantly more experience with interpersonal trauma (direct or witnessed) was reported among US boarding school attendees, as well (141). Gender differences are evident. Results from one study showed that men attendees reported having experienced more physical and sexual abuse and punishment for speaking their language than did women attendees (231). Data from another study show that boarding school attendance was associated with alcohol dependence in American Indian women, but not American Indian men (134). A study of general and culturally relevant stressors showed a significant association between boarding school attendance and depressive symptoms after adjusting for childhood adversity and thinking about historical losses, but not negative adult life events (232).

A cohort of youth and young adults who had at least one parent who attended boarding school reported significantly more sexual assaults and suicide attempts than those whose parents were non-attendees $(49,105)$. In addition, a recent study of First Nations adults found a significant association between boarding school attendance and being abused, but not suicidal thoughts or attempts (233); however, attendees who had a history of abuse were significantly more likely to have reported suicidal thoughts or attempts. Having a parent or grandparent attend boarding school was significantly associated with a history of abuse, suicidal thoughts, and attempts for First Nations adults who did not attend boarding school themselves, although 
there were no significant generational associations for participants who had themselves attended boarding school.

Foster care placement and adoption also can adversely affect health. In the general population, research shows a significant, positive association between foster care placement and depressive symptoms and suicidal ideation, particularly for those living in group homes (234). Adoption has similar affects: adoptees are at increased risk for suicide attempts compared to non-adoptees (235). The risk for suicide attempts and completed suicide significantly increases with increasing number of placements (i.e., changes in residence) (236). Research shows that Native youth in foster care are at risk, as well. Similar to parental boarding school attendance, Native youth who were taken from their parents reported significantly more sexual abuse and suicide attempts than those not taken from their parents $(49,105)$. Research also has shown that Native youth in the child welfare system were 3.7 times more likely to report suicidal ideation (across three time points) than their non-Native counterparts, even after controlling for living arrangement and number of placements (237). Several studies conducted among Native adults have shown that the effect of foster care placement lingers into adulthood. One study conducted among Metis adults (age 20-59 years) found a correlation between history of foster care experience and suicidal ideation, although boarding school attendance was not a significant correlate of ideation (117). A second study among Metis adults (age 25 and up) found a significant association between foster placement and both depression and suicidal ideation (238). Foster care placement also appears to be directly associated with alcohol dependence in American Indian women, but not American Indian men (134). It is possible that this difference may reflect gender-based coping $(38,39)$.

Removing children from their family has developmental consequences that may be detrimental to forming healthy relationships and cultural identity, the generational transmission of traditions and parenting skills, and mental health $(31,48,239,240)$. These practices undermined parents' rights and the ability of future generations to raise their children in traditional ways, disrupting community-level structures and social order that protected women and children. The physical, emotional, and sexual abuse rampant in Native lives were rare occurrences prior to colonization (241). The removals and abuses culminated in generations of collective silence and unresolved grief from feelings of stigma, shame, inadequacy, and guilt related to the abuses, including student-on-student abuse from identifying with the aggressor or being forced to do it $(242,243)$, and the boarding school experience in general $(31,203,244)$. The legacy of these historical events remains on the minds of many youth and adults $(200,245-$ 
247). Thinking of these losses has been linked with depression and substance use in youth and young adults $(178,246,248)$ and depressive symptoms in adults $(232)$.

\subsubsection{Parenting and Child-Rearing}

Boarding school attendees often are not prepared as parents to care for their children or to have healthy intimate relationships due to traumas during development that have impaired ability for attachment and familial transmission of traditional parenting skills. Being deprived of traditional child-rearing role models (249), many parents have modeled the abusive experiences of the boarding schools and foster homes $(31,229,240)$.

Traditional value systems, such as the relational worldview and extended kinship family structure, where parenting and family were at the core, were also disrupted by assimilation practices $(216,250-258)$. At present, in many cases, the kinship networks have been weakened and family structure changes frequently; fathers and extended family are not always present in their children's lives (257).

Several studies have described parenting among mothers who experienced childhood sexual abuse (259-261), including how trauma and depression impact parenting $(101,262)$. However, few studies assess how child sexual abuse victimization affects fathers. It is important to understand the generational consequences and mental health impact on all abuse victims. An AI-SUPERPFP study examined the association between childhood sexual abuse and parenting satisfaction and parenting role impairment (101). Although childhood sexual abuse did not have a significant effect on satisfaction, the researchers found a strong association between childhood sexual abuse and parenting impairment (OR = 3.56; $\mathrm{Cl}: 1.29,9.77)$, but for the Southwest tribal area only.

Suicidal behaviors have been linked to perceptions of ineffective parenting in youth of other racial/ethnic and Indigenous groups (263-265). For example, paternal overprotection was positively associated with suicide attempts in Indigenous Sami youth in Norway (263).This may be related to parent-child conflict from generational acculturation differences which appears to be a factor in suicidality among immigrant and Micronesian youth $(266,267)$. In Latinas, although parent-child conflict has been shown to be significantly and positively associated with suicide attempts, maintaining cultural values, such as familism, was negatively associated with parent-child conflict (267). For Asian immigrant youth, both maternal overprotection and parentchild conflict have been positively associated with depression, although maternal warmth was negatively associated with depression (268). Although empirical research on suicidality in American Indians has not examined the effects of parenting, the relation between ineffective 
parenting and youth suicidality has emerged as a theme in qualitative studies, especially in relation to physical, sexual, and emotional abuse $(26,240,269)$.

Traumatized parents may impart the consequences of their trauma to their own children. Literature documenting the long-term generational consequences of trauma among children whose parents survived the Jewish Holocaust is the most descriptive, encompassing more than 500 studies. Children of Holocaust survivors display problems coping with stress and a higher tendency for PTSD, especially those whose mothers were diagnosed with PTSD $(194,270)$. In addition, the second generation exhibits lower psychological well-being and poorer parenting compared to those with no Holocaust background (195). Moreover, if both parents are children of Holocaust survivors the negative effects may extend to third generation grandchildren.

Empirical research in Native populations is beginning to examine the generational effects of life stressors. Data from grandparents (of Native youth) who participated in the 1950 urban relocations show direct and indirect negative effects on their adult female children's mental health, substance use, and parenting ability which were, in turn, linked to poor mental health and delinquency in their grandchildren (271). Findings from the Semi-Structured Assessment for the Genetics of Alcoholism study in American Indians suggest that both assaultive trauma and PTSD symptoms were significantly, though moderately, heritable (272). This moderate heritability is consistent with general population findings $(273,274)$. Results from another study showed a positive association between parental boarding school attendance and depressive symptoms in their adult children. The offspring of these participants also reported significantly higher levels of childhood adversity, adult traumas, and perceived discrimination than offspring of non-attendees (275). The researchers speculate this may be related to stress proliferation or heightened sensitivity to perceived stressors.

\subsubsection{Potential Models}

Researchers have proposed several environmental and biological models to explain the generational effects of traumatic stress focusing on Holocaust survivors and their offspring. The most predominant include psychodynamic, sociocultural, family system, and biological models, as well as an integration of the four $(198,276)$. More recently, epigenetic models have been proposed (277). Psychodynamic models propose that children of survivors identify with and internalize, albeit unconsciously, their parent's trauma. Specific to survivors of the Jewish Holocaust, it is thought that the stress of repressed Holocaust experiences, unresolved grief, and social mistrust are internalized by their children who fail to emotionally detach during adulthood, in part through overprotective parenting. Similar to generational family violence 
theory (278), sociocultural models posit that a family's culture, social norms, and beliefs affect the trauma response via parenting behaviors that are modeled by their children (279). Family system models focus on parent-child enmeshment and communication style. This model proposes parent-child role reversals that may restrict child development. This model suggests that generational effects occur through non-verbal, vague, guilt-inducing communication and silence about Holocaust experiences, which is widespread in surviving families $(280,281)$. This is important in terms of American Indian populations, as well, because silence also is common among First Nations boarding school attendees (282). Biological models posit a genetic or biological predisposition to changes in the biological stress response due to trauma $(199,283$, 284). Although the above models provide some level of explanation, they may be inadequate, because meta-analysis both confirms and refutes previous results (199). Epigenetic models are promising to fill this gap, particularly because generational transmission of hypothalamicpituitary-adrenal (HPA) axis function has been demonstrated in animal studies $(285,286)$.

Epigenetic alterations are heritable, potentially reversible variations in phenotypic expression from context-dependent, gene-environment interactions $(286,287)$. Epigenetic mechanisms may partially explain how the effects of traumatic stressors are passed from parents to their offspring. Researchers speculate that an adverse pre-natal and early life environment, including ineffective parental care, can affect the epigenome and may have mental health implications (288-290). As evidence, animal models have shown increased methylation in rat pups raised by less-nurturing mothers and that upon maturity these pups are more sensitive to stressors, such as confinement that restricts their movement (291). Vulnerability to stress dysregulation may be transmitted from mother to child via epigenetic alterations that may be mediated by biological stress-response mechanisms, such as the HPA axis, a major endocrine system involved in coordinating short-term responses to stress (292). When this type of change occurs during a critical developmental window, it may serve to recalibrate biological systems that influence the response to subsequent traumatic exposure, because the HPA axis is influenced by early life experiences $(285,293-295)$. This may help explain the neurobiological susceptibility to stress in trauma survivors and subsequent generations. For example, low cortisol levels have been found in adult offspring of Holocaust survivors and in infants whose mothers were directly exposed to the trauma of the World Trade Center collapse during pregnancy (296-298). This is further supported by studies that show increased methylation in newborns exposed to maternal depression (299). The changes appear to be related to glucocorticoid programming in utero due to maternal childhood exposure to adversity, such as 
Holocaust survivors (297). Several studies in humans support an epigenetic model for suicide as well as depression and PTSD, noted suicide risk factors (6, 7, 295, 300-302).

Epigenetic mechanisms, a non-reductionist, more holistic approach, have been proposed to help explain what is referred to as the generational transmission of historical trauma in Native populations through modifications in the stress response, especially with regard to adverse childhood experiences (303); however, there are no empirical studies to date. This is a promising line of prevention research because animal studies have shown the potential for reversing DNA methylation in adult offspring through L-methionine supplementation (304). In Native communities, research of this type is complicated by distrust from a history of unethical research conducted in this population, most recently the sharing of blood samples without permission and not returning them as the university institutional review board (IRB) protocol required (210). This is especially troublesome because some tribes have cultural beliefs related to biological human material (305). Tribal self-governance established in the 1980s and 1990s through amendments to the Indian Self-Determination and Education Act enabled tribes to regulate research by establishing their own IRBs. At a minimum, proposed research must be collaborative and provide benefits that are meaningful and useful to the participants and tribal community members (306).

\subsection{Psychosocial Protective Factors}

Many Native communities have suicide prevention programs that stress the protective value of cultural traditions/spirituality and strengthening community relations; however, few have been evaluated for effectiveness (307). Increasing protective factors has been an effective population-level public health injury prevention approach; child safety seats, seat belts, and air bags have reduced motor vehicle-related deaths and barriers on the Golden Gate Bridge have reduced suicide deaths $(308,309)$; however, relatively few studies have examined factors protective against suicidal ideation and attempts. In this emerging area of research, results indicate that religion/spirituality (310-312), strong social support $(313,314)$, family cohesion (82, $92)$, and high self-esteem $(99,113,315)$ are protective against suicidal ideation and attempts.

\subsubsection{Spirituality}

Religion and spirituality may give meaning and purpose to life that is conducive to emotional well-being; they may provide comfort during life stress and may play a role in appraisal of adverse life experiences (316-318). Research shows that religion/spirituality are 
associated with better mental health in both youth and adults $(319,320)$. Religious community members may connect troubled youth to mental health care or serve as formal or informal mental health care providers (321). Regarding suicide deaths, religion may provide an avenue for social integration and social support (3, 322), particularly in women (323). For example, while African Americans report significantly more life stress than their White counterparts, suicidality is lower for African Americans than other racial/ethnic groups (311). African Americans also report significantly higher levels of religiosity and church attendance, which may account for the difference (324). One study showed a protective benefit of religion on suicidal ideation in African American adults even in the presence of discrimination and depressive symptoms (166). A study in college students found that spiritual well-being was protective against suicidal ideation, although religious participation was not (325). In American Indian adults, high cultural spiritual orientation acted as a buffer against lifetime suicide attempts; however, no significant association was found between commitment to either cultural spirituality or Christian beliefs (310).

\subsubsection{Social Support}

As a consequence of the colonization practices disrupting American Indian family structure and traditional parenting (31), the transmission of factors deemed protective against suicidality were disrupted as well, including language (326), cultural identity (113), and social support from extended kinship networks (94). Several studies have examined the protective role of social support, family cohesion, and self-esteem on suicidal ideation and attempts in Native youth, with varying results. A comparison of suicidal ideation in youth from three different tribal areas showed that social support was protective against ideation in Pueblo youth, and family cohesion was protective in Southwest youth, while neither appeared protective for ideation in Northern Plains youth (86). Research shows that family cohesion is protective against suicide attempts in boys and girls of all racial/ethnic groups (82, 92, 94, 327); however, differences were found in a study of American Indian youth living in an urban area (99). Although social support was protective against suicide attempts in youth raised in the urban area, it was not protective for reservation-reared youth now living in this urban area. A study of contemporary boarding school students found differences in the effects of social support from family and peers (328). Their results showed that low family support was positively correlated with ever attempting suicide and high peer support was correlated with higher current suicidal ideation. Because social support processes can be culturally determined $(329,330)$, it is important to use measures that reflect social support systems within a given cultural group (331). 


\subsubsection{Self-esteem}

Self-esteem levels vary by racial/ethnic group, with meta-analysis showing lower selfesteem in American Indians compared to White, Black, and Hispanic Americans, but not Asian Americans (332). The racial/ethnic differences in levels of self-esteem may be influenced by cultural values, as well as demographics. For example, high self-esteem was positively associated with individualism, which is contrary to American Indian culture, which is higher in collectivism (333). Despite this difference, research shows that higher levels of self-esteem appear protective against suicidal ideation across racial/ethnic groups $(267,315)$, including American Indian youth (113).

\subsubsection{Resilience}

Resilience, continuity (self and cultural), ethnic identity (personal and collective), and coping are influenced by psychosocial development and are embedded in sociocultural context. The concept of resilience denotes the ability to positively cope with and adapt to stress and adversity (334). This is an emerging area of research without a common underlying theoretical construct or standard operationalization in either general or Native populations $(112,335-338)$.

Components of the resilience construct, such as a positive attributional style and higher levels of agency, have been shown to buffer suicidality $(339,340)$. A recent review of this literature expressed the importance of using validated, multi-item inventories to measure resilience to address methodological concerns (339); however, only one study has assessed the psychometric properties of resilience measures in American Indian populations (341).

Studies in Native populations have examined resilience in relation to multi-level influences in youth $(336,342)$; as a moderator between violence and development of PTSD symptoms (343); its relation with self-esteem, enculturation and Native spirituality $(344,345)$; surviving a family suicide (346); associations with personal and social assets, such as pride, self-esteem, mastery, and parental and social support (347); and, as a framework for understanding youth suicide and domestic violence prevention research $(112,348)$.

Several mechanisms have been proposed for positively adapting after traumatic events (349), including: psychodynamic (secure attachment) (350), neurobiological (351), epigenetic (352), developmental (353), and social learning (279). However, the literature for trauma and resilience is not as elucidated as the trauma and stress research. 


\subsubsection{Continuity}

Having a secure sense of oneself (self-continuity) and one's culture (cultural continuity) as a connected part of the past, present, and future has been shown to be protective against suicide $(15,28,29)$. In turn, disruptions to personal or cultural persistence are suicide risk factors. Developmentally, critical transitions take place during adolescence that may disrupt a younger person's sense of self-continuity and their ability to cope with and adapt to life stressors. Research has found self-continuity practices (such as ability to see oneself moving into the future) in non-suicidal youth; however, minimal practices were noted in suicidal youth (15). This line of research also has noted cultural variations in perceptions of self-continuity, possibly reflecting a more analytic western, as opposed to a more holistic Native, worldview (354). Whereas Native youth viewed continuity through relational solutions, non-Native youth tended to choose concrete elements, such as name to judge their persistence through time $(355,356)$. This research also showed that for Indigenous people, suicide rates varied from place to place, but non-Indigenous suicide did not, indicating the presence of certain community elements for the tribal bands in this study that reported zero suicides ( $n=111 ; 56.6 \%$ ) over the 14-year time period of the study $(28,29)$.

The dramatic changes that have occurred in Indigenous communities have disrupted a sense of community, or cultural, continuity which has also affected the transmission of potential protective cultural elements to younger generations, such as language and cultural identity. However, the disruption has not been consistent across US tribes or Canadian First Nations bands. Research findings show remarkable differences in First Nations youth suicide rates across levels of cultural continuity markers (land claims, self-government, band-run services [i.e., education, police and fire, health, and child services], presence of a cultural facility, and women in government). Tribal bands with fewer cultural continuity markers had drastically higher youth suicide rates than those with more markers. Moreover, bands with all eight markers reported zero youth suicide deaths $(28,29)$. Also, bands with a conversational knowledge of their language had fewer youth suicides than bands without this knowledge, independent of other markers of cultural continuity (326). If Native youth suicide is related to disrupted development, it stands to reason that being involved in and identifying with one's culture may be a key protective factor. Culture provides a foundation that gives meaning to life and a way to see oneself moving into the future $(316,357)$. Culture also sets limits to behaviors through taboos, including taking one's own life (358). 


\subsubsection{Enculturation}

Culture consists of belief systems that provide people with ways of making sense of the world. Culture consists of "systems of socially constructed meanings, beliefs, and values that symbolically convey interpretations of what reality is and ought to be" (359). Culture is further described as "webs of significance" (360), as well as a "tradition of interpretation" (357). Whether due to assimilation policies, for survival, or as an adaptation, Native American culture has undergone vast changes (361). Responses to these changes have included revision of cultural practices; cultural revitalization; and, as often occurs when cultures meet, a syncretic blending of Native, non-Native, and inter-tribal practices $(361,362)$.

In the context of Native health, several researchers have argued the protective benefit of enculturation; however, results vary across studies. Zimmerman et al. defined enculturation as the "process by which individuals learn about and identify with their traditional ethnic culture" (363). In studies of American Indians, enculturation is frequently composed of three components: cultural identity, participation in traditional activities, and participation in spiritual activities. Using this conceptualization, buffering effects have been noted for alcohol abuse in adults $(178,364)$ and discrimination and depression in Navajo youth $(365,366)$. However, enculturation did not buffer the effect of discrimination in American Indian adults (178).

Some studies have examined individual components of enculturation, primarily cultural identity. For example, strong cultural identity in Hawaiian youth was a statistically significant risk factor for suicide attempts, while Hawaiian ethnicity itself was not (76). In another study, higher maternal American Indian cultural identity was significantly associated with higher socialemotional development in their toddlers (258). In addition, high levels of some aspects of ethnic identity (e.g., pride) appear to significantly suppress the impact of perceived discrimination on depression in First Nations adults (367). Consistent operationalization of ethnic identity and enculturation is an important consideration.

Results vary in studies of suicidal ideation and attempts as well. For example, enculturation appeared protective for suicidal ideation in a study of Midwest American Indian youth (113); however, a study that used cultural identity alone showed no significant association for either ideation or attempts in Southwest youth (99). In another study, no significant association was found between ideation and cultural identity in youth from three tribal areas (Southwest, Northern Plains, Pueblo) (86). It has been suggested that including language in the cultural identity construct may help eliminate or reduce discrepancies in the results (368), because language is strongly intertwined with culture. As previously noted, a conversational knowledge of traditional language acted as a buffer against suicide deaths (326), so we might 
expect similar results for suicidal ideation and attempts. In addition, strong ethnic identity was associated with a strong sense of community and positive affect in Northern Plains American Indian youth (369). This is important because connectedness to community has been shown as a statistically significant protective factor for ideation in Native adults (191).

Identity formation is an important developmental and psychosocial adolescent task that has implications for health and well-being in adulthood (370-373), including the emerging adulthood of most college students (374). Healthy development can buffer the negative effects of stressors like discrimination (365). Barriers to identity development in American Indian youth include exposure to and difficulty sorting out conflicting values from the dominant culture, limited number of adequate adult role models, limited parent-child communication, and trying to find their place in sometimes ethnically and tribally diverse sociocultural contexts (375). It is important to not only understand the parallel development of resilience, self-esteem, and identity, but also the developmental impact of traumatic stressors and coping skills.

\subsubsection{Coping}

Coping is defined as "constantly changing cognitive and behavioral efforts to manage specific external and/or internal demands that are appraised as taxing or exceeding the resources of the person" (376). Because ability to cope with stress is dependent on psychosocial development, it is more difficult for children to deal with adversity; they have not developed the coping strategies or accumulated the necessary resources to adapt to stressful situations. More successful strategies, like problem-focused coping, come with life experience (37). Culture also is an important factor in the stress-coping process, and may affect the types of stress experienced, their appraisal, and preferred and available coping strategies $(40,366)$. In addition, choice of coping strategy is often gender-based, for example, research shows that women tend to seek social support when under stress more often than men do $(38,39)$.

The way we respond, or adapt, to perceived life stressors (i.e., cope) may affect our health. Unhealthy responses (e.g., substance use, anger, denial, or venting) can be detrimental to health (377-379). Research shows that emotion-focused coping responses (escapism, selfblame, minimization) are positively associated with depressive symptoms; whereas problemfocused coping responses (instrumental action, negotiation) are negatively associated with depressive symptoms, especially in combination with high self-efficacy (380). A variety of coping classifications have been noted including: (1) active (primary) coping, such as problem solving or support seeking to help change the situation or one's emotional reaction; (2) accommodative (secondary) coping, such as distraction or acceptance to adapt to the situation; and, (3) 
disengagement strategies such as avoidance or withdrawal (381). Coping can also be anticipatory in that strategies such as vigilance are used to prevent or minimize future potential adversity, for example, in relation to anticipated discrimination (382).

Several studies have examined stress, coping, and various health outcomes among Indigenous people. A study among the Yupik found healthier coping strategies and greater wellbeing in residents of a more traditional Yupik village who reported using religion/spirituality more, and alcohol/drugs less, than residents of a non-traditional village (383). Focus groups conducted among Canadian First Nations provided broader explanations. For example, Iwasaki and colleagues examined coping strategies for life stressors in First Nations diabetics (384). Coping themes included (1) focusing on strengths (individual, collective); (2) maintaining a balance of mind, body, and spirit; and, (3) promoting positive transformations through spirituality, interdependence, and cultural identity.

For many Natives, coping, resilience, traditional worldview, and healing are intertwined. Research conducted among Canadian First Nations residential school survivors revealed similar culturally informed ways of coping and healing from physical and sexual abuses they experienced in the schools (385). Healing strategies include maintaining or reestablishing: (1) a balance in life; (2) interconnectedness to the world; (3) traditional spirituality, healing practices, and cultural values; (4) connections to family and community; (5) a connection with nature; and (6) guidance from tribal role models or mentors. Also, preliminary data from American Indian youth in the study area found that they used a variety of unhealthy coping strategies, such as cutting, substance use, and violence to cope with stress in their lives (26). The youth also emphasized the importance of keeping busy, engaging in outdoor activities, seeking family and community support, and involvement in cultural activities (e.g., hand games, singing, participating in ceremonies) to deal with stress in a constructive way. These findings show that cultural values and traditions are promising coping strategies to help American Indian communities deal with past and current life stressors.

Although several studies have examined associations between stress and suicide, a limited number have examined how coping affects suicidality and related risk factors. Suicidal youth and adults tend to use fewer and less effective coping strategies than their equally stressed counterparts (34). For example, youth who had not attempted or thought about attempting suicide reported significantly more cognitive reframing and talking about their feelings than youth reporting suicidal behaviors (34). A study of multi-racial youth showed that problem-focused coping was positively associated with suicidal ideation (35). A study in Navajo youth found that primary (e.g., problem-solving) and secondary (e.g., acceptance) coping were 
negatively associated with depressive symptoms; disengagement (e.g., denial) was positively associated with depressive symptoms (381). A study of coping strategies and substance use found that American Indian youth reported using significantly more denial and self-blame and significantly less active coping and acceptance than White youth (386).

\subsection{Addressing the Gaps}

In summary, this dissertation addresses several gaps and inconsistencies evident in the literature. First, this research will expand knowledge of risk factors for suicidal ideation and attempts in American Indian youth in an under-researched tribal area. Second, this research will broaden the field by examining the association between suicide risk and culturally unique risk factors, such as childhood adversity, discrimination, generational out-of-home placement, unresolved grief, and ineffective parenting in American Indian tribal college students. Perceptions of ineffective parenting have been linked to suicidality in other Indigenous groups (263). In addition, research in the general population has shown a significant association between complicated, unresolved grief and suicidality (387). This research will be the first to examine these associations in American Indians. Third, this work will add to the literature by examining the association between suicidality and potential protective factors, such as resilience, enculturation, and cultural values, as well as the effect of coping skills and social support on suicidality in American Indian tribal college students. In addition to filling gaps, this research is innovative in several ways. Although conceptualized $(24,25,36)$, a stress-coping model has not been tested in this population. The setting is also unique in that limited research has been undertaken in Montana-Wyoming tribal areas. In addition, limited suicide research has been conducted with American Indian college students and no suicide research has been conducted with students attending tribal colleges $(88,388)$. This is a unique opportunity because seven (19\%) of the nation's 36 accredited tribal colleges are located in these states. The tribal college setting offers a unique opportunity to test this model, which allows consideration of the effect unique cultural strengths have on culturally unique, as well as generalized stressors, in the lives of American Indians. Suicidality is a major public health issue in this population. A comprehensive understanding of culturally unique life stress and cultural buffers will help to inform effective interventions and reduce morbidity and mortality related to this problem. 
The following specific aims will be used to fill these gaps:

Specific Aim 1: Identify gender-specific individual-level risk factors associated with suicidal ideation/attempts for American Indian and White Montana high school students using pooled random sample secondary Youth Risk Behavior Surveillance System (YRBS) data.

Hypothesis \#1. American Indian youth will have a higher prevalence of suicidal ideation/attempts than White youth.

Hypothesis \#2. Sadness/hopelessness will show the strongest positive association with suicidal ideation/attempts for all youth.

Hypothesis \#3. There will be differences in suicidal ideation/attempt risk factor profiles for the American Indian and White youth (e.g., types of victimization and coping strategies).

Specific Aim 2: Identify gender-specific individual-level risk factors associated with suicidal ideation and suicide attempts in American Indian students attending Montana reservation and urban high schools using pooled volunteer sample secondary YRBS data.

Hypothesis \#1. Reservation youth will have a lower prevalence of suicidal ideation and suicide attempts than the urban youth due to access to protective influence of cultural practices and ethnic identity development.

Hypothesis \#2. There will be differences in risk factor profiles (e.g., types of victimization and coping strategies) for suicidal ideation and attempt for the reservation and urban settings for girls and boys.

Specific Aim 3: Test a model of the relation between life stressors (i.e., adverse childhood experiences, discrimination, generational out-of-home placement, unresolved grief, and ineffective parenting skills), poor mental health, enculturation, resilience, coping skills, social support, self-esteem, and gratitude on suicide risk in American Indian tribal college students using primary survey data. 
Hypothesis \#1. Higher levels of life stressors (i.e., adverse childhood experiences, discrimination, generational out-of-home placement, unresolved grief, and ineffective parenting skills) and poor mental health will be positively associated with suicide risk.

Hypothesis \#2. Higher levels of enculturation, resilience, constructive coping skills, social support, self-esteem, and gratitude will mitigate suicide risk. 


\section{CHAPTER 2. A COMPARISON OF RISK FACTORS ASSOCIATED WITH SUICIDAL IDEATIONIATTEMPTS IN AMERICAN INDIAN AND WHITE YOUTH IN MONTANA}

\subsection{Abstract}

Introduction. We examined racial/ethnic and gender-specific associations between suicidal ideation/attempt and risky behaviors, sadness/hopelessness, and victimization in Montana American Indian and White youth with aggregated 1999-2011 Youth Risk Behavior Survey data.

Methods. We used logistic regression to calculate odds ratios and $95 \%$ confidence intervals in stratified racial/ethnic-gender groups.

Results. The primary results of this study show that although the American Indian youth had more statistically significant suicidal thoughts and attempts than the White youth, American Indian youth had fewer statistically significant risk factors compared to the White youth. Sadness/hopelessness was the strongest, and the only positively and statistically significant, risk factor for suicidal ideation/attempts common across all four groups. The unhealthy weight control cluster was a positive and statistically significant risk factor for the White youth and the American Indian girls; the alcohol/tobacco/marijuana cluster was a positive and statistically significant risk factor for the American Indian boys only.

Conclusion. Results show important differences across the groups and indicate directions for future research targeting prevention and intervention. Although there were differences in magnitude across the race/ethnic-gender groups, after sadness, victimization appears to be the most potent indicator of suicidal ideation/attempts in all groups. Mental health, school safety, dating violence, and substance use screening, and referral to culturally sensitive and genderspecific counseling are warranted. 


\subsection{Introduction}

Suicide deaths, non-fatal suicide attempts, and suicidal ideation (e.g., thinking about, considering, planning for suicide) are a worldwide public health problem for Indigenous (e.g., American Indian, Alaska Native, Canadian First Nation, New Zealand Maori, Australian Aborigine) people, especially those under age 25 years $(52,53,62,389)$. In the US, American Indian/Alaska Native (Al/AN) youth report significantly more suicide-related thoughts and behaviors than youth from other racial/ethnic groups, including attempting suicide up to 2.5 times more often than these other youth (78). As with other racial/ethnic groups, Al/AN girls report attempting suicide almost twice as often as Al/AN boys (82).

Poor mental health, especially depression, is consistently associated with the greatest risk for suicidal ideation/attempts $(11,120,121)$; however, there are other potentially modifiable factors. Research also shows a direct relation between suicidal ideation/attempts and psychosocial risk factors such as exposure to suicide by family or friend $(82,99)$, substance use $(99,113,120,390,391)$, and victimization, such as physical and sexual abuse $(53,82,102)$, dating violence $(97,104)$, and sexual assault $(49,97)$ across racial/ethnic groups. All in all, the prevalence of most of the risk factors is highest in AI/AN youth (78).

Studies have also shown associations between suicidal ideation/attempts and several other psychosocial risk factors, such as early risk-taking behaviors (264), issues around weight control and body image (390, 392), risky sexual behaviors (390), and bullying (393); however, these studies did not include Al/AN youth. Besides the potential risk factors listed above, other unique areas of concern for AI/AN youth include earlier initiation and more frequent use of alcohol, tobacco, and other illicit drugs (78), higher exposure to suicidal thoughts and behaviors (394), discrimination $(113,178)$, out-of-home placement (226), and family history of boarding school attendance (229).

Although suicidal ideation/attempts is a growing health concern among Al/AN youth, a limited number of studies have included AI/AN youth in the examination of racial/ethnic differences. Rutman et al. (80) examined differences between AI/AN and White youth in urban schools and Pavkov et.al. examined national level racial/ethnic differences (78); however, these analyses were largely descriptive and did not examine risk factor associations. Another study surprisingly found American Indian race/ethnicity to be a protective factor for 3 month suicidal ideation/attempts (11); however, this study was set in a region that differs demographically from western states that have multiple reservations and different tribes (www.census.gov; 2010 data). In addition, the researchers did not stratify by racial/ethnic group or gender (11). A recent 
study found both differences and similarities in risk factors for suicide attempts between Native and non-Native youth; however, they controlled for, rather than stratified by, gender (87).

The present study focuses on the state of Montana, which has the highest suicide rate in the 48 contiguous states. Montana has multiple tribal areas, including seven reservations and five urban areas. American Indians are the most visible minority group in Montana, comprising about $7.7 \%$ (76,000) (www.census.gov; 2010 data) of the total population. The purpose of the present analyses is to examine racial/ethnic and gender-specific associations between high-risk behaviors, sadness/hopelessness, victimization, and risk for suicidal ideation/attempts in American Indian and White youth in Montana using Youth Risk Behavior Survey (YRBS) data. To our knowledge, an analysis of YRBS suicide data for a Northern Plains state such as Montana has not been published. Understanding unique and shared gender- and racial/ethnicspecific risk factors associated with suicidal ideation/attempts is important to help inform suicide prevention intervention to decrease the prevalence of suicidal ideation/attempts in this vulnerable group.

\subsection{Methods}

\subsubsection{Sample and Participants}

The YRBS is a self-report epidemiological survey conducted in odd years in randomly selected high schools across the nation. The survey monitors health behaviors in six categories: unintentional injury and violence; tobacco use; alcohol and other drugs; sexual behaviors; physical activity; and dietary behaviors. A three-stage cluster sample design is used to produce a nationally representative sample of high school students (grades 9-12). The first-stage sampling frame is on the county level, the second is on the school level, and the third is on the classroom level. All students in the classroom level sample are eligible to participate. Schools, classes, and students may refuse to participate and are not replaced. Parental permission to complete the survey is secured locally following local parental permission procedures. The selfadministered in-person questionnaire was completed during one class period with responses recorded by the student on an answer sheet (395).

The school response rate for the annual sample of Montana schools $(N=50)$ ranged from $94 \%$ to $98 \%$ over the study time period. There are two tribally run schools in the state and they participate. The vast majority of schools are rural. For the present analyses, data for Montana American Indian (self-identified) girls $(n=628)$ and boys $(n=695)$ and White girls $(n=$ 
8814) and boys $(n=8715)$ in grades 9-12 were extracted from the YRBS for the seven combined odd years from 1999-2011 $(N=21,610)$.

\subsubsection{Variables}

Because changes had been made to the YRBS over the study time-period, we only included variables that were used in all seven years of data in the analyses; the questions were identical. The dependent variable was a positive response to at least one of the following: "During the past 12 months, how many times did you actually attempt suicide?" (yes = 1 or more times; no = 0 times), "During the past 12 months, did you ever seriously consider attempting suicide?" (yes; no), or "During the past 12 months, did you ever make a plan about how you would attempt suicide?" (yes; no). We determined seriousness of intent by the question "If you attempted suicide during the past 12 months, did any of your attempts result in an injury, poisoning, or overdose that had to be treated by a doctor or nurse?" (yes; no). Preliminary analyses showed little to no difference in the statistical significance of individual risk factors for suicidal ideation and suicide attempts. Therefore, to increase power, we used cluster analysis to combine the variables into more meaningful clusters and to help to reduce multicollinearity in variables that were similar. The variables were coded in the direction of high risk. In addition to sadness/hopelessness (feeling sad or hopeless every day for 2 weeks or more during the past 12 months), we selected 29 independent variables and grouped them into 9 clusters: (1) alcohol, tobacco, and marijuana use (current and binge drinking, riding with someone who had been drinking and driving while drinking during past 30 days, current tobacco use, current marijuana use); (2) unhealthy weight control (fasting, taking diet pills, purging to lose weight during the past 30 days); (3) early risk-taking behavior (smoking cigarettes, drinking alcohol, using marijuana, or having sex before age 13); (4) partner victimization (past 12 months dating violence, ever sexually assaulted); (5) lack of personal safety at school (feeling unsafe [past 30 days], being threatened [past 12 months]); (6) risky sexual behaviors (more than 4 sex partners in lifetime, more than 1 sex partner in the past 3 months, alcohol/drug use before last sexual intercourse, no condom use with last sexual intercourse); and (7) weapon carrying (carried a weapon and/or a gun during the past 30 days); as well as two illicit drug clusters (8) injection (ever use heroin, steroids, and needle drugs) and (9) inhalant [cocaine (ever, current) and ever use methamphetamines]). Because the clusters contain different numbers of risk behaviors, we used the mean for each cluster in the analyses. 


\subsubsection{Procedures}

Variable clustering is a widely accepted statistical method used to reduce the dimensionality of prediction models (396). However, there are not a sufficient number of studies in the literature concerning grouping these specific variables to form a well-grounded hypothesis of which variables to cluster. As we did not want to bias the analyses by improperly clustering variables, we allowed the data to show us where the clusters were. Similarity in some items would also have created problems with multicollinearity. We used the non-hierarchical version of Proc Varclus in SAS Version 9.2 to create the clusters. It uses an iterative process to create groups of variables that are as correlated as possible within a cluster and as uncorrelated as possible with variables in other clusters (396). We used partitive clustering with the default 0.7 eigenvalue threshold to split the clusters. We selected 9 clusters at the point where the clusters were formed of variables that were not substantially different from other iterations.

\subsubsection{Statistical Analyses}

Analyses were stratified by racial/ethnic group and gender and were carried out with SAS Version 9.2. First, simple frequencies were established to determine gender and racial/ethnic-specific prevalence estimates. Chi-square tests of association were used to determine significant racial/ethnic and gender between-group differences. A Bonferroni correction was used to account for multiple testing, so differences in prevalence estimates were considered statistically significant at the $p<.0005$ level. We then conducted logistic regression analyses (forward selection) using age as a control variable. We hypothesized that depression might be the key underlying risk factor, because it consistently shows the greatest independent risk for suicidal ideation/attempts $(11,121)$. Depression is also associated with several of the risk factors discussed here (e.g., bullying, smoking, and alcohol, marijuana and other substance use) (123). Because sadness/hopelessness was the only reasonable surrogate we had for depression in the YRBS, it served as a proxy in the logistic regression models. We also hypothesized that American Indian youth will have a higher prevalence of suicidal ideation/ attempts than White youth and that there will be differences in suicidal ideation/attempt risk factor profiles (e.g., types of victimization and coping strategies) for American Indian and White youth. Proc SurveyLogistic was used to account for complex sampling and to facilitate generalizability across all Montana high school students. All analyses were weighted to account for the complex sampling design and to reduce nonresponse bias (397). 
Following their code of regulations, all aspects of this study, including this article, received approval from the Rocky Mountain Tribal Institutional Review Board located in Billings, Montana.

\subsection{Results}

\subsubsection{Descriptive Statistics}

Descriptive statistics can be seen in Appendix A; Table 1. During the study time-period, more than twice as many American Indian youth $(n=169 ; 15.5 \%)$ in Montana reported attempting suicide one or more times during the past 12 months than White youth $(n=1111$; $7.1 \%)$. In addition, twice as many American Indian youth $(n=54 ; 4.9 \%)$ reported an injury associated with attempted suicide, in comparison to White youth ( $n=361 ; 2.3 \%)$. Both attempt ( $n=106 ; 19.6 \%)$ and attempt with injury $(n=35 ; 6.5 \%)$ were highest in the American Indian girls and a higher proportion of these girls reported considering $(n=165 ; 26.8 \%)$ and planning for $(n=131 ; 21.0 \%)$ an attempt during the past year.

Almost twice as many girls reported sadness/hopelessness as boys in their respective racial/ethnic-groups, with American Indian girls ( $n=267 ; 43.2 \%)$ reporting the highest. Compared to the White girls, the American Indian girls reported significantly more suicidal thoughts $(p=.0003)$, suicide attempts $(p<.0001)$, injury with attempt $(p<.0001)$, sadness/hopelessness $(p<.0001)$, early risk-taking behaviors $(p<.0001)$, feeling unsafe at school $(p<.0001)$, dating violence $(p<.0001)$, and fasting to lose weight $(p<.0001)$. Compared to the White boys, a significantly higher percentage of American Indian boys reported suicidal thoughts $(p<.0001)$, suicide attempts $(p<.0001)$, sadness/hopelessness $(p<$ .0001), smoking cigarettes or marijuana or having sex before age $13(p<.0001)$, sexual assault $(p<.0001)$, and using diet pills, fasting, or purging to lose weight $(p<.0001)$.

\subsubsection{Logistic Regression Analyses}

Results from the regression analyses (Appendix A; Table 2) show differences and similarities across the racial/ethnic and gender groups. Unless otherwise indicated, all statistically significant risk factors are positively associated with suicidal ideation/attempts. As hypothesized, sadness/hopelessness was one of the strongest risk factors for suicidal ideation/attempts and was the only statistically significant risk factor common across all four groups: American Indian girls (OR = 4.13 [2.51, 6.81]); American Indian boys (OR = 6.23 [3.59, 10.80]); White girls $(\mathrm{OR}=6.57[5.76,7.49])$; White boys $(\mathrm{OR}=8.80[7.53,10.28])$. The 
unhealthy weight control cluster was a statistically significant risk factor for suicidal ideation/attempts for the White boys and girls and the American Indian girls, but not the American Indian boys. In addition to sadness/hopelessness, unhealthy weight control was also the only significant risk factor for the American Indian girls. There was an inverse association between age and suicidal ideation/attempt in all groups except the American Indian boys. For the American Indian boys, in addition to sadness/hopelessness, the alcohol/tobacco/marijuana cluster and the weapon carrying cluster were statistically significant risk factors for suicidal ideation/attempts.

The White girls had more significant behavioral risk factors for suicidal ideation/attempts than any other group. In addition to sadness/hopelessness and the unhealthy weight control cluster, other statistically significant risk factors for suicidal ideation/attempts in these girls included alcohol/tobacco/marijuana, early risk-taking behaviors, lack of personal safety at school, partner victimization, and weapon carrying. Like White girls, White boys also showed significance for lack of personal safety at school, early risk taking behaviors, and partner victimization. White boys were the only group where risky sexual behaviors were also a significant risk factor.

\subsection{Discussion}

The primary results of this study show that although the American Indian youth had more statistically significant suicidal thoughts and attempts than the White youth, they had fewer statistically significant risk factors compared to the White youth. Specifically, while the American Indian youth reported significantly more partner victimization, lack of personal safety at school (feeling unsafe at school only), early risk taking behaviors, and risky sexual behaviors than the White youth, these clusters were significant risk factors for suicidal ideation/attempts for the White youth only.

Sadness/hopelessness was one of the strongest risk factors for suicidal ideation/ attempts and the only risk factor that was common across the four racial/ethnic-gender groups. The importance of sadness/hopelessness as a risk factor for suicidal ideation/attempts is not surprising because we know that depression is the strongest risk factors for suicidal behaviors (11, 120-122). Our data show that American Indian youth have significantly more sadness/hopelessness than their White counterparts. This finding is consistent with some studies $(123)$, but not all $(78,398)$. The reason for this discrepancy in findings on depression in American Indian youth is unclear; however, it may result from variation in the age range of the 
study populations. For example, some studies focus on middle school youth (398), who report the lowest prevalence of depressive symptoms, while others focus on high school $(78,79)$ aged youth or combine the age groups $(81,99,123)$. This is important since research shows that depressive symptoms increase with age (399).

The fact that substance abuse is greater in the American Indian youth may be an indication that they are self-medicating. Substance abuse and depression frequently co-occur in youth (400), so examination of factors underlying these co-morbidities in this population is warranted. That three out of the four groups had issues with unhealthy weight control is not surprising when seen from the perspective of researchers who see fasting, purging, or taking diet pills to lose weight as a method of coping with stress, similar to the use of substances as a maladaptive coping strategy (401).

The limited number of statistically significant risk factors for the American Indian youth may indicate that the YRBS does not capture the areas of greatest risk for them. For example, a risk factor that was not included was discrimination, which has been linked to substance use and suicidal ideation in middle school aged American Indian youth $(113,177)$.

Also, baseline data from a Canadian First Nations longitudinal health survey show significantly greater suicidal ideation/attempts among both youth and adults who had at least one parent or grandparent attend the now renowned abusive federal boarding schools for American Indian children $(206,209,212)$ compared to offspring of non-attendees (229). In addition, several generations of American Indian children have been disproportionately placed in foster care or adopted out, almost exclusively with non-Native families $(208,222)$. The abuse and lack of nurturance in the federal boarding schools where children were not even allowed to speak their own language was a poor preparation for learning to parent their own children (31). Even removing children from their families has consequences that may be detrimental to forming healthy relationships and cultural identity, (e. g., the intergenerational transmission of traditions), as well as mental health (resulting in substance abuse to cope with stress) $(31,48$, 239, 240).

It is interesting that partner victimization was not associated with suicidal ideation/ attempts in the American Indian youth in the multiple variable models even though it was significantly more prevalent in the American Indian than White youth and was also a significant risk factor in the White youth. This is not consistent with the limited sexual assault data in Native youth, where ever being sexually assaulted was a significant risk factor for suicide attempts (49, 82). Due to the cross-sectional nature of the data in the current study and the wording of the sexual assault question, it was not possible to determine the context in which the violence 
occurred, i.e., whether child sexual abuse was involved. So we ran post hoc multiple variable models for the American Indian youth with dating violence and sexual assault as separate, unclustered variables to see if they contributed differently to the model, but they did not. Neither dating violence nor sexual assault were statistically significant risk factors for suicidal ideation/ attempts in these post hoc analyses.

Most studies of suicide in American Indians focus on risk and relatively few studies have examined protective factors. Research indicates that several psychosocial factors, such as religion/spirituality $(310,311,324)$ and strong social support (82) may be protective against suicidal ideation/attempts across racial/ethnic groups. Enculturation (i.e., participation in traditional social and spiritual activities and cultural identity) and high self-esteem have been reported to be protective against suicidal ideation among middle school aged American Indian youth but this has not been studied in older youth (113). Since factors related to self-esteem and culture are not available in our dataset we could not test this hypothesis in our sample.

This study is subject to several limitations. First, these data are limited by the nature of self-report data, namely, underreporting and recall bias; and, by the fact that they are crosssectional, which makes attribution of causality difficult. Second, data are only collected on youth enrolled in school. Youth who have dropped out of school and may be at even higher risk are not included. The average dropout rate for Montana American Indian students during the study time-period was about $6.5 \%$, compared to about $2.5 \%$ for White students (402). In addition, the YRBS no longer includes socioeconomic variables, which may be important given the extensive poverty of American Indian youth $(11,403)$. There are also no questions related to other risk factors which may be relevant for American Indian youth, such as discrimination $(113,178)$, exposure to suicide of others $(82,99)$, out-of-home placement $(404)$, or access to and use of mental health care services (405). In addition, there is only one question that could possibly reflect depression and no questions related to PTSD or other relevant mental health issues (11). To test whether low power could be responsible for the lack of significant risk factors in the American Indian youth, we used JMP Pro Version 11 to calculate effect sizes. Using Cohen's effect size taxonomy (406), these analyses revealed that our gender-specific sample sizes for the American Indian youth correspond to $80 \%$ power for effect sizes around .20-.22, which is equivalent to small or perhaps medium effect sizes. This leads us to believe that low power is not an issue.

It should also be noted that the YRBS does not differentiate between what are perceived as negative behaviors/emotions and aspects of these behaviors/emotions that may have culturally distinct meanings in American Indian youth. For example, the concept of depression or 
sadness/hopelessness, though normally seen as negative in other populations, can have positive connotations for American Indian youth, i.e., be perceived as a strength, if it is born with a sense of honor and kept in one's heart as a reminder to have compassion for others (407). Another example is the topic of tobacco smoking, normally perceived as a risk behavior in mainstream society, but which may be part of traditional ceremonial practices to which the youth would respond affirmatively. An American Indian youth may also admit to carrying a weapon. However, the survey does not differentiate between a weapon that is part of ceremonial and/or dance regalia, i.e., as symbolic of warrior status, so we do not know how they interpret 'weapon' when they respond. Furthermore, the YRBS does not differentiate between urban and reservation American Indian youth. As stated above, it also does not include questions related to protective factors. Inclusion of these types of variables in future research may contribute to improving knowledge of the issues underlying suicidal ideation/attempts in this population. In spite of these limitations, the YRBS is a readily available source of risk factor data that covers a broad range of suicidal ideation/attempts questions that show both convergent and discriminant validity (408).

As strengths, this study combined seven years of YRBS data for statistically meaningful numbers of American Indian youth and no study to date has examined gender- and racial/ethnic-specific differences in this regionally representative sample. Many American Indians are cautious, meaning that openness with outsiders is minimal and information is not freely shared (409). However, given the anonymous nature of the YRBS, these results are more likely to provide an accurate reflection of American Indian youth behavior than structured interviews. In addition, the complex sampling design and non-response weighting allow for generalizability across Montana high school students as well as students from states with similar population characteristics.

Despite the public health importance of suicide, only limited research has been conducted on suicide risk in American Indian youth (389). Despite the caveats, this study has some important public health implications. It shows that factors predicating suicidal ideation/ attempts in American Indian and White youth differ and that our current databases are probably not capturing some of the risk factors that are relevant for prediction of suicidal ideation/ attempts in American Indian youth. The fact that age was inversely associated with suicidal ideation/attempts indicates that early intervention should be a high priority. The current data also tell us that screening for sadness/hopelessness, school safety, and substance use, as well as referral to culturally meaningful and gender-specific counseling are important. Future research aimed at identifying risk factors for suicide in American Indian youth should include 
culturally relevant factors such as discrimination and out-of-home placement, as well as protective factors such as social support and enculturation in order to design evidence-based interventions based on cultural strengths. 


\section{CHAPTER 3. RESERVATION-URBAN COMPARISON OF SUICIDAL IDEATION AND ATTEMPTS IN AMERICAN INDIAN YOUTH}

\subsection{Abstract}

Introduction. Although research is beginning to address suicide risk in urban American Indian youth, most suicide research in this population focuses on American Indian youth living on or near reservations. Relatively few studies have made comparisons between urban and reservation populations. The objective of this study was to identify gender and location-specific individual-level risk factors associated with suicidal ideation and suicide attempts in American Indian youth.

Methods. Biennial data for 6417 American Indian high school students were extracted from the Montana volunteer sample Youth Risk Behavior Survey data for pooled years 2003 to 2011. Logistic regression was used to identify gender-specific individual-level risk factors associated with suicidal ideation and attempts in American Indian students attending Montana reservation and urban high schools.

Results. Findings show that the prevalence of suicidal ideation and suicide attempts did not significantly differ between reservation and urban American Indian youth. However, regression analyses showed differences and similarities in significant risk factors for ideation and attempts for girls and boys across the locations. Fewer differences were noted for girls than boys. Sadness/hopelessness was the only statistically significant risk factor for suicidal ideation and attempts common for girls and boys in both settings. Some form of violence (partner victimization, lack of school safety) was positively and significantly associated with either ideation and/or attempts in almost all groups. Partner victimization was significantly associated with both outcomes for girls, but was inconsistent for boys. Lack of school safety was significantly associated with suicide attempts across the four groups, but was not a significant risk factor for ideation. Unlike girls, reservation and urban boys differed in identified risk factors for both ideation and attempts. Reservation boys who contemplated but did not attempt suicide showed significant associations with early risk-taking behaviors, partner victimization, and weapon carrying. Urban boys who actually attempted suicide had far more significant risk factors than reservation boys. 
Conclusion. The results indicate that although the prevalence of suicidal ideation or attempts did not differ for American Indian youth in reservation and urban settings, the risk factors did, especially for boys. In girls, risk factors differed by location primarily for ideation. This is important since American Indian youth report significantly more suicide-related thoughts and behaviors than other youth. Future research that focuses on multilevel and culturally unique risk and protective factors is essential to design meaningful prevention and intervention programs. 


\subsection{Introduction}

Research consistently shows higher suicidality (deaths, non-fatal attempts, and ideation) in Indigenous populations worldwide, compared with their national counterparts $(51,52)$. Data also show clear tribal and geographic variations in the prevalence of suicidal ideation and attempts and associated risk factors in American Indian youth (76, 410, 411). Although studies are beginning to address suicide risk in urban American Indian youth $(49,80,112)$, most suicide research in this population focuses on American Indian youth living on or near reservations (62, $82,113,114,120,124)$. Relatively few studies have made comparisons between urban and reservation populations $(28,99)$. Of these, Chandler and Lalonde found higher suicide rates among British Columbia First Nations youth living in urban and surrounding areas as opposed to rural or remote locations (28). In addition, a study of 314 American Indian youth noted higher suicidal ideation and psychosocial problems in reservation-reared youth than urban-reared youth; there were also differences in types of risk factors for suicide attempts in these youth (99). In that study, depression showed a significant association with suicide attempts for the reservation-reared youth only and alcohol abuse/dependence showed a significant association for the urban-reared youth only. Neither study examined gender differences.

Chapter 2 of this dissertation reports differences in risk factors for American Indian and White youth, but did not have data to identify school location (412). That study showed that sadness/hopelessness was the strongest, and the only statistically significant, risk factor for suicidal ideation/attempt common for the American Indian and White youth. In that study, although the American Indian youth had a significantly higher prevalence of suicidal thoughts and attempts than the White youth, they had fewer statistically significant risk factors. Since living in a socioeconomically and culturally homogeneous subculture on a reservation is different than living in a more diverse urban environment, it is not unlikely that psychosocial risk factors and types of coping mechanisms may differ between reservation and urban youth. Our goal with the current study was to investigate potential differences. For example, reservation youth have more ready access to cultural traditions and support from extended family that may confer protective benefits on suicide risk $(94,113)$, while American Indian youth who live in urban areas are in an environment where they are in the minority, where stressors such as discrimination may increase suicide risk (113).

Our hypothesis was that cultural differences (e.g., diminished social support from extended family and limited access to protective influences of cultural practices and ethnic identity development) (413) would put urban residing youth at risk for lower sense of belonging 
(191) that would in turn place them more at risk for either suicidal ideation or suicide attempts than the reservation youth. Based on research showing that boys and girls differ developmentally with boys consistently taking more risks than girls (414) and that coping strategies are often gender-based $(38,39)$, we also hypothesized gender differences in risk behaviors and psychosocial factors that are associated with suicidal ideation and attempts.

\subsection{Methods}

\subsubsection{Participants}

Participants were a volunteer sample of American Indian high school students from Montana reservation $(n=3748)$ and urban $(n=2669)$ schools who agreed to take the Youth Risk Behavior Survey (YRBS) to provide an overview of risk factors for American Indian youth. Montana is unique in that the state Office of Public Instruction invites all schools with a large population of American Indian students to participate in the volunteer sample of the YRBS administration. This allows for a comparison of American Indian students in schools that are located (1) wholly or partially within or that share a border with Montana reservations and (2) in more "urban" areas (i.e., off-reservation schools situated entirely outside reservations). Many of these off-reservation schools are located in areas with populations ranging from 75,000 160,000 .

Across the US overall, about $60 \%$ of American Indians live in urban areas. Montana differs in that a higher percentage of American Indians live on reservations (60\%); while about $40 \%$ live in urban areas $(415)$. About $17 \%(N=30)$ of the 178 Montana school districts are located on or near a reservation. Of these, about $70 \%(N=21)$ participated in the volunteer sample YRBS during each year of the study time-period (2003-2011). Schools on or near a reservation participated at about the same proportion as the more urban schools $(N=104)$. There were about $40 \%$ more participants from reservation than urban schools. About $9 \%$ of the urban youth attended alternative schools for those more at-risk of dropping out (416). For the present cross-sectional survey analyses, data for American Indian (self-identified) reservation girls $(n=1860)$ and boys $(n=1888)$ and American Indian urban girls $(n=1284)$ and boys $(n=$ 1385 ) in grades 9-12 were extracted from this unique YRBS sample for the five combined odd years from 2003-2011 $(N=6417)$. 


\subsubsection{Outcome Measures}

Two dependent variables were used in the analyses: suicidal ideation and suicide attempts. Suicidal ideation consisted of a positive response to at least one of the following: "During the past 12 months, did you ever seriously consider attempting suicide?" (yes; no) or "During the past 12 months, did you ever make a plan about how you would attempt suicide?" (yes; no) and a negative response to attempting suicide. Suicide attempts consisted of a positive response to "During the past 12 months, how many times did you actually attempt suicide?" (yes = 1 or more times; no $=0$ times). Cases were removed from the analyses when no response was provided for suicidal ideation $(n=32 ; 0.5 \%)$ or suicide attempts $(n=116$; $1.8 \%)$.

\subsubsection{Risk Factor Cluster Determination}

Because many of the individual risk factors in the YRBS are similar in content and we did not want to bias the analyses by subjectively grouping them, we did cluster analyses to combine them into more meaningful risk factor clusters, as well as to reduce model complexity and the risk of multicollinearity. Variable clustering is a widely accepted statistical method used to reduce the dimensionality of prediction models by grouping similar items. The cluster analyses were done with the non-hierarchical version of Proc Varclus using partitive clustering with the default 0.7 eigenvalue threshold to split the clusters (396). It uses an iterative process to create non-overlapping groups of variables that are correlated within a cluster and uncorrelated with variables in other clusters. Because changes had been made to the YRBS over the study time-period, we only included variables that were used during all five years of data collection. Variables of interest remained unchanged during the study period. Nine clusters were selected: (1) alcohol, tobacco, and marijuana use (during past 30 days: current and binge drinking, riding with someone who had been drinking, driving while drinking, tobacco use, marijuana use); (2) inhalant drug use (cocaine [ever, past 30 days], methamphetamines [ever]); (3) injection drug use (ever use: heroin, steroids, needle drugs); (4) early risk-taking behavior (smoking cigarettes, drinking alcohol, using marijuana, having sex by age 12 or under); (5) lack of school safety (feeling unsafe [past 30 days], or threatened [past 12 months]) at school; (6) partner victimization (past 12 months dating violence, ever sexually assaulted); (7) risky sexual behaviors (more than 4 sex partners [lifetime], more than 1 sex partner [past 3 months], alcohol/drug use and/or no condom use before last sexual intercourse); (8) unhealthy weight control (past 30 days: fasting, taking diet pills, purging to lose weight); and, (9) weapon carrying (past 30 days: carried a weapon and/or a gun). Because each cluster contains a varying 
number of risk factors, the mean for each cluster was used in the analyses to scale for consistency. We also examined sadness/hopelessness (feeling sad or hopeless every day for 2 weeks or more during the past 12 months), the only mental health variable available in the dataset. This served as our proxy for depression, a well-known suicide risk factor $(11,121)$. The variables were coded in the direction of high risk. Less than $1.5 \%$ of responses were missing for each of the independent variable clusters, so imputation was not necessary and these cases were treated as missing.

\subsubsection{Statistical Analyses}

Analyses of suicidal ideation and suicide attempts were stratified by school location (reservation or urban) as well as by gender, because girls think about and attempt suicide more often than boys $(82,92)$. Simple frequencies were calculated to determine reservation/urban and gender-specific prevalence estimates of risk factor clusters. These were calculated as the proportion of the total responses that were affirmative. Chi-square tests of association were conducted to determine significant reservation/urban between-group differences in boys and girls. To account for multiple comparisons, Bonferroni corrections were used so differences in the estimates were considered statistically significant if they reached the $p<.004$ level or lower. Logistic regression analyses (forward selection) were calculated for suicidal ideation and suicide attempts separately. Odds ratios (with $95 \% \mathrm{Cl}$ ) were adjusted for age and all other model risk factors.

Following their code of regulations, all aspects of the project received approval from the Rocky Mountain Tribal Institutional Review Board located in Billings, Montana.

\subsection{Results}

\subsubsection{Descriptive Statistics}

There were no significant differences in the prevalence of suicidal ideation or attempts by location for girls or boys. However, the reservation girls reported significantly less sadness/hopelessness $(40.4 \% ; p<.0001)$, injection drug use $(3.2 \% ; p<.0001)$, partner victimization $(13.4 \% ; p<.0001)$, and weapon carrying $(6.1 \% ; p<.0001)$ than the urban girls. In contrast, they reported more alcohol/tobacco/marijuana use $(41.9 \% ; p<.0001)$ compared to the urban girls. Like the reservation girls, reservation boys were also less likely than the urban boys to carry weapons $(22.1 \% ; p<.0001)$ or inject drugs $(3.9 \% ; p=.0004)$. However, they were 
more likely than urban boys to report alcohol/tobacco/marijuana use $(39.1 \% ; p=.0030)$ and risky sexual behaviors $(31.1 \% ; p<.0001)$ (Appendix B; Table 1).

\subsubsection{Risk Factors for Suicidal Ideation in Girls}

Appendix B; Table 2 shows that for both reservation and urban girls, sadness/ hopelessness and partner victimization were statistically significant risk factors associated with suicidal ideation. However, in reservation but not urban girls, unhealthy weight control $(\mathrm{OR}=$ $1.80[1.07,3.02])$ was also a risk factor for ideation. Inhalant drug use $(\mathrm{OR}=0.73[0.54,0.98])$ showed an inverse relation with ideation that was found in reservation girls only.

\subsubsection{Risk Factors for Suicidal Ideation in Boys}

Sadness/hopelessness was the strongest risk factor for ideation for both boys and girls. Sadness/hopelessness was positively and significantly associated with ideation in reservation $(\mathrm{OR}=3.83[2.78,5.27])$ and urban $(\mathrm{OR}=5.34[3.77,7.56])$ boys (Appendix B; Table 3). But again, reservation boys had more significant risk factors associated with suicidal ideation than the urban boys. Although reservation boys were less inclined to carry weapons than urban boys, for those who did, weapon carrying $(O R=1.19[1.03,1.37])$ was associated significantly with suicidal ideation. Weapon carrying was not associated with suicidal ideation in urban boys. Early risk-taking behaviors $(\mathrm{OR}=1.74[1.02,2.99])$ and partner victimization $(\mathrm{OR}=2.22[1.30$, 3.78]) were also significantly associated with suicidal ideation in reservation but not urban boys.

\subsubsection{Risk Factors for Suicide Attempts in Girls}

The models show that the profiles of reservation and urban girls were very similar with respect to suicide attempts (Appendix B; Table 4). The majority of the risk clusters were positively and statistically significant correlates for suicide attempts in girls regardless of location. In addition to sadness/hopelessness, common statistically significant risk factors for suicide attempts for these girls included partner victimization and lack of school safety, as well as unhealthy weight control, early risk-taking, and weapon carrying. The only notable difference by location was that inhalant drug use $(\mathrm{OR}=1.39[1.07,1.82])$ was significantly associated with suicide attempts in urban girls only.

\subsubsection{Risk Factors for Suicide Attempts in Boys}

In contrast to the girls, urban and reservation boys showed clearly different profiles for suicide attempts (Appendix B; Table 5). In reservation boys, only two risk factors were 
significantly associated with suicide attempts: sadness/hopelessness (OR = 2.75 [2.02, 3.74]) and lack of school safety $(O R=1.26[1.05,1.51])$. In urban boys, on the other hand, in addition to sadness/hopelessness $(\mathrm{OR}=2.03[1.44,2.85])$ and lack of school safety $(\mathrm{OR}=1.20$ [1.01, 1.42]), unhealthy weight control (OR = $3.05[1.53,5.92])$, early risk-taking behavior $(\mathrm{OR}=1.96$ $[1.14,3.36])$, and partner victimization $(O R=1.81$ [1.06, 3.10]) were significantly associated with attempts. Thus, risk factors associated with suicide attempts in urban boys were similar to those in girls.

\subsection{Discussion}

We found no significant difference in the prevalence of suicidal ideation or attempts by reservation-urban location for girls or boys. This is in contrast to previous research where reservation youth reported a higher prevalence of ideation and attempts than urban youth (99). Consistent with our hypotheses, the study findings show variation across gender profiles in risk behaviors and psychosocial factors associated with suicidal ideation and attempts, as well as differences across the reservation-urban settings. A finding of interest is that reservation girls and boys reported less sadness/hopelessness than their urban counterparts, which partially supports our original hypothesis that urban youth would be more isolated. The question is whether social support or other cohesive cultural factors contribute to a smaller prevalence of sadness/hopelessness in reservation youth. Because sadness/hopelessness was significantly associated with both ideation and attempts in these data, this finding indicates that further research in this area is warranted.

Although the youth reported using substances, only inhalant drug use was significantly associated with a study outcome (suicide attempts) and that was for urban girls only. However, it is puzzling that inhalant drug use showed an inverse relation with ideation for reservation girls. We found no significant difference in inhalant use between reservation and urban location, but previous research in this population found significantly higher levels of inhalant drug use in American Indian girls and boys, including methamphetamines, compared to their White counterparts (412). This is consistent with the literature $(80,417)$. Methamphetamine use is an important public health problem with wide-ranging implications for tribal communities (418-422). More research is needed to understand its link with suicidal behaviors. Overall, substances may serve as a way for youth to cope with stress or violence in their lives.

Reservation girls also were less likely to carry a weapon or to have experienced partner victimization than urban girls. Partner victimization was a significant risk factor for both suicidal 
ideation and attempts in reservation and urban girls but was inconsistent for boys. From our previous research, we know that significantly more American Indian than White youth report partner victimization and data in the current study show that it was significantly more prevalent among urban than reservation girls (412). Although in the current study we do not know the circumstances surrounding the reported sexual assaults (age of occurrence; perpetrator), research often shows a higher prevalence of child sexual abuse in American Indian youth compared to White youth (423). Regarding the higher prevalence in urban girls, research shows that American Indian girls are highly vulnerable to being lured into sex trafficking in urban settings and are actually targeted for commercial sexual exploitation (424). In addition, American Indian women are 2.5 times more likely to experience sexual violence than women in general with most of reported cases ( $86 \%$ ) being perpetrated by non-Native men (425), which may explain why urban girls are even more at-risk than reservation girls.

It is interesting that there was an association between lack of school safety and suicide attempts, but not suicidal ideation, across the youth groups. This may be one indicator of the cumulative effect of multiple stressors on attempting, as research has shown a significant association between the number of risk factors an individual accumulates and their risk for suicide attempts (87). Except for reservation boys, the number of significant risk factors increased from ideation to attempt.

Some form of violence (partner victimization, lack of school safety) was positively and significantly associated with either ideation and/or attempts in almost all groups; coping strategies such as substance use and unhealthy weight control were more indicative of suicide attempts than ideation only. This sheds some light on the progression from ideation to attempts in American Indian youth. Research is beginning to examine this distinction (426-428), but further research is needed to understand which life stressors and/or coping strategies predict the progression from ideation to attempts in American Indian youth.

One study strength was our ability to distinguish between suicidal ideation and attempt. We operationalized ideation as those who only thought or planned an attempt rather than including those who also went on to attempt, because most people who think about attempting suicide do not go on to actually make an attempt (429). This has implications for understanding the progression from thinking about to actually attempting suicide. Such an understanding could better inform prevention and intervention in this vulnerable population. Also, few studies have examined risk factors for suicidal behaviors in both reservation and urban settings and to the best of our knowledge none have included both suicidal ideation and attempts. This large 
database allowed us to compare risk factors related to both suicidal ideation and suicide attempts in American Indian youth in both reservation and urban settings.

A major limitation is that the data did not allow us to investigate the effect of socioeconomic, structural, family, or cultural factors. This may be the reason for the lack of significant risk factors for attempts in reservation boys. American Indians face high rates of poverty, unemployment, and low educational attainment which have been linked to many poor health outcomes. While poverty has been shown to have an independent association with youth suicidality $(11,116)$, social fragmentation may play a larger role than poverty $(118,119)$. Also, many colonization practices disrupted American Indian family structure (31), inhibiting the transmission of factors deemed protective, including language and cultural identity $(113,326)$, as well as social support from extended kinship networks (94). Important risk factors such as discrimination or out-of-home placement were not included $(113,229)$. In addition, youth may be reluctant to accurately respond to sensitive topics such as suicidal ideation and attempts or sexual assault; however, the YRBS is anonymous and the percent responding affirmatively to these questions indicates that the likelihood of this bias is low. Other limitations include recall bias related to self-report and collecting data only on youth enrolled in school when data from dropouts could be relevant. In addition, since this is a convenience sample the findings may not fully reflect the populations under study. Also, although many Montana cities meet the Census definition of an urban area, the results may not be generalizable to states with larger metropolitan statistical areas (www.census.gov). Access to health care is also an issue, especially mental health care. Research shows an association between discrimination and unmet mental health care needs in American Indians (171). Although the distribution of Indian Health Service funding allocated for mental health and substance abuse services $(7 \%)$ is comparable to the health spending in the US overall, per capita expenditures are much less (430, 431).

In conclusion, American Indian youth suicide is costly in terms of the loss of young lives and hope for the future, as well as the disruption it causes families and communities and the related economic costs (432). The results indicate that although the prevalence of suicidal ideation or attempts did not differ for American Indian youth in reservation and urban settings, the risk factors did. Early intervention and prevention that is culturally responsive and meaningful to tribal youth, families, and communities is imperative (433). Future research that focuses on multilevel risk and protective factors is essential for our ability to design meaningful prevention and intervention programs. 


\section{CHAPTER 4. TESTING A STRESS-COPING MODEL OF SUICIDE RISK IN A TRIBAL COLLEGE POPULATION}

\subsection{Abstract}

Introduction. American Indian youth and young adults have the highest rates of suicide deaths, non-fatal attempts, and ideation in the US. Available data for American Indian college students show higher suicidal ideation than college students overall. Suicide-related data for tribal college students are not available. The objective of this study was to investigate general and culturallyunique psychosocial risk and protective factors associated with suicide risk in this vulnerable group.

Methods. Through on-line and paper self-administered surveys, we collected information in 2012-2013 from 187 American Indians who were tribal college students in Montana and Wyoming. With these data, we tested a stress-coping model (situated within a cultural-historical context) to determine associations between suicide risk and culturally unique life stress, coping skills, enculturation, cultural values, resilience, and social support. We used logistic regression to calculate odds ratios and $95 \%$ confidence intervals.

Results. Study results showed low levels of past year suicide risk, especially past year suicide attempts. Statistically significant (positive) stressors of lifetime suicide risk were adverse childhood experiences, other traumatic events, parenting difficulties, discrimination, and thinking of historical losses. Gratitude, social support, participant boarding school attendance, and higher current income mitigated suicide risk.

Conclusion. Although past 12-month suicidal ideation was similar to American Indian high school students in the same tribal region, past 12-month attempts were remarkably low. The results show important areas for future research, including an in-depth understanding of a potential protective influence of the tribal college environment for suicide attempts. The results also have implications for the development of socioeconomic, structural, family- and culturebased interventions and lend support for the importance of widely used Native-developed interventions, such as American Indian Life Skills and Healthy Relationships. 


\subsection{Introduction}

American Indian youth and young adults have the highest rates of suicide deaths, nonfatal attempts, and ideation in the US $(52,62,63)$. Available data for American Indian college students show higher suicidal ideation than college students overall (388). Distinct differences in risk factors among American Indian college students, as well as potential protective factors within this group, demand further attention in addressing suicidality as a major public health threat. Limited research on suicide has been conducted with American Indian college students and, to our knowledge, no suicide research has been conducted with students attending tribal colleges $(88,388)$. The tribal college setting offers a unique opportunity to test the effect cultural strengths have on culturally unique, as well as generalized stressors, in the lives of American Indian students. A comprehensive understanding of culturally unique life stressors, cultural buffers, and ways of coping will help to inform effective interventions and reduce suicide-related morbidity and mortality in American Indians.

A stress-coping model of Native health has not been tested; however, a model has been conceptualized $(24,25,36)$. Using primary cross-sectional survey data, we tested a stresscoping model (Appendix C; Figure 1) of suicide risk to determine associations between suicide risk and culturally unique life stress, coping skills, enculturation, cultural values, resilience, and social support in American Indian tribal college students in Montana and Wyoming. The model is situated within a cultural-historical context (30-33). The model represents culturally unique and generalized stress-coping and focuses on cultural strengths and values that may serve as stress buffering mechanisms. Limited protective factor research with Native populations shows the importance of focusing on the protective influence of cultural strengths and values, as opposed to a deficit, risk factor based focus $(21,44)$.

Although depression consistently shows the strongest association with suicide risk (11, 120-123), there are other, potentially modifiable, psychosocial factors for American Indians related to suicide risk, including culturally unique life stressors such as adverse childhood experiences, discrimination, generational out-of-home placement, historical losses, unresolved grief, and parenting difficulties. In the general population, research has shown associations between suicide risk and psychosocial risk factors, including both past and current life stressors $(31,113,125,138,208,222,229,434)$. Although many risk factors are similar across racial/ ethnic groups, higher prevalence estimates are found in American Indian youth and adults (78, 90). This is especially true for childhood adversity and traumatic experiences overall, where 
significantly higher estimates have been reported by American Indians compared to the general US population (138).

Perceived discrimination, a current life stressor, has been linked to suicide risk in several populations, including American Indian youth $(113,165,435)$, as well as young adults in other minority groups (164). In terms of past stressors, research also shows significantly greater suicide risk among both youth and adults who had at least one parent or grandparent attend boarding school compared to non-attendee offspring (229). In addition, several generations of American Indian children have been disproportionately placed in foster care or adopted out, almost exclusively with non-Native families $(208,222)$. This practice continued until relatively recently, despite evidence which shows that removing children from their family has detrimental consequences in forming attachments and cultural identity by interrupting the generational transmission of traditions, values, and parenting skills $(31,48)$. Out-of-home placement also has been linked to suicide risk in other populations $(234,235)$. When considering the impact of these and other past traumas, one must also take into account the effects of complicated, unresolved grief, which showed a significant association with suicide risk in a largely white, widowed population (387). In addition, thinking of these and other historical losses has been linked with suicide risk factors in Native youth and adults (178, 232, 246, 248).

Despite many risks, there are potential protective factors for suicidality, such as strong social support (99), high levels of religious commitment/spirituality $(166,310,325)$, high selfesteem $(113,267,315)$, and constructive coping $(35,381,383,436)$. In addition, research has shown enculturation to be protective for suicidal ideation in American Indian youth (113), including a conversational knowledge of traditional language (326). Strong cultural identity may play a role in developing and maintaining a well-defined concept of self-identity. This is important because maintaining a strong and persistent sense of self through developmental transitions also appears protective against youth suicide (437). Nonetheless, research on the relation between suicide risk and culturally unique stressors and protective factors in the most at-risk age group of American Indians, college students, is non-existent, necessitating further investigation through the application of a culturally tailored stress-coping model.

Based on this culturally informed stress-coping model, the first hypothesis is that higher levels of life stressors (i.e., adverse childhood experiences, discrimination, generational out-ofhome placement, unresolved grief, ineffective parenting skills) and poor mental health will be positively associated with suicide risk. The second hypothesis is that higher levels of enculturation, resilience, constructive coping skills, social support, self-esteem, and gratitude will mitigate suicide risk. 


\subsection{Methods}

In line with community-based participatory research principles, the project team consisted of tribal members from the Planting Seeds of Hope (PSOH) youth suicide prevention project based at the Rocky Mountain Tribal Leaders Council (RMTLC). All procedures were established in collaboration with the local PSOH project team. Following their code of regulations, this project received approval from the Rocky Mountain Tribal Institutional Review Board (RMTIRB) located in Billings, Montana. According to the RMTIRB code of regulations, the study data are the property of the participating tribes under the protection of the RMTLC. To protect confidentiality, no names, tribal affiliations, or tribal colleges have been identified. The study results are in aggregate form, that is, not tribal college- or reservation-specific. Where applicable, the project also was approved by reservation- or tribal college-based IRBs; the remaining participating tribal colleges provided letters of support to attest to their approval. The project also was reviewed and approved by the West Virginia University IRB.

\subsubsection{Participants and Population}

Participants were a volunteer sample of American Indian (self-identified) tribal college students from Montana and Wyoming and were recruited by $\mathrm{PSOH}$ research team members, in cooperation with administrators at each individual school. Each school was provided with a tailored recruitment flyer (Appendix C). Inclusion criteria included Montana-Wyoming tribal college students ages 18 years and older who self-identified as American Indian.

A majority of the American Indian college students in these states attend tribal colleges (63\%) compared to $7 \%$ nationwide. Additionally, seven (19\%) of the nation's 36 accredited tribal colleges are located in this area (221), serving approximately 2700 American Indian students during the study time-period (Fall 2012; www.nces.ed.gov). Nationwide, about half of the tribal college students are over the age of 24 years and approximately $42 \%$ are men. The tribal colleges are all located on reservations and most only offer two year degrees.

\subsubsection{Procedures}

The survey (Appendix C) consisted of established epidemiologic measures (details are below) complemented with additional questions informed by focus group data collected in the same tribal area (March-August 2012) by the Primary Investigator (PI) and PSOH staff (26), as well as through extensive consultation with PSOH and other RMTLC staff. Online survey software (www.qualtrics.com) was used to develop the web-based survey. Access to data is 
protected by password and meets federal Health Insurance Portability and Accountability Act standards. The survey was pilot tested with four American Indian college students from a Montana university. At the request of administration at some of the tribal colleges, a paper survey was added to the online version. Some schools elected to use the on-line or paper survey only; some chose to use both versions.

To ensure participant concerns related to taking the survey were met, participants who reported symptoms of depression, anxiety, posttraumatic stress disorder (PTSD), or suicidal thoughts or behaviors were encouraged to talk to a tribal society member or tribal college counselor. The tribal society members and college counselors were available before, during, and up to two weeks after taking the survey. The tribal society members were given an honorarium for their services.

\subsubsection{Incentives and Sharing of Results}

Upon completion of the survey, participants were given the option of having their name included in an incentive drawing. If they responded yes, the on-line survey participants were sent to a second (unlinked) survey to provide their contact information. Participants who completed the paper survey and wanted to be in the incentive drawing provided their contact information before leaving the classroom or computer lab. The original proposal allowed for a drawing for one $\$ 150$ item per school. However, some schools chose to have two winners of $\$ 75$ gift cards, which were purchased locally. Funds remaining at the end of the grant period were used to provide additional gift cards for participants.

Participants were also given the option to provide contact information at the end of the survey in order to receive study results, if desired. All contact information was stored separately from the data to ensure confidentiality. A tribal IRB-approved summary of aggregate results has been provided to participants and tribal stakeholders. In addition, de-identified datasets were provided to participating tribal colleges, where requested. This project was funded through the Center for Mental Health Services (CMHS) and the Substance Abuse and Mental Health Services Administration (SAMHSA) (Award \#SM57380-01) and through pre-doctoral dissertation funding through the Ruth Landes Memorial Research Fund (Award \#1005521R).

\subsubsection{Consent Process}

Although actual documentation of consent is not possible with on-line surveys, participants were not able to start the survey without attesting that they had read and 
understood the study description and that they agreed to participate. A standard in-person consent process was used for participants who opted for the paper survey.

\subsubsection{Data Collection}

Data collection took place during the fall 2012 through fall 2013 semesters. Although 376 students started the survey, the number of completed surveys was 338 , with the difference due to internet access issues, loss of interest, lack of information on American Indian identity, and non-Native or non-tribal college students completing the survey. In addition, an error was made in the skip pattern for the ever attempt suicide question with the on-line survey. Participants who answered yes to the ever attempt suicide question were taken to the next set of survey questions; participants who answered no to the ever attempt suicide question were taken to the end of the survey. The skip pattern error was rectified; however, the error had affected 151 surveys thereby lowering the current analytic sample to 187 participants. In addition, important measures were missing from the participant data affected by the skip pattern error, including mental health, lifetime traumatic events, and gratitude. Chi-square tests were run and there were no significant differences in demographic characteristics between the cases that were and were not affected by the skip pattern error, therefore, the analytic sample is still representative of the sample as a whole. Of the 187 participants in the analytic sample, 37.4\% $(n=70)$ completed on-line surveys and 62.6\% $(n=117)$ completed paper surveys. Chi-square tests found no significant difference in key demographics between the on-line and paper cases. Response rates ranged from approximately $3 \%$ to $22 \%$ across the participating tribal colleges. Student population for semester of participation was obtained from school administrators.

\subsubsection{Measures}

Total scores were positively associated with the name of the scale, for example, a high score on the stress scale equaled high stress; a high score on the social support scale equaled high support. Where possible, measures were selected that had been validated in American Indian populations.

\subsubsection{1. utcome Measure}

Suicide risk was measured with an 11-item dichotomized version of the Suicidal Ideation Questionnaire that had been culturally adapted for the American Indian Services, Utilization, Psychiatric Epidemiology, Risk and Protective Factors Project (AI-SUPERPFP) project (121, $137,438)$. A similar dichotomized scale showed good convergent validity with commonly used 
suicidality measures using a Likert scale format (439). Suicide risk scores ranged from 0 to 11 $(M=2.12, S D=2.51$, Cronbach's $\alpha=.82)$.

\subsubsection{Demographics}

Demographic covariates used in the analyses included commonly used and culturally relevant control variables: gender, age group, current annual household income, mother's education, and childhood home. We controlled for age and gender, because coping strategies are often age- and gender-based (37-39). Culture also is an important factor in the stresscoping process and may affect the types of stress experienced, their appraisal, and preferred and available coping strategies (40). Childhood home (whether raised on or off the reservation) served as a proxy for exposure to cultural influences.

\subsubsection{Generational Out-of-Home Placement Index}

The generational out-of-home placement index was assessed by a sum of Yes responses for two generations of boarding school attendance (parents, grandparents) and three generations of foster care/adoption (participant, parents, grandparents). Because participant boarding school attendance showed a significant inverse association with suicide risk in bivariate models, it was entered into the multiple variable models separately rather than as part of the generational summed index. The maximum possible score, based on total number of generational out-of-home placements, was 13 with a minimum baseline score of zero for no placements.

\subsubsection{Adverse Childhood Experiences (ACE)}

The childhood adversity measure was an adaptation of the Adverse Childhood Experiences study measure (131), using results from previously conducted focus groups (26). Six dimensions of childhood adversity (emotional abuse, physical abuse, sexual abuse, emotional neglect, physical neglect, childhood household dysfunction) were examined for a sum of Yes responses for a cumulative ACE exposure score ranging from 0-16 $(M=5.09, S D=3.46$, Cronbach's $\alpha=.80$ ). Emotional and physical abuse ${ }^{\star}$ included a Yes response to whether caregiver (mother/female; father/male) was: (1) verbally abusive and/or (2) physically violent or abusive to participant. Emotional neglect ${ }^{\star}$ was determined by a Yes response to: (1) not having

\footnotetext{
*Likert scales responses recoded to never experiencing the adversity $=0$ and any level of experience $=1$.
} 
emotional needs met and/or (2) participant feeling like they were a parent to their parent. Sexual abuse included a Yes response to: (1) ever being raped or being forced to have sex and/or (2) ever being touched or made to touch someone else in a sexual way by force. Physical neglect ${ }^{\star}$ included three questions regarding not having enough food to eat, enough clothes, or a decent place to live when growing up. Childhood household dysfunction included four queries (Yes, No) regarding childhood situations: lived with someone who had a problem with drugs or alcohol or a mental problem; parents divorced or separated; and, mother was treated violently.

\subsubsection{Traumatic Events Inventory}

In addition to the ACE score, a summed score for a traumatic events inventory (not including ACE) also was used with scores ranging from 0-24 $(M=5.73, S D=4.11$, Cronbach's $\alpha=.82$ ). This list was adapted from the Al-SUPERPFP study traumatic events list using input from the local project team (9): assaultive violence including interpersonal events (being raped or molested, physical abuse by a spouse or someone else, being robbed or mugged); noninterpersonal events (being in a disaster, a life-threatening accident, direct combat experience, witnessing violence); someone close to participant in a life threatening situation, raped or sexually abused, committed suicide, murdered, or died in a car crash; loss of mother or father; and other (open-ended) traumatic events.

\subsubsection{Parenting}

The parental control subscale from the Measure of Parenting Style Scale was used to assess perceived parenting skills and included questions related to the caregiver being overprotective, overcontrolling, critical, or making participant feel guilty during their first 16 years (440). To avoid multicollinearity, we elected to only use the control subscale, since the other subscales (indifference, abuse) overlapped with the ACE measures. Because there were more missing data with the father/male caregiver responses, we used the mother/female caregiver score only. Response options ranged from not true at all to extremely true. The mother's parental control subscale scores ranged from 5 to $20(M=12.77, S D=2.86$, Cronbach's $\alpha=$ $.57)$.

*Likert scales responses recoded to never experiencing the adversity $=0$ and any level of experience $=1$. 


\subsubsection{Discrimination}

The Everyday Discrimination measure was used to assess the occurrence and frequency of respondents' perception of unfair treatment from routine and relatively minor dayto-day interpersonal experiences (441). Response options for each question ranged from 1 to 6 , with 1 indicating almost every day and 6 indicating never. The summed reverse-coded responses were used to create the everyday discrimination score with scores ranging from 9 to $54(M=23.54, S D=10.97$, Cronbach's $\alpha=.91)$.

\subsubsection{Perceived Stress}

Perceived stress was measured with Cohen's 10-item Perceived Stress Scale (PSS) (442). The 5-point scale ranged from never to very often with scores ranging from 10 to $50(M=$ 28.27, $S D=6.51$, Cronbach's $\alpha=.82$ ).

\subsubsection{Historical Loss}

Historical loss perceptions were assessed with a 12-item measure asking how often the participant thought about (never to several times a day) a series of historical losses (200). Scores ranged from 12 to $72(M=35.94, S D=14.44$, Cronbach's $\alpha=.94)$.

\subsubsection{Depression}

Depressive symptoms were measured using the 20-item Center for Epidemiologic Studies Depression Scale (CESD) (443-446). Responses ranged from 1 (rarely or none of the time) to 4 (most or all of the time) for a scale range of 20 to $80(M=36.48, S D=10.54$, Cronbach's $\alpha=.89)$.

\subsubsection{Anxiety}

Generalized anxiety symptoms (past 6 months) were measured with the 7-item Generalized Anxiety Disorder (GAD-7) scale. Responses ranged from not at all to nearly every day for a scale range of 7 to $28(M=12.01, S D=5.27$, Cronbach's $\alpha=.94)(447)$.

\subsubsection{Posttraumatic Stress Disorder (PTSD)}

PTSD symptoms were measured with the 8-item PTSD-8 scale. Responses ranged from not at all to most of the time for a scale range of 8 to $32(M=15.12, S D=7.11$, Cronbach's $\alpha=$ .94) (448). 


\subsubsection{Grief}

Grief was measured using the UCLA Grief Screening Scale, an 11-item self-report inventory of past month grief symptoms with responses ranging from not at all to most of the time (387). Scale scores were summed with scores ranging from 12 to $60(M=32.54, S D=$ 11.60, Cronbach's $\alpha=.92$ ).

\subsubsection{Social Support}

Social support was assessed with Sarason's 6-item Social Support Questionnaire (449). Participants were asked to check all those that they could count on for help or support regarding being dependable, helping you relax, accepting you, caring about you, helping you feel better, and consoling you. The options, developed in consultation with the $\mathrm{PSOH}$ project team, included family members and other relatives, friends, clergy, spiritual elders, God/Spiritual Being, other, and no one. Scores were summed from 0 to 9 for each item for a scale range of 0 to $54(M=15.65, S D=10.40$, Cronbach's $\alpha=.96)$. Missing responses and no one were coded as 0 ; each checked option was coded as 1 .

\subsubsection{Self-esteem}

Self-esteem was measured with the 6-item Rosenberg Self-Esteem Scale validated by the Voices of Indian Teens Project (450). Responses ranged from strongly agree to strongly disagree. Scale scores ranged from 6-24 ( $M=19.23$; SD = 3.29; Cronbach's $\alpha=.81)$.

\subsubsection{Spirituality}

Spirituality was assessed using three-items from the AI-SUPERPFP study that asked about importance of (extremely important to not at all important), time spent on (every day or almost every day to not at all), and how often one finds comfort or guidance through religious or spiritual practices (never to very often) (310). Scores ranged from 3 to $15(M=10.92, S D=$ 3.28, Cronbach's $\alpha=.86)$.

\subsubsection{Resilience}

Resilience was measured using Wagnild's 14-item Resilience Scale (RS-14) (451, 452). Responses ranged from strongly disagree to strongly agree. The RS-14 scores range from 14 to $98(M=80.43, S D=16.41$, Cronbach's $\alpha=.97)$. 


\subsubsection{Gratitude}

Gratitude was measured with the 6-item Gratitude Questionnaire (range $=6-42 ; M=$ $34.87, S D=6.61$, Cronbach's alpha $=.76)(453)$. Responses ranged from strongly disagree to strongly agree.

\subsubsection{American Indian Cultural Identity}

Cultural identity was used as a measure of enculturation using the American Indian subscale of the 8-item Bi-Ethnic Identity Scale $(368,454)$. Responses ranged from not at all to very much or from not at all important to extremely important. Scores ranged from 8 to $40(M=$ 24.83, $S D=7.53$, Cronbach's $\alpha=.89$ ).

\subsubsection{Coping}

Coping was measured with the Brief COPE, a well-established abridged version of the COPE Inventory (455). The 28-item (14 subscale) Brief COPE measures various constructive and non-constructive coping dimensions. All 14 subscales, or a select number of subscales, can be used. In addition, variable reduction techniques, such as principal component analyses (PCA), have been used to reduce the dimensionality of the subscales (456). In the current study, constraining the PCA to retain two factors reduced the dimensionality of the 14 subscales to constructive and non-constructive coping for a more parsimonious model. Scores ranged from 16 to 64 and 12 to 48 , respectively, for the constructive $(M=44.59, S D=9.59$, Cronbach's $\alpha=.88)$ and non-constructive $(M=23.43, S D=6.18$, Cronbach's $\alpha=.80)$ coping factors. Responses ranged from I don't do this at all to I do this a lot.

\subsubsection{Participant Reaction to Participating}

Because of the sensitive nature of the survey and the history of research abuses in this population (210), three questions were included at the end of the survey to assess participant reactions to participating, including: feeling upset, thought worth the time, and felt free to skip questions or stop at any time (457). Responses ranged from not at all to a great deal.

\subsubsection{Statistical Analyses}

Analyses were conducted with SAS Version 9.3. First, simple frequencies were calculated for suicide risk and socio-demographic variables. Then, means, standard deviations, and Cronbach's alphas were calculated for each individual scale. Next, bivariate regression 
models were used to calculate odds ratios and 95\% confidence intervals using a suicide risk score proportion (suicide risk score/11) as the outcome variable.

Given the multitude and complexity of potential factors that may increase or mitigate suicide risk as compared with the limited sample size, steps were taken to determine the most parsimonious models for examining the impact of the independent variables on the overall suicide risk score, including: (1) running multicollinearity diagnostics to check for redundancy in the independent variables, (2) conducting subscale-level principal component analyses (varimax rotation) to reduce the dimensionality of the Brief COPE subscales, and (3) running a model using the three mental health scales to determine which one was most strongly and significantly associated with suicide risk for inclusion. Then, multiple variable logistic regression models were run using the suicide risk score proportion as the dependent variable. Non-statistically significant factors were removed for a more parsimonious final model.

The tolerance scores for the selected independent variables in these models were 0.46 or higher, so multicollinearity was not a concern (458). Depression was selected as the mental health scale to include in the model, because the strength of association between depressive symptoms and suicidality was the strongest of the three mental health scales (depression $(\mathrm{OR}=$ $2.30[1.74,3.04])$, anxiety (OR $=1.21[0.99,1.47])$, and PTSD $(\mathrm{OR}=1.26[1.09,1.46])$ symptoms), and depressive symptoms are consistently one of the strongest risk factors for suicidality $(11,120-123)$.

Within the first component of the stress-coping framework, multiple variable models were run for hypothesized stressors (adverse childhood experiences, traumatic events, discrimination, perceived stress, historical loss, generational out-of-home placement, grief, parental control, and depressive symptoms). For the second component of the stress-coping model, multiple variable models were run for hypothesized protective factors (boarding school attendance, social support, self-esteem, spirituality, resilience, gratitude, enculturation, and constructive coping skills).

Last, full stress-coping models were run with and without depressive symptoms and then with commonly used and culturally relevant control variables (age group, gender, mother's education, current household income, where participant spent their childhood). There were no significant differences in key demographic characteristics when separated into missing and nonmissing samples. 


\subsection{Results}

\subsubsection{Prevalence of Suicidality and Suicide Risk Scores}

Appendix C; Table 1 provides lifetime and past 12-month prevalence estimates for suicidal thoughts and behaviors and suicide risk scores. Results show that $50.8 \%(n=95)$ of the participants reported no suicidal thoughts or behaviors. For lifetime suicidal thoughts and behaviors, $51.7 \%(n=89)$ of the participants reported ideation; 32.4\% $(n=60)$ reported planning an attempt; $26.0 \%(n=46)$ reported telling someone they were thinking of attempting suicide; and, $26.9 \%(n=49)$ reported at least one attempt in their lifetime. Of those who reported a lifetime attempt, $12.1 \%(n=22)$ reported attempting more than one time. Compared to lifetime suicidal thoughts and behaviors, about half as many participants reported thinking about attempting in the past 12 months ( $n=47 ; 26.9 \%)$; however, planning, telling, and actually attempting were much lower. Suicide risk scores ranged from 0-10 with a mean score of 2.12 $(S D=2.51)$. No one received the maximum score of 11 .

\subsubsection{Socio-Demographic Characteristics}

Appendix C; Table 2 presents prevalence estimates, bivariate odds ratios, and $95 \%$ confidence intervals $(\mathrm{Cl})$ for socio-demographic characteristics. Participants in the current analyses included 119 (64.7\%) women and 65 (35.3\%) men. About half ( $n=102 ; 54.6 \%)$ were aged 18-24 and $85(45.5 \%)$ were 25 years of age or older. About 55\% ( $n=98)$ had incomes less than $\$ 10,000 /$ year; $40.1 \%(n=75)$ reported that their mother had a high school degree or less. A majority of the participants $(n=159 ; 85.0 \%)$ reported they spent most of their childhood on or near a reservation. Of the control variables, only higher current household income was significantly associated (inversely) with suicide risk (OR $=0.87$ [0.83, 0.92]).

Fourteen percent $(n=26)$ of the participants reported attending boarding school; $15.1 \%$ ( $n=28)$ and $9.4 \%(n=18)$ of them reported being placed in foster care or adopted, respectively. Twenty-seven percent $(n=50)$ of the participants reported that their mother had attended boarding school and 11.8\% $(n=22)$ reported that she had been placed in foster care or adopted. Compared to their mothers, a smaller percent of the participants reported that their fathers $(18.1 \% ; n=33)$ had gone to boarding school, while a similar percent reported their fathers were placed outside the home $(9.7 \% ; n=18)$.

Unexpectedly, participant boarding school attendance $(\mathrm{OR}=0.67[0.47,0.95])$ was inversely associated with suicide risk. In contrast, both participant foster care (OR $=1.44[1.08$, 1.92]) and adoption $(O R=1.57[1.11,2.23])$ were significantly associated with suicide risk. 
Results varied for their parents with father's boarding school attendance $(\mathrm{OR}=1.34[1.02$, 1.76]) and mother's foster care/adoption (OR = 1.62 [1.19, 2.21]) being significantly associated with participant suicide risk. Neither grandparent's boarding school attendance nor foster care/adoption were significantly associated with participant's suicide risk. However, there was a significant association between participant suicide risk and generational out-of-home placement $(\mathrm{OR}=1.06[1.01,1.13])$.

\subsubsection{Scale Psychometrics and Bivariate Models}

Appendix C; Table 3 provides information on scales and indices (range, mean, standard deviation, Cronbach's $\alpha$ ) used in the analyses, bivariate odds ratios, and $95 \%$ Cls for the scales and indices. The strongest statistically significant positive associations with suicide risk were adverse childhood experiences (OR = 9.32 [5.71, 15.23]), lifetime traumatic events $(O R=11.82$ $[6.53,21.38]$ ), depressive symptoms (OR = $3.36[2.76,4.10])$, perceived stress (OR = 2.19 $[1.83,2.62])$, and anxiety symptoms (OR $=2.01[1.83,2.42])$. Three scales were significantly and inversely associated with suicide risk in the bivariate models: self-esteem $(\mathrm{OR}=0.46[0.37$, $0.56])$, gratitude $(\mathrm{OR}=0.70[0.64,0.78])$, and social support $(\mathrm{OR}=0.90[0.84,0.96])$.

Appendix C; Table 3 also shows results for bivariate odds ratios and $95 \% \mathrm{Cl}$ for individual coping subscales and the two coping factors. Several of the individual subscales were positively and significantly associated with suicide risk (active, humor, religious/spiritual, selfdistraction, denial, venting, substance use, disengagement, self-blame). The two support subscales were significantly and inversely associated with suicide risk. For the two coping factors, non-constructive coping was a statistically significant (positive) risk factor for suicide risk $(\mathrm{OR}=3.22$ [2.61, 3.99]); constructive coping was not.

\subsubsection{Principal Component Factor Loadings for Brief COPE Subscales}

Appendix C; Table 4 provides factor loadings for the Brief COPE subscale principal component analysis, after being constrained to retain two factors suggesting constructive and non-constructive coping. Except for humor, which loaded on both factors (.38; .48), the remaining subscales loaded more strongly on one factor than the other. Since the strongest loading for humor was for the non-constructive coping factor, humor was included in the nonconstructive coping factor. In addition, when the PCA was run with the 28 individual items (as opposed to the 14 subscales), the two humor items loaded more strongly on the nonconstructive coping factor. For brevity, Appendix C; Table 4 displays factor loadings for the 14 subscales only. 


\subsubsection{Multiple Variable Stressor Models}

Appendix C; Table 5 presents results for multiple variable models for the first component of the stress-coping model for suicide risk: the hypothesized stressors. Childhood adversity $(A C E:$ OR $=3.91[2.11,7.22])$ and lifetime traumatic events $(O R=2.23[1.01,4.93])$ showed the strongest associations for suicide risk, even after depressive symptoms were added to the model (Appendix C; Table 5, Model 4). In Models 1 and 2 (Appendix C; Table 5), mother's parental control, discrimination, perceived stress, and thinking about historical losses also were positively and significantly associated with suicide risk. However, perceived stress lost significance after depressive symptoms were added to the model (Appendix C; Table 5, Model 3). There was a significant inverse association between generational out-of-home placement and suicide risk that lost significance when depressive symptoms were added to the model (Model 3).

\subsubsection{Multiple Variable Protective Factor Models}

Appendix C; Table 6 presents results for multiple variable models for the second component of the stress-coping model for suicide risk: the hypothesized protective factors. Except for American Indian identity, all of the factors in Model 1 (Appendix C; Table 6), including participant boarding school attendance, were statistically significantly and inversely associated with suicide risk. However, contrary to the hypothesis, two factors were positively, rather than inversely, associated with suicide risk: spirituality $(O R=1.36[1.20,1.54])$ and resilience $(O R=$ $1.46[1.27,1.69])$. As with the bivariate models, non-constructive coping (OR = 1.85 [1.42, 2.49]) was positively and statistically significantly associated with suicide risk; constructive coping was not significantly associated with suicide risk (Appendix C; Table 6, Model 2).

\subsubsection{Full Stress-Coping Models}

Results for the full stress-coping models are presented in Appendix C; Table 7. Discrimination lost significance when the hypothesized protective factors were added to the model (Appendix C; Table 7, Model 1); no other stressors were affected. However, when depressive symptoms were added to the model (Appendix C; Table 7, Model 2), thinking about historical losses and self-esteem also lost significance. When the coping factors were added to the model, depressive symptoms lost significance (Appendix C; Table 7, Model 4); the traumatic events inventory also lost significance.

Five control variables were added to the full stress-coping model separately: gender, age group, current household income, mother's education level, and childhood home. Although 
gender (Appendix C; Table 8, Model 1) was not a significant factor for suicide risk, the traumatic events inventory, generational out-of-home placement, and resilience lost significance when the full stress-coping model was controlled for gender. As expected, the higher age group was inversely associated with suicide risk in the full stress-coping model (Appendix C; Table 8, Model 2), although, except for resilience, its addition to the model did not affect the independent variables. As in the bivariate models, higher current income was inversely and significantly associated with suicide risk in the full stress-coping model (Appendix C; Table 8, Model 3). In addition, resilience lost significance when the model was controlled for current income. When the model was controlled for mother's education level (Appendix C; Table 8, Model 4), thinking about historical losses regained the significance it had lost when depressive symptoms were added to the stress-coping model (Table 7). Mother's education level also appears to influence generational out-of-home placement and resilience as correlates of suicide risk. Childhood home (Appendix C; Table 8, Model 5) was not a significant factor in the full model and did not alter the significance of the other independent variables.

\subsubsection{Participant Reaction to Participating}

Last, results (not shown) for participant reactions to participating in the survey indicate that although $31.5 \%(n=56)$ reported that completing the survey made them feel upset (somewhat or a great deal), the majority $(88.3 \% ; n=159)$ reported that completing the survey was worth the time (somewhat or a great deal) and $72.5 \%(n=129)$ reported that they felt free to skip questions or to stop at any time (somewhat or a great deal).

\subsection{Discussion}

The prevalence of past 12-month suicide attempts in this tribal college population was remarkably low. This is in contrast to previous studies in American Indian high school students from the same tribal region $(412,459)$; however, it is similar to prevalence estimates found in a population-based sample of tribal members from two Northern Plains communities (121). Study findings are consistent with research that shows that, in contrast to White populations, suicide risk decreases with age in American Indian populations (90). However, while prevalence of current attempts was low in this group, current suicidal thoughts were not. In fact, ideation in the tribal college population was comparable to the American Indian high school population data previously mentioned $(412,459)$. This would suggest the presence of a protective influence in the tribal college population that lessens their risk of progressing from ideation to actually 
attempting suicide. This is noteworthy since more than $50 \%$ of the tribal college students are in the more at-risk age group. Understanding how the tribal college population differs from the general tribal population may be critical for prevention efforts. However, the protective factor in this population may be the fact that they are attending college. For example, attending college may be an indication of self-continuity, in that these students believe in a future and are creating possibilities for a future by pursuing higher education. Thus the tribal college population may not be representative of this age group as a whole; therefore these results may be conservative estimates of suicide risk for this age group. In addition, the tribal colleges foster cultural knowledge and traditional values, which this research indicates may buffer suicide risk in this population (220). These schools also provide an environment which is conducive for advanced education, which is not always valued in tribal communities (personal communication).

Framed within a stress-coping model, the results show high levels of traumatic adversity and other life stressors that were positively associated with suicide risk with less distinct evidence reflecting the influence of proposed protective factors, cultural buffers, and coping strategies. The data support the first hypothesis that higher levels of life stress and poor mental health would be positively associated with suicide risk, although not all stressors retained statistical significance in the full stress-coping models.

Traumatic adversities, especially adverse childhood experiences (ACE), showed the strongest magnitude of association for suicide risk in the full stress-coping models. While the traumatic events inventory lost significance when the model was controlled for gender, controlling for gender did not affect the significance of ACE as a risk factor for suicide risk and only slightly decreased the strength of association of ACE with suicide risk. The level of traumatic stress reported in this population was considerably higher than general population studies $(131,460)$.

Early life traumas can increase the risk of exposure to subsequent traumas and may negatively affect development of constructive coping skills, self-esteem, and cultural identity, which in turn may increase suicide risk $(125,126,129)$. In addition, childhood traumas often cooccur and cumulative ACE have demonstrated a dose-response relation for many suiciderelated risk factors, including an increased risk for depression, substance abuse, and intimate partner violence in American Indians and the general population overall (125, 127, 128, 132, $133,135,142,434,461-466)$. Within First Nation adults, research has shown a link between ACE and increased depressive symptoms, especially in the presence of discrimination, poor coping skills, and negative social support (467). Even though early adversity may influence vulnerability to cope with current stressors, increasing constructive coping and addressing 
healthy relationships are potential areas for intervention as one moves towards adulthood and later life. For example, ACE have been linked to abusive parenting of one's own children (259).

In Native communities, historical events have radically altered the social structure, including assimilation policies, such as mandatory boarding school attendance and urban relocations, as well as the high levels of Native children currently placed outside the home and exorbitantly high rates of unemployment and incarceration. This social disintegration has disrupted family relationships and kinship structure, inhibiting the generational transmission of language, traditional parenting skills, and cultural practices, thereby weakening the collective conscience deemed important by Durkheim for strong social integration that is protective against suicide risk $(28,29)$. The kinship structure and collective social structure provided protection for women as well as children; family violence was rare in traditional Native society (468). Changes in parenting style and current high levels of family violence are a reflection of the disrupted attachments (31).

Previous studies have shown that thinking about historical losses was positively associated with alcohol abuse, anger, and symptoms of internalization in adults and depressive symptoms in adolescents $(200,246)$. It is important to note that when combined with depressive symptoms, these thoughts were not significantly associated with suicide risk in the full stresscoping model in the current study. However, grief had also been removed during model reduction. More research is needed to quantify the connection between thinking about historical losses and grief, especially unresolved grief, which is in line with the historical trauma literature (469).

Current high levels of discrimination reported in this study also are problematic for suicide risk. However, the effect was mitigated when protective factors were added to the model. More research is needed to determine which factors provide the most protection and which types of discrimination are most negatively associated with poor mental health and suicide risk to effectively focus prevention efforts.

Data from the current study partially support the second hypothesis that higher levels of enculturation, resilience, constructive coping skills, social support, self-esteem, and gratitude mitigate suicide risk. This is evident in that not all stressors retained statistical significance in the full stress-coping models, which may be indicative of a buffering effect from some of the proposed protective factors. However, contrary to the second hypothesis, resilience and spirituality were positively, rather than inversely, associated with suicide risk.

Ironically, the high level of childhood and later life adversity in this tribal college population may account for the positive association of resilience with suicide risk. By definition, 
resilience increases as a result of successfully adapting to adversity; however, research has shown a quadratic, rather than linear relation, between cumulative adversity and mental health and well-being outcomes (470). It may be that the level of adversity in this population has passed the critical point of successful adaption, where the ability to constructively cope with adversity lessens. More research is needed to determine whether the effect of resilience and ability to cope adaptively vary with level of cumulative adversity, gender, and age group.

Although the protective influence of participant boarding school attendance (as opposed to parent or grandparent attendance) was unexpected, this makes sense in light of education policy changes begun in the 1970s. The institution of the Indian Self-Determination and Education Acts of the 1970s and the Indian Child Protection Act of 1990 allowed tribes to assume more responsibility for their children's education, including stringent background checks for teachers and staff to help protect children. Results from this study indicate that these schools now appear to provide a protective environment against discrimination and abusive home environments $(77,208,212,219)$. This is consistent with Durkheim's social integration and evidenced by research in British Columbia bands that shows that higher levels of cultural continuity, such as markers of self-determination and tribal autonomy, are protective against suicide risk in First Nations youth $(28,29,212)$.

If youth suicide is related to disrupted development, it stands to reason that being involved in and identifying with one's culture may be a key protective factor, since culture provides a foundation that gives meaning to life and a way to see oneself moving into the future (316). However, in the current study, American Indian identity was not protective against suicide risk. This may be a result of sample size or older average age in tribal college participants. It also may be more meaningful to focus on specific cultural values, such as gratitude and generosity, as opposed to items that measure level of involvement in American Indian way of life, because gratitude was consistently inversely associated with suicide risk in this study. This may also explain the positive association of spirituality with suicide risk. Perceptions of commitment to or finding comfort or guidance in religion, as with cultural involvement, may be extrinsic measures of an orientation. Whereas gratitude may be an intrinsic, internalized expression of spirituality and cultural values, and therefore may be a more meaningful measure (471). Although more research is needed in this area, gratitude toward God has shown a buffering effect on stress and poor health in a nationwide sample of older adults, especially women (472).

The main strength of the current study is the information gained by testing a stresscoping model of culturally relevant stressors and protective factors in a unique tribal population. 
Both elements add to the literature and provide avenues for more in-depth research, as well as implications for intervention development. This is highly innovative, because to our knowledge, a stress-coping model for suicide risk has not been tested in an American Indian population.

The study is not without limitations. The small sample size limited the number of variables that could be included in the models. The results for independent variables in this sample that were statistically significant can be trusted; however, the non-significant variables may be affected by sample size and should be interpreted with caution. The sample size also did not allow for a more in-depth examination of anxiety and PTSD symptoms. In addition, we were unable to stratify by gender. Gender stratification is important for several reasons. Women and men are often exposed to different types of adversity and also appraise and cope with adversity in different ways $(38,39,138)$. The study also was limited to one tribal region. Including tribal colleges nation-wide would provide a larger sample for regional, as well as gender, comparisons. Other limitations include recall bias and causal direction limits found with cross-sectional data. Last, mixed-mode data collection may introduce measurement error (473). However, there were no significant differences in key demographic characteristics between the on-line and paper surveys in the analytic sample.

In conclusion, testing a stress-coping model has provided important information on associations between general and culturally relevant stressors and protective factors for suicide risk in this unique tribal college setting. Results highlight the importance of examining both stressors and protective factors. While inclusion of protective factors did not eliminate the influence of childhood adversity, the results have implications for socioeconomic, structural, family, and culture-based intervention development.

The study results also indicate promising areas for future research related to protective factors, including education policy analysis and examination of cultural values, such as gratitude, as well as differentiating between which factors mitigated the effect of discrimination on suicide risk. Future research informed and driven by community identified needs is important to address unanswered questions such as why certain coping strategies were preferred, whether there are gender differences in preference, and if other, perhaps more culturally relevant strategies, should be included in the measure. Because humor loaded as both a constructive and non-constructive coping strategy, it is important for future research to examine the effect of different types of humor on suicide risk in American Indians (474). Communitybased approaches also are needed to better understand resilience in American Indian populations. More research is needed in tribal college populations to understand the apparent protective mechanisms at play in their ability to overcome adversity to help reduce morbidity and 
mortality reservation-wide. Doing so will promote strong, healthy future generations while informing prevention efforts for this high-risk population. 


\section{Chapter 5. Summary of Research}

\subsection{Summary}

Suicidality is a complex phenomenon and a distressing indicator of the mental suffering, disrupted attachments, and social disintegration found in many Native communities (31, 48, 232). Recent research indicates significant downward trends in age-adjusted suicide rates in 10 24 year old persons nationwide, including in American Indian populations where the rates are still the highest of all US racial/ethnic groups (475). Results from this dissertation research provide positive areas for culturally relevant suicide prevention and intervention to continue this downward trend.

The goal of this dissertation research was to examine culturally unique psychosocial risk and protective factors and coping strategies associated with suicidality in American Indian youth and young adults. To accomplish this goal, three interdependent cross-sectional studies were undertaken. The first two studies used secondary data from the Youth Risk Behavior Survey (YRBS) and the third study used primary data from an original survey designed in collaboration with tribal members collected in a unique tribal college setting. The three studies are described in detail in Chapters 2-4. The focus of Chapter 2 (Specific Aim 1; Study 1) was to identify gender-specific individual-level YRBS risk factors associated with suicidal ideation/attempts for American Indian and White Montana high school students using pooled random sample YRBS data. The focus of Chapter 3 (Specific Aim 2; Study 2) was to identify gender-specific individuallevel YRBS risk factors associated with both suicidal ideation and suicide attempts in American Indian students attending Montana reservation and urban high schools using pooled volunteer sample YRBS data. The focus of Chapter 4 (Specific Aim 3; Study 3) was to test a stress-coping model of the relation between life stress, coping skills, enculturation, cultural values, resilience, psychological well-being, social support, and perceived parenting skills on suicide risk in American Indian tribal college students using primary survey data.

Results from the examination of gender-specific risk factors for American Indian and White youth (Specific Aim 1; Chapter 2) confirmed the significantly higher prevalence of suicidal ideation and attempts and depressive symptoms in American Indian girls and boys compared to White boys and girls found in previous research $(78,80,87)$. The American Indian youth consistently reported significantly higher levels of adversity (dating violence, sexual assault, feeling unsafe at school) and non-constructive coping (substance use, unhealthy weight control) than the White youth (Appendix A; Table 1). Sadness/hopelessness, the only depressive 
symptom available in the YRBS, was the only risk factor that was significantly associated with suicidal ideation/attempts for the American Indian and White girls and boys. In addition to sadness/hopelessness, only one other risk factor was significantly associated with suicidal ideation/attempts for the American Indian girls (unhealthy weight control) and boys (weapon carrying). In contrast, for the White girls and boys, many of the YRBS risk factors showed a significant association with suicidal ideation/attempts (Appendix A; Table 2).

Based on reservation/urban location, data from Specific Aim 2 (Study 2; Chapter 3) revealed no significant differences in prevalence of suicidal ideation and suicide attempts in the reservation/urban locations for either gender (Appendix B; Table 1). However, there were differences in significant risk factors for ideation and attempts (Appendix B; Tables 2-5). The urban girls in this study reported significantly more sadness/hopelessness, partner victimization, injection drug use, and weapon carrying than the reservation girls; the reservation girls reported significantly more alcohol/tobacco/marijuana use than the urban girls. For the boys, significant differences in the prevalence of risk factors were primarily found in type of substance use reported. Results from Study 2 (Chapter 3) also showed gender differences in significant risk factors for suicidal ideation and attempts across the reservation/urban locations with fewer differences noted for girls than boys (Appendix B; Tables 2-5). Sadness/hopelessness (for all the youth) and partner victimization (for all except the urban boys) were significant risk factors for ideation along with some type of coping strategy (for the reservation, but not the urban, youth). The significant risk factors for suicide attempts in the girls were similar, including sadness/hopelessness, victimization (partner violence, lack of school safety), and coping strategies (unhealthy weight control, early risk-taking, weapon carrying). Sadness/hopelessness and lack of school safety were the only common significant risk factors for suicide attempts for the reservation and urban boys and the only two significant risk factors for the reservation boys. For the urban boys, partner victimization, unhealthy weight control, and early risk-taking also were significantly associated with suicide attempts.

Specific Aim 3 (Study 3; Chapter 4) results were based on primary data collected in Montana-Wyoming tribal colleges. An important finding is the low level of past year suicide risk reported in this American Indian (self-identified) tribal college population, in particular, low levels of past year reported suicide attempts (Appendix C; Table 1). Fourteen percent of the participants in this study reported attending boarding school, which showed an unexpected inverse association with suicide risk (Appendix C; Table 2). We looked at two categories of generational out-of-home placement as potential risk factors for suicide risk: boarding school placement and foster care/adoption. In the bivariate models (Appendix C; Table 2), for boarding 
school placement, father's boarding school attendance was the only significant risk factor for suicide risk; foster care/adoption was a significant risk factor for suicide risk for the participant and the participant's mother. Grandparent out-of-home placement was not a significant risk factor in the bivariate models. When the two categories were combined across the generations, as expected, there was a significant positive association with suicide risk in the bivariate models; unexpectedly, this association inversed in the stressor models, although the association did not remain significant in the full stress-coping models (Appendix C; Tables 5, 7, and 8).

Within the first component of the stress-coping model, all of the risk factors (stressors) were positively and statistically significantly associated with suicide risk (Appendix C; Table 3). The strongest associations were found for lifetime traumatic events and adverse childhood experiences (ACE), as well as mental health symptoms (depression, anxiety, perceived stress, posttraumatic stress disorder). ACE and lifetime traumatic events remained the most significant risk factors for suicide risk in this tribal college population in the multiple variable stressor (risk factor) models (Appendix C; Table 5). Within the second component of the stress-coping model, the protective factor model (Appendix C; Table 6), participant boarding school attendance, social support, self-esteem, and gratitude were inversely associated with suicide risk; unexpectedly, higher levels of spirituality and resilience were positively associated with suicide risk. As expected, non-constructive coping was positively associated with suicide risk, while constructive coping was not significantly associated with suicide risk.

The full stress-coping models (Appendix C; Table 7) show the potential benefit of protective factors, because depressive symptoms, discrimination, and generational out-of-home placement lost significance after the protective factors were added to the model (Appendix C; Table 7, Model 1 and 2). Thinking about historical losses lost significance when depressive symptoms were added to the model; self-esteem did as well (Appendix C; Table 7, Model 2). Again, loss of significance may be indicative of the limited sample size for the number of independent variables. Participant boarding school attendance, social support, and gratitude were consistently inversely associated with suicide risk across the stress-coping models (Appendix C; Table 7), even after controlling for gender, age, current income, mother's education, and childhood home (Appendix C; Table 8).

\subsection{Strengths and Limitations}

Two of these studies, using YRBS data, were limited to assessing risk factors. Even though YRBS data are not always culturally relevant for assessing suicide risk in Native youth, 
the first two studies confirmed the higher prevalence of suicidality in girls compared to boys and the higher prevalence of suicidality in American Indian youth compared to White youth found in previous research.

Study 2 results showed no significant differences in ideation or attempts across the reservation/urban locations for either gender; however, there were differences in significant risk factors across the reservation/urban locations. Reservation life may confer some protective benefit, especially in terms of the prevalence of sexual assault, i.e., childhood adversity; however, the prevalence of sexual assault reported by reservation youth is still significantly higher than estimates reported by White youth in the same geographic area. Some of the violent victimization may be related to discrimination.

Although limited by the risk factors that the YRBS data allowed us to analyze, the results from the two YRBS studies, in conjunction with the focus group results from the Planting Seeds of Hope youth suicide prevention evaluation, provided a foundation for developing the comprehensive and culturally relevant stress-coping survey conducted with the MontanaWyoming tribal college students.

The tribal college study (Specific Aim 3; Study 3, Chapter 4) was limited by the small sample size. However, use of the culturally relevant model, developed in consultation with tribal members confirmed risk factors not found in YRBS data, such as discrimination, found in other settings and racial/ethnic groups $(113,164-166)$. These results also add to limited research regarding the value of protective factors and provide important information that is pivotal in designing culturally relevant interventions for suicide prevention within Native populations.

The main strength of the tribal college study (Chapter 4) is testing a stress-coping model of culturally relevant stressors and protective factors in a unique tribal population. Both elements are innovative and address gaps in the literature, thereby providing avenues for more in-depth research, as well as implications for intervention development. This is highly innovative, because to our knowledge, a stress-coping model for suicide risk has not been tested in an American Indian population.

However, the tribal college study is not without limitations. The small sample size limited the number of variables that could be included in the models and did not allow for a more indepth examination of anxiety and PTSD symptoms. We also were unable to stratify by gender, which may be important for intervention development. A larger, more regionally diverse sample would allow us to examine regional and gender differences in risk and protective factors. The study also was limited by recall bias and causal direction limits found with cross-sectional data. 
In conclusion, testing a stress-coping model has provided important information on associations between general and culturally relevant stressors and protective factors for suicide risk in this unique tribal college setting. Results highlight the importance of examining both stressors and protective factors which have implications for socioeconomic, structural, family, and culture-based intervention development.

\subsection{Future Research}

The dissertation results indicate promising areas for future research. For example, through this seven-year process we found that community-based approaches are needed to better understand resilience and coping strategies in American Indian populations. A more indepth look at types of discrimination and who perpetrates the discriminatory acts, for example whether Native or non-Native, would help inform interventions. It also is important to understand if there is a link between discrimination and violent victimization in Native youth, which directly relates to suicidality among all youth, but in particular Native youth because the prevalence of violent victimization is significantly higher for Native compared to other youth $(78,80,412)$. In line with the historical trauma literature, more research is needed to quantify the connection between thinking about historical losses and grief, especially unresolved grief. More protective factor research is needed, including how cultural values inform resilience and their effect on positive mental health. More research is needed to determine why generational out-of-home placement was inversely associated with suicide risk in all but the final stressor models (Appendix C; Table 5, Model 4). Policy analysis is important to understand its effects on American Indian health and to inform future policies aimed at reducing disparate rates of suicide and related risk factors among Native youth and young adults. Evaluating prevention and intervention programs for the unique cultural context also is needed. Combining these efforts will build an evidence base of what works in Indian Country to reduce suicidality among Native youth and build on healthy, resilient, future generations.

More research is needed in tribal college populations to understand the apparent protective mechanisms at play in their ability to overcome adversity to help reduce suiciderelated morbidity and mortality reservation-wide. This type of research would help us better understand how to prevent middle and high school age American Indians from progressing from suicidal ideation to actually attempting. Understanding the factors that most strongly influence positive mental health in the tribal college population is an important step in future research that builds on generalized and culturally unique protective factors $(21,476-478)$. Research with a 
larger, more regionally diverse tribal college sample would allow us to examine regional and gender differences in risk and protective factors, including a more in-depth examination of anxiety and PTSD symptoms.

\subsection{Conclusions and Significance}

This dissertation research contributes to the literature by confirming previous research comparing American Indian and White youth and by showing differences in risk factors for both suicidal ideation and suicide attempt outcomes based on reservation versus urban location. It also contributes to the literature by testing a stress-coping model for suicide risk, which to our knowledge, had not previously been done in an American Indian population. These results have important implications for effective intervention development: by integrating protective factors, Native populations may effectively use cultural resources when designing programs and enacting policies protective against suicidality among American Indian youth and young adults.

The goal of this research was to address gaps in the literature. This goal was reached by expanding knowledge of risk factors for suicidal ideation and attempts in American Indian youth in an under-researched tribal area. This research also broadened the field by examining the association between suicide risk and culturally unique risk and protective factors in American Indian tribal college students. This fills an important gap because limited suicide research has been conducted with American Indian college students and to our knowledge this is the first suicide-related study to be conducted with tribal college students.

Results from Study 1 and Study 2 show that it is important for interventions to be tailored to meet the different needs of boys and girls, as well as the different needs of American Indian youth who reside predominantly in reservation, as opposed to urban, areas.

Results from the three studies combined show that the prevalence of stressors, such as childhood adversity and discrimination, are quite high in this population. Results from Study 2 show that reservation life may confer some protective benefit; however, growing up on the reservation did not affect the significance of any of the independent variables in Study 3 . This may be due to a lack of variance, because $85 \%$ of the participants in Study 3 reported that they grew up on a reservation. The results from Study 3 demonstrate the importance of including culturally relevant risk and protective factors in the YRBS. These studies indicate the importance of teaching life skills, constructive coping, and instilling cultural values in American Indian youth and young adults. These teachings are warranted because the high levels of 
trauma in this population may tax the ability of youth and young adults to successfully cope with life stressors.

These results have implications for development of culturally relevant interventions that have the potential to lessen the amount of childhood adversity present in the population and those that help increase constructive coping skills to help mitigate the effects of experiencing this adversity, and in turn, reducing suicide risk. In particular, the results provide empirical evidence to support the necessity of widely used Native-developed interventions, such as Healthy Relationships and American Indian Life Skills. Developed for Native adults, the dissertation data support the need for adapting the Healthy Relationships program for Native youth and also adapting the youth-focused American Indian Life Skills program for Native adults. These culturally relevant interventions were designed to help heal the disrupted attachments and resultant unhealthy coping mechanisms found in many tribal communities by addressing life skills, traditional parenting skills, and healthy relationships that build on cultural values, such as gratitude, generosity, and humility as well as relationships that are based on the premise that women and children are sacred and not to be abused. Increasing healthy coping skills also could help American Indian youth and young adults constructively cope with social stressors, such as experiencing discrimination and feeling a lack of safety at school. School screenings for mental health, dating violence, school safety, and substance use are warranted, as well as referral to culturally meaningful and gender-specific counseling, as well as programs such as American Indian Life Skills. Because there are few evidenced-based culturally relevant interventions, the results from this dissertation can be used to help secure funding to build an evidence-base and advance community-level capacity to most effectively implement these programs.

In conclusion, American Indian youth and young adults are at great risk for suicide and suicide-related risk factors. Knowing they are at increased risk is of utmost importance for current and future suicide prevention programs aimed at protecting American Indian populations. This research provides valuable information to enhance intervention efforts to meet the unique needs of this population. American Indian youth and young adults must be given the opportunity to reach their full potential - for the benefit of themselves, their families, and future healthy generations. 


\section{REFERENCES}

1. Anisman $\mathrm{H}$, Merali Z. Understanding stress: Characteristics and caveats. Alcohol Res Health. 1999;23(4):241-9.

2. Diagnostic and Statistical Manual of Mental Disorders, Fifth Edition (DSM-5). American Psychiatric Association, editor. Arlington, VA:2013.

3. Durkheim E. Suicide: A Study in Sociology. 1979 ed. New York: The Free Press; 1897.

4. Baldessarini RJ, Hennen J. Genetics of suicide: An overview. Harv Rev Psychiatry. 2004;12(1):1-13 doi: 10.1080/10673220490425915.

5. Brezo J, Klempan T, Turecki G. The genetics of suicide: A critical review of molecular studies. Psychiatr Clin North Am. 2008;31(2):179-203. doi: 10.1016/j.psc.2008.01.008.

6. McGowan PO, Sasaki A, Huang TC, Unterberger A, Suderman M, Ernst C, Meaney MJ, Turecki G, Szyf M. Promoter-wide hypermethylation of the ribosomal RNA gene promoter in the suicide brain. PLoS One. 2008;3(5):e2085. doi: 10.1371/journal.pone.0002085.

7. Brezo J, Bureau A, Merette C, Jomphe V, Barker ED, Vitaro F, Hebert M, Carbonneau R, Tremblay RE, Turecki G. Differences and similarities in the serotonergic diathesis for suicide attempts and mood disorders: A 22-year longitudinal gene-environment study. Mol Psychiatry. 2010;15(8):831-43. doi: 10.1038/mp.2009.19.

8. Guintivano J, Brown T, Newcomer A, Jones M, Cox O, Maher BS, Eaton WW, Payne JL, Wilcox HC, Kaminsky ZA. Identification and replication of a combined epigenetic and genetic biomarker predicting suicide and suicidal behaviors. Am J Psychiatry. 2014;171(12):1287-96. doi: 10.1176/appi.ajp.2014.14010008.

9. Costanza A, D'Orta I, Perroud N, Burkhardt S, Malafosse A, Mangin P, La Harpe R. Neurobiology of suicide: Do biomarkers exist? Int J Legal Med. 2014;128(1):73-82. doi: 10.1007/s00414-013-0835-6.

10. Mullins N, Perroud N, Uher R, Butler AW, Cohen-Woods S, Rivera M, Malki K, Euesden J, Power RA, Tansey KE, Jones L, Jones I, Craddock N, Owen MJ, Korszun A, Gill M, Mors O, Preisig M, Maier W, Rietschel M, Rice JP, Müller-Myhsok B, Binder EB, Lucae S, Ising M, Craig IW, Farmer AE, McGuffin P, Breen G, Lewis CM. Genetic relationships between suicide attempts, suicidal ideation and major psychiatric disorders: A genome-wide association and polygenic scoring study. Am J Med Genet. 2014;Part B9999:1-10.

11. Foley DL, Goldston DB, Costello EJ, Angold A. Proximal psychiatric risk factors for suicidality in youth: The Great Smoky Mountains Study. Arch Gen Psychiatry. 2006;63(9):101724. doi: 10.1001/archpsyc.63.9.1017.

12. Nock MK, Hwang I, Sampson N, Kessler RC, Angermeyer M, Beautrais A, Borges G, Bromet E, Bruffaerts R, de Girolamo G, de Graaf R, Florescu S, Gureje O, Haro JM, Hu C, Huang Y, Karam EG, Kawakami N, Kovess V, Levinson D, Posada-Villa J, Sagar R, Tomov T, Viana MC, Williams DR. Cross-national analysis of the associations among mental disorders 
and suicidal behavior: Findings from the WHO World Mental Health Surveys. PLoS Med. 2009;6(8):e1000123. doi: 10.1371/journal.pmed.1000123.

13. Ball L, Chandler M. Identity formation in suicidal and nonsuicidal youth: The role of selfcontinuity. Development and Psychopathology. 1989;1(3):257-75.

14. De Leo D, Padoani W, Scocco P, Lie D, Bille-Brahe U, Arensman E, Hjelmeland H, Crepet P, Haring C, Hawton K, Lonnqvist J, Michel K, Pommereau X, Querejeta I, Phillipe J, Salander-Renberg E, Schmidtke A, Fricke S, Weinacker B, Tamesvary B, Wasserman D, Faria S. Attempted and completed suicide in older subjects: Results from the WHO/EURO Multicentre Study of Suicidal Behaviour. Int J Geriatr Psychiatry. 2001;16(3):300-10. doi: 10.1002/gps.337.

15. Chandler M. Self-continuity in suicidal and nonsuicidal adolscents. New Directions for Child Development. 1994;64(Summer):55-70.

16. Chandler MJ. The time of our lives: Self-continuity in Native and non-Native youth. Adv Child Dev Behav. 2001;28:175-221.

17. Rockett IR, Regier MD, Kapusta ND, Coben JH, Miller TR, Hanzlick RL, Todd KH, Sattin RW, Kennedy LW, Kleinig J, Smith GS. Leading causes of unintentional and intentional injury mortality: United States, 2000-2009. Am J Public Health. 2012;102(11):e84-e92. doi: doi:10.2105/AJPH.2012.300960.

18. Kessler RC, Berglund P, Borges G, Nock M, Wang PS. Trends in suicide ideation, plans, gestures, and attempts in the United States, 1990-1992 to 2001-2003. JAMA. 2005;293(20):2487-95. doi: 10.1001/jama.293.20.2487.

19. McKeown RE, Cuffe SP, Schulz RM. US suicide rates by age group, 1970-2002: An examination of recent trends. Am J Public Health. 2006;96(10):1744-51.

20. Alcantara C, Gone JP. Reviewing suicide in Native American communities: Situating risk and protective factors within a transactional-ecological framework. Death Stud. 2007;31(5):45777.

21. Allen J, Mohatt GV, Fok CCT, Henry D, Burkett R. A protective factors model for alcohol abuse and suicide prevention among Alaska Native youth. Am J Community Psychol. 2014;54(1-2):125-39. doi: 10.1007/s10464-014-9661-3.

22. De Leo D, Milner A. The WHO/START study: Promoting suicide prevention for a diverse range of cultural contexts. Suicide Life Threat Behav. 2010;40:99-106. doi:

10.1521/suli.2010.40.2.99.

23. Rubinstein DH. A stress-diathesis theory of suicide. Suicide Life Threat Behav. 1986;16(2):182-97.

24. Dinges NG, Joos SK. Stress, coping, and health: Models of interaction for Indian and Native populations. Am Indian Alsk Native Ment Health Res. 1988;1:8-55.

25. Walters KL, Simoni JM. Reconceptualizing Native women's health: An "Indigenist" stress-coping model. Am J Public Health. 2002;92(4):520-4. 
26. Manzo K, Stewart J, Gachupin F. A qualitative analysis of factors underlying suicidality in Montana American Indian youth and young adults. Manuscript in Preparation.

27. Ide N, Wyder M, Kolves K, De Leo D. Separation as an important risk factor for suicide: A systematic review. Journal of Family Issues. 2010;31(12):1689-716. doi:

10.1177/0192513X10365317.

28. Chandler MJ, Lalonde C. Cultural continuity as a hedge against suicide in Canada's First Nations. Transcult Psychiatry. 1998;35(2):191-219. doi: 10.1177/136346159803500202.

29. Chandler MJ, Lalonde CE. Cultural continuity as a moderator of suicide risk among Canada's First Nations. In: Kirmayer L, Valaskakis G, editors. Healing Traditions: The Mental Health of Aboriginal Peoples in Canada: University of British Columbia Press; 2008. p. 221-48.

30. Duran E, Duran B. Native American Postcolonial Psychology: State University of New York Press; 1995.

31. Haskell L, Randall M. Disrupted attachments: A social context complex trauma framework and the lives of Aboriginal peoples of Canada. Journal of Aboriginal Health. 2009;5(3):48-99.

32. Yellow Horse Brave Heart M, DeBruyn LM. The American Indian holocaust: Healing historical unresolved grief. Am Indian Alsk Native Ment Health Res. 1998;8(2):56-78.

33. Walters KL, Mohammed SA, Evans-Campbell T, Beltran RE, Chae DH, Duran B. Bodies don't just tell stories, they tell histories: Embodiment of historical trauma among American Indians and Alaska Natives. Du Bois Review. 2011;8(1):179-90.

34. Chagnon F. Coping mechanisms, stressful events and suicidal behavior among youth admitted to juvenile justice and child welfare services. Suicide Life Threat Behav. 2007;37(4):439-52. doi: 10.1521/suli.2007.37.4.439.

35. Grover KE, Green KL, Pettit JW, Monteith LL, Garza MJ, Venta A. Problem solving moderates the effects of life event stress and chronic stress on suicidal behaviors in adolescence. J Clin Psychol. 2009;65(12):1281-90. doi: 10.1002/jclp.20632.

36. Evans-Campbell T. Historical trauma in American Indian/Native Alaska communities: A multilevel framework for exploring impacts on individiuals, families, and communities. J Interpers Violence. 2008;23(3):316-38.

37. Aldwin CM. Developmental studies of coping. Stress, Coping, and Development: An Intergrative Perspective. New York: The Guilford Press; 2007.

38. Matud MP. Gender differences in stress and coping styles. Personality and Individual Differences. 2004;37(7). doi: http://dx.doi.org/10.1016/j.paid.2004.01.010.

39. Stein JA, Nyamathi A. Gender differences in relationships among stress, coping, and health risk behaviors in impoverished, minority populations. Personality and Individual Differences. 1999;26(1):141-57. doi: 10.1016/S0191-8869(98)00104-4. 
40. Aldwin CM. Sociocultural aspects of coping. Stress, Coping, and Development: An Intergrative Perspective. New York, NY: Guilford Press; 2007.

41. Grunebaum MF, Ramsay SR, Galfalvy HC, Ellis SP, Burke AK, Sher L, Printz DJ, Kahn DA, Mann JJ, Oquendo MA. Correlates of suicide attempt history in bipiolar disorder: A stressdiathesis perspective. Bipolar Disorders. 2006;8:551-7.

42. Priester MJ, Clum GA. The problem-solving diathesis in depression, hopelessness, and suicide ideation: A longitudinal analysis. Journal of Psychopathology and Behavioral Assessment. 1993;15(3):239-54.

43. Rich AR, Bonner RL. Concurrent validity of a stress-vulnerability model of suicidal ideation and behavior: A follow-up study. Suicide Life Threat Behav. 1987;17(4):265-70.

44. Kading M, Hautala DS, Palombi LC, Aronson BD, Smith RC, Walls ML. Flourishing: American Indian positive mental health. Society and Mental Health. Epub February 2, 2015. doi: $10.1177 / 2156869315570480$.

45. De Leo D. Struggling against suicide: The need for an integrative approach. Crisis. 2002;23(1):23-31.

46. Lester D. Suicide and culture. World Cultural Psychiatry Research Review. 2008;Apr:5168.

47. Nock MK, Borges G, Bromet EJ, Alonso J, Angermeyer M, Beautrais A, Bruffaerts R, Chiu WT, de Girolamo G, Gluzman S, de Graaf R, Gureje O, Haro JM, Huang Y, Karam E, Kessler RC, Lepine JP, Levinson D, Medina-Mora ME, Ono Y, Posada-Villa J, Williams D. Cross-national prevalence and risk factors for suicidal ideation, plans and attempts. $\mathrm{Br} \mathrm{J}$ Psychiatry. 2008;192(2):98-105. doi: 10.1192/bjp.bp.107.040113.

48. Kirmayer LJ, Brass GM, Holton TL, Paul K, Simpson C, Tait CL. Suicide Among Aboriginal Peoples in Canada. Ottawa, ON: Aboriginal Healing Foundation, 2007.

49. For the Cedar Project Partnership, Moniruzzaman A, Pearce ME, Patel SH, Chavoshi N, Teegee M, Adam W, Christian WM, Henderson E, Craib KJP, Schecter MT, Spittal PM. The Cedar Project: Correlates of attempted suicide among young Aboriginal people who use injection and non-injection drugs in two Canadian cities. Int J Circumpolar Health. 2009;68(3):261-73.

50. LaFromboise TD, Lewis HA. The Zuni Life Skills Development Program: A school/community-based suicide prevention intervention. Suicide Life Threat Behav. 2008;38(3):343-53.

51. Harrop AR, Brant RF, Ghali WA, Macarthur C. Injury mortality rates in Native and nonNative children: A population-based study. Public Health Rep. 2007;122(3):339-46.

52. Hunter E, Harvey D. Indigenous suicide in Australia, New Zealand, Canada and the United States. Emergency Medicine. 2002;14:14-23. 
53. Wexler L, Silveira ML, Bertone-Johnson E. Factors associated with Alaska Native fatal and nonfatal suicidal behaviors 2001-2009: Trends and implications for prevention. Arch Suicide Res. 2012;16(4):273-86. doi: 10.1080/13811118.2013.722051.

54. Lehti V, Niemelä S, Hoven C, Mandell D, Sourander A. Mental health, substance use and suicidal behaviour among young indigenous people in the Arctic: A systematic review. Soc Sci Med. 2009;69(8):1194-203. doi: 10.1016/j.socscimed.2009.07.045.

55. Coloma C, Hoffman JS, Crosby AE. Suicide among Guarani Kaiowa and Nandeva youth in Mato Grosso do Sul, Brazil. Arch Suicide Res. 2006;10:191-207.

56. Bjerregaard P, Lynge I. Suicide: A challenge in modern Greenland. Arch Suicide Res. 2006;10:209-20.

57. Ran MS. Suicide in Micronesia: A systematic review. Primary Psychiatry. 2007;14(11):80-7.

58. Lester D. Suicide among Indigenous peoples: A cross-cultural perspective. Arch Suicide Res. 2006;10:117-24.

59. Sumarokov YA, Brenn T, Kudryavtsev A, Nilssen O. Suicides in the indigenous and nonindigenous populations in the Nenets Autonomous Okrug, Northwestern Russia, and associated socio-demographic characteristics. Int J Circumpolar Health. 2014;73(25308).

60. De Leo D. Suicide and Euthanasia in Older Adults: A Transcultural Journey. Seattle; Toronto: Hogrefe \& Huber; 2001.

61. Nock MK, Borges G, Bromet EJ, Cha CB, Kessler RC, Lee S. Suicide and suicidal behavior. Epidemiol Rev. 2008;30:133-54. doi: 10.1093/epirev/mxn002.

62. Mullany B, Barlow A, Goklish N, Larzelere-Hinton F, Cwik M, Craig M. Toward understanding suicide among youths: Results from the White Mountain Apache tribally mandated suicide surveillance system, 2001-2006. Am J Public Health. 2009;99(10):1840-8. doi: 10.2105/AJPH.2008.154880.

63. Mulye TP, Park MJ, Nelson CD, Adams SH, Irwin CE, Brindis CD. Trends in adolescent and young adult health in the United States. J Adolescent Health. 2009;45:8-24.

64. Dorgan BL. The tragedy of Native American youth suicide. Psychological Services. 2010;7(3):213-8.

65. Drum DJ, Brownson C, Denmark AB, Smith SE. New data on the nature of suicidal crises in college students: Shifting the paradigm. Professional Psychology: Research and Practice. 2009;40(3):213-22.

66. Schwartz AJ. College student suicide in the United States: 1990-1991 through 20032004. Journal of American College Health. 2006;54(6):341-52.

67. Wilcox HC, Arria AM, Caldeira KM, Vincent KB, Pinchevsky GM, O'Grady KE. Prevalence and predictors of persistent suicide ideation, plans, and attempts during college. Journal of Affective Disorders. 2010;127:287-94. 
68. Rockett IR, Hobbs G, De Leo D, Stack S, Frost JL, Ducatman AM, Kapusta ND, Walker RL. Suicide and unintentional poisoning mortality trends in the United States, 1987-2006: Two unrelated phenomena? BMC Public Health. 2010;10. doi: 10.1186/1471-2458-10-705.

69. Adjusting for Miscoding of Indian Race on State Death Certificates. Indian Health Service. Department of Health and Human Services: 1996.

70. Harwell TS, Hansen D, Moore KR, Jeanotte D, Gohdes D, Helgerson SD. Accuracy of race coding on American Indian death certificates, Montana 1996-1998. Public Health Rep. $2002 ; 117(1): 44-9$.

71. CDC. Surveillance for violent deaths: National Violent Death Reporting System, 16 States, 2009. MMWR. 2012;61(ss06).

72. Rockett IRH, Samora JB, Coben JH. The black-white suicide paradox: Possible effects of misclassification. Soc Sci Med. 2006;63:2165-75.

73. Rockett IR, Wang S, Stack S, De Leo D, Frost JL, Ducatman AM, Walker RL, Kapusta ND. Race/ethnicity and potential suicide misclassification: Window on a minority suicide paradox? BMC Psychiatry. 2010;10(35). doi: 10.1186/1471-244X-10-35.

74. Huguet N, Kaplan MS, McFarland BH. Rates and correlates of undetermined deaths among African Americans: Results from the National Violent Death Reporting System. Suicide Life Threat Behav. 2012;42(42):185-96.

75. Keane EM, Dick RW, Bechtold DW, Manson SM. Predictive and concurrent validity of the Suicidal Ideation Questionnaire among American Indian adolescents. J Abnorm Child Psychol. 1996;24(6):735-47.

76. Yuen NYC, Nahulu LB, Hishinuma ES, Miyamoto RH. Cultural identification and attempted suicide in Native Hawaiian adolescents. Journal of the American Academy of Child \& Adolescent Psychiatry. 2000;39(3):360-7.

77. Dinges NG, Duong-Tran Q. Suicide ideation and suicide attempt among American Indian and Alaska Native boarding school adolescents. Am Indian Alsk Native Ment Health Res. 1994;4:167-88.

78. Pavkov TW, Travis L, Fox KA, King CB, Cross TL. Tribal youth victimization and delinquency: Analysis of Youth Risk Behavior Surveillance Survey data. Cultur Divers Ethnic Minor Psychol. 2010;16(2):123-34. doi: 10.1037/a0018664.

79. Wong SS, Sugimoto-Matsuda JJ, Chang JY, Hishinuma ES. Ethnic differences in risk factors for suicide among American high school students, 2009: The vulnerability of multiracial and Pacific Islander adolescents. Arch Suicide Res. 2012;16(2):159-73. doi: 10.1080/13811118.2012.667334.

80. Rutman S, Park A, Castor M, Taualii M, Forquera R. Urban American Indian and Alaska Native youth: Youth Risk Behavior Survey 1997-2003. Maternal and Child Health Journal. 2008;12 Suppl 1:76-81. doi: 10.1007/s10995-008-0351-3. 
81. Blum RW, Harmon B, Harris L, Bergeisen L, Resnick MD. American Indian--Alaska Native youth health. JAMA. 1992;267(12):1637-44.

82. Borowsky IW, Resnick MD, Ireland M, Blum RW. Suicide attempts among American Indian and Alaska Native youth: Risk and protective factors. Arch Pediatr Adolesc Med. 1999;153(6):573-80.

83. Shaughnessy L, Doshi SR, Jones SE. Attempted suicide and associated health risk behaviors among Native American high school students. J Sch Health. 2004;74(5):177-82.

84. Adair J, Vogt E. Navaho and Zuni veterans: A study of contrasting modes of culture change. American Anthropologist. 1949;51(4):547-61.

85. Norton IM, Manson SM. Research in American Indian and Alaska Native communities: Navigating the cultural universe of values and process. J Consult Clin Psychol. 1996;64(5):85660.

86. Novins DK, Beals J, Roberts RE, Manson SM. Factors associated with suicide ideation among American Indian adolescents: Does culture matter? Suicide Life Threat Behav. 1999;29(4):332-46.

87. Mackin J, Perkins T, Furrer C. The power of protection: A population-based comparison of Native and non-Native youth suicide attempters. Am Indian Alsk Native Ment Health Res. 2012;19(2):20-54. doi: 10.5820/aian.1902.2012.20.

88. Gray JS, Muehlenkamp JJ. Circle of Strength: A case description of culturally integrated suicide prevention. Arch Suicide Res. 2010;14:182-91.

89. Kisch J, Leino EV, Silverman MM. Aspects of suicidal behavior, depression, and treatment in college students: Results from the spring 2000 National College Health Assessment Survey. Suicide Life Threat Behav. 2005;35(1):3-13.

90. Bolton S-L, Elias B, Enns ME. A comparison of the prevalence and risk factors of suicidal ideation and suicide attempts in two American Indian and a general population sample. Transcult Psychiatry. 2014;51(1):3-22. doi: 10.1177/1363461513502574.

91. Harder HG, Rash J, Holyk T, Jovel E, Harder K. Indigenous youth suicide: A systematic review of the literature. Pimatisiwin. 2012;10(1):125-42.

92. Borowsky IW, Ireland M, Resnick MD. Adolescent suicide attempts: Risks and protectors. Pediatrics. 2001;107(3):485-93.

93. Wolitzky-Taylor KB, Ruggiero KJ, McCart MR, Smith DW, Hanson RF, Resnick HS, de Arellano MA, Saunders BE, Kilpatrick DG. Has adolescent suicidality decreased in the United States? Data from two national samples of adolescents interviewed in 1995 and 2005. Journal of Clinical Child \& Adolescent Psychology. 2010;39(1):64-76.

94. Clark TC, Robinson E, Crengle S, Fleming T, Ameratunga S, Denny SJ, Bearinger LH, Sieving RE, Saewyc E. Risk and protective factors for suicide attempt among Indigenous Maori youth in New Zealand: The role of family connection. Journal of Aboriginal Health. 2011;7(1):1631. 
95. Joe S, Baser RS, Neighbors HW, Caldwell CH, Jackson JS. 12-month and lifetime prevalence of suicide attempts among Black adolescents in the National Survey of American Life. J Am Acad Child Adolesc Psychiatry. 2009;48(3):271-82.

96. O'Donnell S, Meyer IH, Schwartz S. Increased risk of suicide attempts among Black and Latino lesbians, gay men, and bisexuals. Am J Public Health. 2011;101(6):1055-9. doi: 10.2105/AJPH.2010.300032.

97. Olshen E, McVeigh KH, Wunsch-Hitzig RA, Rickert VI. Dating violence, sexual assault, and suicide attempts among urban teenagers. Arch Pediatr Adolesc Med. 2007;161(6):539-45. doi: 10.1001/archpedi.161.6.539.

98. Zhao Y, Montoro R, Igartua K, Thombs BD. Suicidal ideation and attempt among adolescents reporting "unsure" sexual identity or heterosexual identity plus same-sex attraction or behavior: Forgotten groups? J Am Acad Child Adolesc Psychiatry. 2010;49(2):104-13. doi: 00004583-201002000-00004.

99. Freedenthal S, Stiffman AR. Suicidal behavior in urban American Indian adolescents: A comparison with reservation youth in a southern state. Suicide Life Threat Behav. 2004;3(2):160-71.

100. van Geel M, Vedder P, Tanilon J. Relationship between peer victimization, cyberbullying, and suicide in children and adolescents: A meta-analysis. JAMA Pediatr. 2014;May 1;168(5):435-42. doi: 10.1001/jamapediatrics.2013.4143.

101. Libby AM, Orton HD, Beals J, Buchwald D, Manson SM. Childhood abuse and later parenting outcomes in two American Indian tribes. Child Abuse Negl. 2008;32(2):195-211. doi: 10.1016/j.chiabu.2007.07.006.

102. Johnson JG, Cohen P, Gould MS, Kasen S, Brown JM, Brook JS. Childhood adversities, interpersonal difficulties, and risk for suicide attempts during late adolescence and early adulthood. Arch Gen Psychiatry. 2002;59:741-9. doi: 10.1001/archpsyc.59.8.741.

103. McLaughlin J, O'Carroll RE, O'Connor RC. Intimate partner abuse and suicidality: A systematic review. Clin Psychol Rev. 2012;32(8):677-89. doi: 10.1016/j.cpr.2012.08.002.

104. Swahn MH, Simon TR, Hertz MF, Arias I, Bossarte RM, Ross JG, Gross LA, lachan R, Hamburger ME. Linking dating violence, peer violence, and suicidal behaviors among high-risk youth. Am J Prev Med. 2008;34(1):30-8. doi: 10.1016/j.amepre.2007.09.020.

105. For the Cedar Project Partnership, Pearce ME, Christian WM, Patterson K, Norris K, Moniruzzaman A, Craib KJP, Schecter MT, Spittal PM. The Cedar Project: Historical trauma, sexual abuse and HIV risk among young Aboriginal people who use injection and non-injection drugs in two Canadian cities. Soc Sci Med. 2008;66:2185-94.

106. Wilson KG, Stelzer J, Bergman JN, Kral MJ, Inayatullah M, Elliott CA. Problem solving, stress, and coping in adolescent suicide attempts. Suicide Life Threat Behav. 1995;25(2):24152.

107. Brent DA, Perper JA, Moritz G, Baugher M, Schweers J, Roth C. Firearms and adolescent suicide: A community case-control study. AJDC. 1993;147:1066-71. 
108. Brent DA, Baugher M, Bridge J, Chen T, Chiappetta L. Age- and sex-related risk factors for adolescent suicide. J Am Acad Child Adolesc Psychiatry. 1999;38(12):1497-505.

109. Kapusta ND, Etzersdorfer E, Krall C, Sonneck G. Firearm legislation reform in the European Union: Impact on firearm availability, firearm suicide and homicide rates in Austria. $\mathrm{Br}$ J Psychiatry. 2007;191:253-7. doi: 10.1192/bjp.bp.106.032862.

110. Bridge JA, Goldstein TR, Brent DA. Adolescent suicide and suicidal behavior. J Child Psychol Psychiatry. 2006;47(3/4):372-94.

111. Anglemyer A, Horvath T, Rutherford G. The accessibility of firearms and risk for suicide and homicide victimization among household members: A systematic review and meta-analysis. Annals of Internal Medicine. 2014;160(2):101-10. doi: 10.7326/M13-1301.

112. Pettingell SL, Bearinger LH, Skay CL, Resnick MD, Potthoff SJ, Eichhorn J. Protecting urban American Indian young people from suicide. Am J Health Behav. 2008;32(5):465-76. doi: 10.5555/ajhb.2008.32.5.465.

113. Yoder KA, Whitbeck LB, Hoyt DR, LaFromboise T. Suicidal ideation among American Indian youths. Arch Suicide Res. 2006;10(2):177-90. doi: 10.1080/13811110600558240.

114. Chino M, Fullerton-Gleason L. Understanding suicide attempts among American Indian adolescents in New Mexico: Modifiable factors related to risk and resiliency. Ethn Dis. 2006;16(2):435-42.

115. Brent DA, Perper JA, Moritz G, Liotus L, Schweers J, Balach L, Roth C. Familial risk factors for adolescent suicide: A case-control study. Acta Psychiatr Scand. 1994;89:52-8.

116. Abel EL, Kruger ML. Educational attainment and suicide rates in the United States. Psychol Rep. 2005;97(1):25-8.

117. Kumar MB, Walls M, Janz T, Hutchinson P, Turner T, Graham C. Suicidal ideation among Métis adult men and women--associated risk and protective factors: Findings from a nationally representative survey. Int J Circumpolar Health. 2012;Aug 6(71):18829. doi: 10.3402/ijch.v71i0.18829.

118. Whitley E, Gunnell D, Dorling D, Smith GD. Ecological study of social fragmentation, poverty, and suicide. BMJ. 1999;319(7216):1034-7.

119. Young TJ. Suicide and homicide among Native Americans: Anomie or social learning? Psychol Rep. 1991;68(3):1137-8.

120. LaFromboise TD, Medoff L, Lee CC, Harris A. Psychosocial and cultural correlates of suicidal ideation among American Indian early adolescents on a Northern Plains reservation. Research in Human Development. 2007;4(1 \& 2):119-43. doi: 10.1080/15427600701481020.

121. LeMaster PL, Beals J, Novins DK, Manson SM. The prevalence of suicidal behaviors among Northern Plains American Indians. Suicide Life Threat Behav. 2004;34(3):242-54. doi: 10.1521/suli.34.3.242.42780. 
122. Luke JN, Anderson IP, Gee GJ, Thorpe R, Rowley KG, Reilly RE, Thorpe A, Stewart PJ. Suicide ideation and attempt in a community cohort of urban Aboriginal youth: A cross-sectional study. Crisis. 2013:1-11. doi: 10.1027/0227-5910/a000187.

123. Saluja G, lachan R, Scheidt PC, Overpeck MD, Sun W, Giedd JN. Prevalence of and risk factors for depressive symptoms among young adolescents. Arch Pediatr Adolesc Med. 2004;158(8):760-5. doi: 10.1001/archpedi.158.8.760.

124. Grossman DC, Milligan M, Deyo RA. Risk factors for suicide attempts among Navajo adolescents. Am J Public Health. 1991;81(7):870-4.

125. Dube SR, Anda RF, Felitti VJ, Chapman DP, Williamson DF, Giles WH. Childhood abuse, household dysfunction, and the risk of attempted suicide throughout the life span: Findings from the Adverse Childhood Experiences Study. JAMA. 2001;286(24):3089-96.

126. Chapman DP, Whitfield CL, Felitti VJ, Dube SR, Edwards VJ, Anda RF. Adverse childhood experiences and the risk of depressive disorders in adulthood. Journal of Affective Disorders. 2004;82:217-25.

127. Whitefield CL, Anda RF, Dube SR, Felitti VJ. Violent childhood experiences and the risk of intimate partner violence in adults. J Interpers Violence. 2003;18(2):166-85.

128. Anda RF, Brown DW, Felitti VJ, Dube SR, Giles WH. Adverse childhood experiences and prescription drug use in a cohort study of adult HMO patients. BMC Public Health. 2008;8(198). doi: 10.1186/1471-2458-8-198.

129. Dube SR, Miller JW, Brown DW, Giles WH, Felitti VJ, Dong M, Anda RF. Adverse childhood experiences and the association with ever using alcohol and initiating alcohol use during adolescence. J Adolescent Health. 2006;38:444.e1-e10.

130. Dube SR, Felitti VJ, Dong M, Chapman DP, Giles WH, Anda RF. Childhood abuse, neglect, and household dysfuction and the risk of illicit drug use: The Adverse Childhood Experiences Study. Pediatrics. 2003;111(3):564-72.

131. Dube SR, Anda RF, Felitti VJ, Edwards VJ, Croft JB. Adverse childhood experiences and personal alcohol abuse as an adult. Addict Behav. 2002;27:713-25.

132. Felitti VJ. The relation between adverse childhood experiences and adult health: Turning gold into lead. The Permanente Journal. 2002;6(44-47).

133. Dube SR, Felitti VJ, Dong M, Giles WH, Anda RF. The impact of adverse childhood experiences on health problems: Evidence from four birth cohorts dating back to 1900. Prev Med. 2003;37:268-77.

134. Koss MP, Yuan NP, Dightman D, Prince RJ, Polacca M, Sanderson B, Goldman D. Adverse childhood experiences and alcohol dependence among seven Native American tribes. Am J Prev Med. 2003;25(3):238-44.

135. Whitesell NR, Beals J, Mitchell CM, Manson SM, Turner RJ. Childhood exposure to adversity and risk of substance-use disorder in two American Indian populations: The mediational role of early substance-use initiation. J Stud Alcohol Drugs. 2009;70(6):971-81. 
136. Werner EE. Resilience in development. Current Directions in Psychological Science. $1995 ; 4(3): 81-5$.

137. Beals J, Manson SM, Mitchell CM, Spicer P. Cultural specificity and comparison in psychiatric epidemiology: Walking the tightrope in American Indian research. Cult Med Psychiatry. 2003;27(3):259-89.

138. Manson SM, Beals J, Klein SA, Croy CD. Social epidemiology of trauma among 2 American Indian reservation populations. Am J Public Health. 2005;95(5):851-9. doi: 10.2105/AJPH.2004.054171.

139. Breslau N, Kessler RC, Chilcoat HD, Schultz LR, Davis GC, Andreski P. Trauma and posttraumatic stress disorder in the community: The 1996 Detroit area survey of trauma. Arch Gen Psychiatry. 1998;55(7):626-32. doi: 10.1001/archpsyc.55.7.626.

140. Stack S. Suicide: A 15-year review of the sociological literature. Part II: Modernization and social integration perspectives. Suicide Life Threat Behav. 2000;30(2):163-76.

141. Boyd-Ball AJ, Manson SM, Noonan C, Beals J. Traumatic events and alcohol use disorders among American Indian adolescents and young adults. J Trauma Stress. 2006;19(6):937-47. doi: 10.1002/jts.20176.

142. Libby AM, Orton HD, Novins DK, Spicer P, Buchwald D, Beals J, Manson SM, AlSUPERPFP Team. Childhood physical and sexual abuse and subsequent alcohol and drug use disorders in two American Indian tribes. J Stud Alcohol. 2004 65(1):74-83.

143. Whitesell NR, Beals J, Mitchell CM, Keane EM, Spicer P, Truner RJ, AI-SUPERPFP Team. The relationship of cumulative and proximal adversity to onset of substance dependence symptoms in two American Indian communities. Drug Alcohol Depend. 2007;91:279-88.

144. Beals J, Manson SM, Shore JH, Friedman M, Ashcraft M, Fairbank JA, Schlenger WE. The prevalence of posttraumatic stress disorder among American Indian Vietnam veterans: Disparities and context. J Trauma Stress. 2002;15(2):89-97. doi: 10.1023/A:1014894506325.

145. Krieger N. Discrimination and health. In: Berkman LF, Kawachi I, editors. Social Epidemiology. Oxford: Oxford University Press; 2000.

146. Krieger N. Does racism harm health? Did child abuse exist before 1962? On explicit questions, critical science, and current controversies: An ecosocial perspective. Am J Public Health. 2003;93(2):194-9.

147. Kaholokula JK, Iwane MK, Nacapoy AH. Effects of perceived racism and acculturation on hypertension in Native Hawaiians. Hawai'i Medical Journal. 2010;69(Supplement 2):11-5.

148. Williams DR, Mohammed SA. Discrimination and racial disparities in health: Evidence and needed research. Journal of Behavioral Medicine. 2009;32(1):20-47.

149. Thoits PA. Stress and health: Major findings and policy implications. J Health Soc Behav. 2010;51(Suppl):S41-53. doi: 10.1177/0022146510383499. 
150. Williams DR, Neighbors HW, Jackson JS. Racial/ethnic discrimination and health: Findings from community studies. Am J Public Health. 2003;93:200-8.

151. Borrell LN, Jacobs Jr DR, Williams DR, Pletcher MJ, Houston TK, Kiefe CI. Self-reported racial discrimination and substance use in the Coronary Artery Risk Development in Adults Study. Am J Epidemiol. 2007;166(9):1068-79. doi: 10.1093/aje/kwm180.

152. Harris R, Tobias M, Jeffreys M, Waldegrave K, Karlsen S, Nazroo J. Racism and health: The relationship between experience of racial discrimination and health in New Zealand. Soc Sci Med. 2006;63:1428-41.

153. Paradies $\mathrm{Y}$. A systematic review of empirical research on self-reported racism and health. Ethnicity and Health. 2006;35:888-901.

154. Pascoe EA, Smart Richman L. Perceived discrimination and health: A meta-analytic review. Psychol Bull. 2009;135(4):531-54. doi: 10.1037/a0016059.

155. Schultz AJ, Gravlee C, Williams DR, Israel BA, Mentz G, Rowe Z. Discrimination, symptoms of depression, and self-rated health among African American women in Detroit: Results from a longitudinal analysis. Am J Public Health. 2006;96(7):1265-70.

156. Noh S, Kaspar V, Wickrama KAS. Overt and subtle racial discrimination and mental health: preliminary findings for Korean immigrants. Am J Public Health. 2007;97(1269-1274).

157. Paradies YC, Cunningham J. The DRUID study: Racism and self-assessed health status in an indigenous population. BMC Public Health. 2012;12(131). doi: 10.1186/1471-2458-12-131.

158. Paradies YC, Cunningham J. The DRUID study: Exploring mediating pathways between racism and depressive symptoms among indigenous Australians. Soc Psychiatry Psychiatr Epidemiol. 2012;47(2):165-73. doi: 10.1007/s00127-010-0332-x.

159. Perry BL, Harp KLH, Oser CB. Racial and gender discrimination in the stress process: Implications for African American women's health and well-being. Sociol Perspect. 2013;56(1):25-48.

160. Pieterse AL, Todd NR, Neville HA, Carter RT. Perceived racism and mental health among Black American adults: A meta-analytic review. J Couns Psychol. 2012;59(1):1-9. doi: 10.1037/a0026208.

161. Hwang WC, Goto S. The impact of perceived racial discrimination on the mental health of Asian American and Latino college students. Cultur Divers Ethnic Minor Psychol. 2008 14(4):326-35. doi: 10.1037/1099-9809.14.4.326.

162. Hunte HER, Barry AE. Perceived discrimination and DSM-IV-based alcohol and illicit drug use disorders. Am J Public Health. 2012;102(12):e111-e7. doi: 10.2105/AJPH.2012.300780.

163. Hunte HER, King K, Hicken M, Lee H, Lewis TT. Interpersonal discrimination and depressive symptomatology: Examination of several personality-related characteristics as potential confounders in a racial/ethnic heterogeneous adult sample. BMC Public Health. 2013;13(1084). doi: 10.1186/1471-2458-13-1084. 
164. Gomez J, Miranda R, Polanco L. Acculturative stress, perceived discrimination, and vulnerability to suicide attempts among emerging adults. J Youth Adolesc. 2011;40(11):146576. doi: 10.1007/s10964-011-9688-9.

165. Lamis DA, Kaslow NJ. Mediators of the daily hassles-suicidal ideation link in African American women. Suicide Life Threat Behav 2014;44(3):233-45. doi: 10.1111/sltb.12099.

166. Walker RL, Salami TK, Carter SE, Flowers K. Perceived racism and suicide ideation: Mediating role of depression but moderating role of religiosity among African American adults. Suicide Life Threat Behav. 2014;44(5):548-59. doi: 10.1111/sltb.12089.

167. Brody GH, Kogan SM, Chen YF. Perceived discrimination and longitudinal increases in adolescent substance use: Gender differences and mediational pathways. Am J Public Health. 2012;102(5):1006-11. doi: 10.2105/AJPH.2011.300588.

168. Basáñez T, Unger JB, Soto D, Crano W, Baezconde-Garbanati L. Perceived discrimination as a risk factor for depressive symptoms and substance use among Hispanic adolescents in Los Angeles. Ethn Health. 2013;18(3):244-61. doi:

10.1080/13557858.2012.713093.

169. Moghaddam JF, Momper SL, Fong T. Discrimination and participation in traditional healing for American Indians and Alaska Natives. J Community Health. 2013;38(6):1115-23. doi: 10.1007/s10900-013-9721-x.

170. Shavers VL, Fagan P, Jones D, Klein WM, Boyington J, Moten C, Rorie E. The state of research on racial/ethnic discrimination in the receipt of health care. Am J Public Health. 2012;102(5):953-66. doi: 10.2105/AJPH.2012.300773.

171. Burgess DJ, Ding Y, Hargreaves M, van Ryn M, Phelan S. The association between perceived discrimination and underutilization of needed medical and mental health care in a multi-ethnic community sample. J Health Care Poor Underserved. 2008;19(3):894-911. doi: 10.1353/hpu.0.0063.

172. Williams DR. The health of men: Structured inequalities and opportunities. Am J Public Health. 2003;93:724-31.

173. Ziersch A, Gallaher G, Baum F, Bentley M. Racism, social resources and mental health for Aboriginal people living in Adelaide. Australian and New Zealand Journal of Public Health. 2011;35(3):231-7. doi: 10.1111/j.1753-6405.2011.00681.x.

174. Hansen KL, Melhus M, Lund E. Ethnicity, self-reported health, discrimination and socioeconomic status: A study of Sami and non-Sami Norwegian populations. Int J Circumpolar Health. 2010;69(2):111-28.

175. Hansen KL, Melhem NM, Hogmo A, Lund E. Ethnic discrimination and bullying in the Sami and non-Sami populations in Norway: The Saminor Study. Int J Circumpolar Health. 2008;6(1):99-115.

176. May PA. Suicide among American Indian youth: A look at the issues. Children Today. 1987; July-August:22-5. 
177. Whitbeck LB, Hoyt DR, McMorris BJ, Chen X, Stubben JD. Perceived discrimination and early substance abuse among American Indian children. J Health Soc Behav. 2001;42(4):40524.

178. Whitbeck LB, Chen X, Hoyt DR, Adams GW. Discrimination, historical loss and enculturation: Culturally specific risk and resiliency factors for alcohol abuse among American Indians. J Stud Alcohol. 2004;65(4):409-18.

179. Barney DD. Risk and protective factors for depression and health outcomes in American Indian and Alaska Native adolescents. Wicazo SA Review. 2001;Spring:135-50.

180. Whitbeck LB, McMorris BJ, Hoyt DR, Stubben JD, Lafromboise T. Perceived discrimination, traditional practices, and depressive symptoms among American Indians in the upper midwest. J Health Soc Behav. 2002;43(4):400-18.

181. Krieger N, Sidney S. Racial discrimination and blood pressure: The CARDIA Study of young black and white adults. Am J Public Health. 1996;86(10):1370-8.

182. Noh S, Kaspar V. Perceived discrimination and depression: Moderating effects of coping, acculturation, and ethnic support. Am J Public Health. 2003;93(2):232-8.

183. Umana-Taylor AJ, Updegraff KA. Latino adolescents' mental health: Exploring the interrelations among discrimination, ethnic identity, cultural orientation, self-esteem, and depressive symptoms. J Adolesc. 2006;30:549-67.

184. Armitage A. Comparing the Policy of Aboriginal Assimilation: Australia, Canada, and New Zealand. Vancouver: University of British Columbia Press; 1998.

185. Spring J. The American School 1642 - 2000. New York: McGraw-Hill Humanities/Social Sciences/Languages 2001.

186. Shelton BL. Legal and historical basis of Indian health care. In: Dixon M, Rioubideaux Y, editors. Promises to Keep: Health Policy for American Indians and Alaska Natives in the 21st Century. Washington, DC: American Public Health Association; 2001.

187. Duran E, Firehammer J, Gonzalez J. Liberation psychology as the path toward healing cultural soul wounds. Journal of Counseling \& Development. 2008 86(3):288-95.

188. Thornton R. American Indian Holocaust and Survival: A Population History since 1492. Norman, OK: University of Oklahoma Press; 1990.

189. Oetzel J, Duran B. Intimate partner violence in American Indian and/or Alaska Native communities: A social ecological framework of determinants and interventions. Am Indian Alsk Native Ment Health Res. 2004;11(3):49-68.

190. Yellow Horse Brave Heart M. The historical trauma response among Natives and its relationship with substance abuse: A Lakota illustration. J Psychoactive Drugs. 2003;35(1):7-13.

191. Hill DL. Relationship between sense of belonging as connectedness and suicide in American Indians. Arch Psychiatr Nurs. 2009;23(1):65-74. 
192. Krieg A. The experience of collective trauma in Australian Indigenous communities. Australian Psychiatry. 2009;17(Supplement). doi: 10.1080/10398560902938621.

193. Yehuda R, Teicher MH, Seckl JR, Grossman RA, Morris A, Bierer LM. Parental posttraumatic stress disorder as a vulnerability factor for low cortisol trait in offspring of holocaust survivors. Arch Gen Psychiatry. 2007 64(9):1040-8.

194. Yehuda R, Bell A, Bierer LM, Schmeidler J. Maternal, not paternal, PTSD is related to increased risk for PTSD in offspring of Holocaust survivors. J Psychiatr Res. 2008;42(13):110411. doi: 10.1016/j.jpsychires.2008.01.002.

195. Scharf M. Long-term effects of trauma: Psychosocial fiunctioning of the second and third generation of Holocaust survivors. Development and Psychopathology. 2007;19:603-22.

196. Sagi-Schwartz A, Van IJzendoorn MH, Bakermans-Kranenberg MJ. Does intergenerational transmission of trauma skip a generation? No meta-analytic evidence for tertiary traumatization with third generation of Holocaust survivors. Attachment \& Human Development. 2008;10(2):105-21.

197. Kellermann NP. Transmitted Holocaust trauma: Curse or legacy? The aggravating and mitigating factors of Holocaust transmission. Isr J Psychiatry Relat Sci. 2008;45(4):263-70.

198. Kellermann NP. Transmission of Holocaust trauma: An integrative view. Psychiatry. 2001;64(3):256-67.

199. Barel E, Van IJzendoorn MH, Sagi-Schwartz A, Bakermans-Kranenberg MJ. Surviving the Holocaust: A meta-analysis of the long-term sequelae of a genocide. Psychological Bulletin. 2010;136(5):677-98.

200. Whitbeck LB, Adams GW, Hoyt DR, Chen X. Conceptualizing and measuring historical trauma among American Indian people. Am J Community Psychol. 2004;33(3-4):119-30.

201. Canby W, Jr. Status of Indian Tribes in American Law Today. The Wash L Rev. 1987;62(11-22).

202. Piccard A. Death by boarding school: "The last acceptable racism" and the United States' genocide of Native Americans. Gonzaga Law Review. 2013;49(1):137-82.

203. Niezen R. Truth and Indignation: Canada's Truth and Reconciliation Commission on Indian Residential Schools. Toronto: University of Toronto Press; 2013.

204. Jacobs MD. White Mother to a Dark Race: Settler Colonialism, Maternalism, and the Removal of Indigenous Children in the American West and Australia, 1880-1940. Lincoln \& London: University of Nebraska Press; 2009.

205. Yamin AE. Ethnic cleansing and other lies: Combining health and human rights in the search for truth and justice in the former Yugoslavia. In: Mann JM, Gruskin S, Grodin MA, Annas GJ, editors. Health and Human Rights: A Reader. New York: Routledge; 1999.

206. Adams DW. Education For Extinction: American Indians and the Boarding School Experience, 1875-1928. Lawrence, KS: University Press of Kansas; 1995. 
207. Milloy JS. A National Crime: The Canadian Government and the Residential School System: University of Manitoba Press; 1999.

208. Unger S, editor. The Destruction of American Indian Families. New York, NY: Association on American Indian Affairs; 1982.

209. Child BJ. Boarding School Seasons: American Indian Families, 1900-1940. Lincoln, NE: University of Nebraska Press; 1998.

210. Hodge FS. No meaningful apology for American Indian unethical research abuses. Ethics \& Behavior. 2012;22(6):431-44.

211. Meriam L, Brown RA, Cloud HR, Dale EE, Duke E, Edwards HR, McKenzie FA, Mark ML, Ryan Jr WC, Spillman WJ. The problem of Indian Administration. Baltimore, MD: The Institute for Government Research, 1928.

212. Archuleta ML, Child B, Lomawaima KT, editors. Away from Home: American Indian Boarding School Experiences. Phoenix, AZ: Museum of New Mexico; 2005.

213. Haebich A. Broken Circles: Fragmenting Indigenous Families 1800-2000. Fremantle: Fremantle Arts Centre Press; 2001.

214. Chavis B. Off-reservation boarding high schools teachers: How are they perceived by former American Indian students? The Social Science Journal. 1999;36(1):33-45.

215. Szasz MC. Education and the American Indian: The Road to Self-Determination since 1928. Albuquerque, NM: University of New Mexico Press; 2003.

216. Ing NR. The effects of residential schools on Native child-rearing practices. Canadian Journal of Native Education. 1991;18(Supplement ):67-118.

217. United States. Special Subcommittee on Indian Education. 91st Congress. Indian Education: A National Tragedy, a National Challenge: 1969 Report of the Committee on Labor and Public Welfare, United States Senate. Vol. No. 91-501. 1969.

218. Bureau of Indian Education National Directory. Department of the Interior, 2009.

219. Palacios J, Kennedy HP. Reflections of Native American teen mothers. J Obstet Gynecol Neonatal Nurs. 2010;39(4):425-34. doi: 10.1111/j.1552-6909.2010.01149.x.

220. Crazy Bull C, Lindquist C, Gipp DM. An act of sovereignty: Governing tribal higher education. Tribal College Journal of American Indian Higher Education. 2015;26(4). Epub May 2, 2015. doi: http://www.tribalcollegejournal.org/archives/29529.

221. DeVoe JF, Darling-Churchill KE. Status and Trends in the Education of American Indians and Alaska Natives: 2008 (NCES 2008-084). National Center for Education Statistics, Institute of Education Sciences, U.S. Department of Education. Washington, DC., 2008.

222. Guerrero MP. Indian Child Welfare Act of 1978: A response to the threat to Indian culture caused by foster and adoptive placements of Indian children. American Indian Law Review. 1979;7(1):51-77. 
223. Watts S. Voluntary adoptions under the Indian Child Welfare Act of 1978: Balancing the interests of children, families, and tribes. Southern California Law Review. 1989;63(1):213-56.

224. Yablon M. The Indian child welfare act amendments of 2003. Family Law Quarterly. 2004;38(3):689-710.

225. Jaffke CL. The "Existing Indian Family" exception to the Indian Child Welfare Act: The states' attempt to slaughter tribal interests of Indian children. Louisiana Law Review. 2006;66:733-61.

226. Cross T, Simmons D, Alberty K, Black F, Funk K, Trope J, Limb G, Wright V, Freundlich M, McCoy-Roth M, Smith E. Time for Reform: A Matter of Justice for American Indian and Alaskan Native Children. National Indian Child Welfare Association. Philadelphia, PA: The Pew Charitable Trusts; 2007.

227. Wood S, Summers A. Disproportionality Rates for Children of Color in Foster Care. Technical Assistance Bulletin. Reno, Nevada: National Council of Juvenile and Family Court Judges; 2014.

228. Schaverien J. Boarding school syndrome: Broken attachments a hidden trauma. British Journal of Psychotherapy. 2011;27(2):138-55.

229. RHS National Team. First Nations Regional Longitudinal Health Survey (RHS) 2002/03: The Peoples' Report. Ottawa, ON: Assembly of First Nations/First Nations Information Governance Committee, 2007.

230. Kaspar V. The lifetime effect of residential school attendance on Indigenous health status. Am J Public Health. 2013;104(11):2184-90. doi: 10.2105/AJPH.2013.301479.

231. Weaver H, Yellow Horse Brave Heart M. Examining two facets of American Indian identity: Exposure to other cultures and the influence of historical trauma. Journal of Human Behavior in the Social Environment. 1999;2(1/2):19-33.

232. Walls ML, Whitbeck LB. Distress among Indigenous North Americans: Generalized and culturally relevant stressors. Society and Mental Health. 2011;1(2):124-36. doi: $10.1177 / 2156869311414919$.

233. Elias B, Mignone J, Hall M, Hong SP, Hart L, Sareen J. Trauma and suicide behaviour histories among a Canadian indigenous population: An empirical exploration of the potential role of Canada's residential school system. Soc Sci Med 2012;74(10):1560-9. doi: 10.1016/j.socscimed.2012.01.026.

234. Anderson HD. Suicide ideation, depressive symptoms, and out-of-home placement among youth in the U.S. child welfare system. J Clin Child Adolesc Psychol. 2011;40(6):790-6. doi: 10.1080/15374416.2011.614588.

235. Keyes MA, Malone SM, Sharma A, lacono WG, McGue M. Risk of suicide attempt in adopted and nonadopted offspring. Pediatrics. 2013;132(4):639-46. doi: 10.1542/peds.20123251. 
236. Qin P, Mortensen PB, Pedersen CB. Frequent change of residence and risk of attempted and completed suicide among children and adolescents. Arch Gen Psychiatry. 2009;66(6):628-32. doi: 10.1001/archgenpsychiatry.2009.20.

237. Boyle M, Leung E, MacMillan $\mathrm{H}$, Trocmé $\mathrm{N}$, Waechter $\mathrm{R}$, Wekerle $\mathrm{C}$. Suicidality among Aboriginal Youth in the Non-Aboriginal Child Protection Services System. Health and WellBeing in the Aboriginal Policy Research Series: Thompson Educational Publishing, Inc; 2013.

238. Kaspar V. Long-term depression and suicidal ideation outcomes subsequent to emancipation from foster care: Pathways to psychiatric risk in the Métis population. Psychiatry Res. 2014;215(2):347-54. doi: 10.1016/j.psychres.2013.09.003.

239. Tatz C. Aboriginal Suicide Is Different: Aboriginal Youth Suicide in New South Wales, the Australian Capital Territory and New Zealand: Towards a Model of Explanation and Alleviation. Sydney: Centre for Comparative Genocide Studies, 1999.

240. Wexler LM. Inupiat youth suicide and culture loss: Changing community conversations for prevention. Soc Sci Med. 2006;63(11):2938-48. doi: 10.1016/j.socscimed.2006.07.022.

241. Kwan J. From taboo to epidemic: Family violence within aboriginal communities. Glob Soc Welf. 2014:1-8. doi: 10.1007/s40609-014-0003-z.

242. Tousignant M, Sioui N. Resilience and aboriginal communities in crisis: Theory and interventions. International Journal of Indigenous Health. 2009;5(1):43-61.

243. Krystal H. Resilience: Accommodation and recovery. In: Parens H, Blum HP, Akhtar S, editors. The Unbroken Soul: Tragedy, Trauma, and Human Resilience. Lanham, MD: Jason Aronson; 2008.

244. Charles G, DeGagné M. Student-to-student abuse in the Indian residential schools in Canada: Setting the stage for further understanding. Child \& Youth Services. 2013;34(4):34359. doi: 10.1080/0145935X.2013.859903.

245. Jervis LL, Beals J, Croy CD, Klein SA, Manson SM, Al-SUPERPFP Team. Historical consciousness among two American Indian tribes. American Behavioral Scientist. 2006;50(4):526-49.

246. Whitbeck LB, Walls ML, Johnson KD, Morrisseau AD, McDougall CM. Depressed affect and historical loss among North American Indigenous adolescents. Am Indian Alsk Native Ment Health Res. 2009;16(3):16-41.

247. Jervis LL, The Al-SUPERPFP Team. Disillusionment, faith, and cultural traumatization on a Northern Plains reservation. Traumatology. 2009;15(1):11-22.

248. Pokhrel P, Herzog T. Historical trauma and substance use among Native Hawaiian college students. Am J Health Behav. 2014;38(3):420-9. doi: http://dx.doi.org/10.5993/AJHB.38.3.11.

249. Erikson EH. Childhood and tradition in two American Indian tribes. Psychoanal Study Child. 1945;1:319-50. 
250. Cunneen C, Libesman T. Postcolonial trauma: The contemporary removal of Indigenous children and young people from their families in Australia. Australian Journal of Social Issues. 2000;35(2):99-115.

251. Morrissette PJ. The holocaust of First Nation people: Residual effects on parenting and treatment implications. Contemporary Family Therapy. 1994;16(5):381-92.

252. Red Horse J. Traditional American Indian family systems. Fam Syst \& Health. 1997;15:243-50.

253. Trimble JE, Fleming CM, Beauvais F, Jumper-Thurman P. Essential cultural and social strategies for counseling Native American Indians. In: Pedersen PB, Draguns JG, Lonner WJ, Trimble JE, editors. Counseling Across Cultures. 4th ed. Thousand Oaks: SAGE Publications; 1996.

254. Grandbois D. Stigma of mental illness among American Indian and Alaska Native nations: Historical and contemporary perspectives. Issues Ment Health Nurs. 2005;26(10):100124.

255. McShane KE, Hastings PD, Smylie JK, Prince C, Tungasuvvingat Inuit Family Resource Centre. Examining evidence for autonomy and relatedness in urban Inuit parenting. Culture \& Psychology. 2009;15(4):411-31.

256. Goforth S. Traditional parenting skills in contemporary life. Healing Words. 2003;4(1):179.

257. Ball J. Fathering in the shadows: Indigenous fathers and Canadas colonial legacies. The Annals of the American Adademy. 2009;624(1):29-48.

258. Sarche MC, Croy CD, Crow CB, Mitchell CM, Spicer P. Maternal correlates of 2-year-old American Indian children's social-emotional development in a Northern Plains tribe. Infant Ment Health J. 2009;30:321-40. doi: 10.1002/imhj.20217.

259. DiLillo D, Damashek A. Parenting characteristics of women reporting a history of childhood sexual abuse. Child Maltreatment. 2003;8(4):319-33.

260. Noll JG, Trickett PK, Harris WW, Putnam FW. The cumulative burden borne by offspring whose mothers were sexually abused as children. J Interpers Violence. 2009;24 (3):424-49.

261. Saltzberg M. Parenting challenges for women with abuse histories. Journal of Feminist Family Therapy. 2000;12(1):45-58.

262. Banyard VL, Williams LM, Siegel JA. The impact of complex trauma and depression on parenting: An exploration of mediating risk and protective factors. Child Maltreatment. 2003;8(4):334-49.

263. Silviken A, Kvernmo S. Suicide attempts among Indigenous Sami adolescents and majority peers in Arctic Norway: Prevalence and associated risk factors. J Adolesc.

2007;30(4):613-26. doi: 10.1016/j.adolescence.2006.06.004. 
264. Swahn MH, Bossarte RM, Sullivent EE. Age of alcohol use initiation, suicidal behavior, and peer and dating violence victimization and perpetration among high-risk, seventh-grade adolescents. Pediatrics. 2008;121(2):297-305. doi: 10.1542/peds.2006-2348.

265. Tousignant M, Bastien MF, Hamel S. Suicidal attempts and ideations among adolescents and young adults: The contribution of the father's and mother's care and of parental separation. Soc Psychiatry Psychiatr Epidemiol. 1993;28:256-61.

266. Hexel FX. Suicide and the Micronesian family. The Contemporary Pacific. 1989;1(1 \& 2):43-74.

267. Kuhlberg JA, Pena JB, Zayas LH. Familism, parent-adolescent conflict, self-esteem, internalizing behaviors and suicide attempts among adolescent Latinas. Child Psychiatry Hum Dev. 2010;41:425-40.

268. Lim SL, Yeh M, Liang J, Lau AS, K M. Acculturation gap, intergenerational conflict, parenting style, and youth distress in immigrant Chinese American families. Marriage \& Family Review. 2009;45:84-106.

269. Tester FJ, McNicoll P. Isumagijaksaq: Mindful of the state: Social constructions of Inuit suicide. Soc Sci Med. 2004;58(12):2625-36. doi: 10.1016/j.socscimed.2003.09.021.

270. Kellermann NP. Diagnosis of Holocaust survivors and their children. Isr J Psychiatry Relat Sci. 1999;36(1):55-64.

271. Walls ML, Whitbeck LB. The intergenerational effects of relocation policies on Indigenous families. J Fam Issues. 2014;44(3):233-45. doi: 10.1177/0192513X12447178.

272. Ehlers CL, Gizer IR, Gilder DA, Yehuda R. Lifetime history of traumatic events in an American Indian community sample: Heritability and relation to substance dependence, affective disorder, conduct disorder and PTSD. J Psychiatr Res. 2013;47(2):155-61.

273. Stein MB, Jang KL, Taylor S, Vernon PA, Livesley WJ. Genetic and environmental influences on trauma exposure and posttraumatic stress disorder symptoms: A twin study. Am J Psychiatry. 2002;159(10):1675-81.

274. Afifi TO, Asmundson GJ, Taylor S, Jang KL. The role of genes and environment on trauma exposure and posttraumatic stress disorder symptoms: A review of twin studies. Clin Psychol Rev. 2010;30(1):101-12. doi: 10.1016/j.cpr.2009.10.002.

275. Bombay A, Matheson $\mathrm{K}$, Anisman $\mathrm{H}$. The impact of stressors on second generation Indian residential school survivors. Transcult Psychiatry. 2011;48(4):367-91. doi:

10.1177/1363461511410240.

276. Kellermann NPF. Holocaust Trauma: Psychological Effects and Treatment. Bloomington, IN: iUniverse; 2009.

277. Kellermann NP. Epigenetic transmission of holocaust trauma: Can nightmares be inherited? Isr J Psychiatry Relat Sci. 2013;50(1):33-9. 
278. Hyde-Nolan ME, Juliao T. Theoretical basis for family violence. In: Fife RS, Schrager S, editors. Family Violence: What Health Care Providers Need To Know. London: Jones and Bartlett Learning, LLC; 2012.

279. Bandura A. Social Learning Theory;1977.

280. Lichtman H. Parental communication of Holocaust experiences and personality characteristics among second-generation survivors. Journal of Clinical Psychology. 1984;40(4):914-24. doi: 10.1002/1097-4679(198407)40:4<914::AIDJCLP2270400408>3.0.CO;2-U.

281. International Handbook of Multigenerational Legacies of Trauma. Danieli Y, editor. New York \& London: Plenum; 1998.

282. Bombay A, Matheson $\mathrm{K}$, Anisman $\mathrm{H}$. Intergenerational trauma: Convergence of multiple processes among First Nations peoples in Canada. Journal of Aboriginal Health. 2009;5(3):647.

283. Paris J. Predispositions, personality traits, and posttraumatic stress disorder. Harv Rev Psychiatry. 2000;8(4):175-83.

284. van der Kolk BA. The body keeps the score: Memory and the evolving psychobiology of posttraumatic stress. Harv Rev Psychiatry. 1994;1(5):253-65.

285. Meaney MJ. Maternal care, gene expression, and the transmission of individual differences in stress reactivity across generations. Annu Rev Neurosci. 2001;24:1161-92.

286. Meaney MJ. Epigenetics and the biological definition of gene $\mathrm{x}$ environment interactions. Child Dev. 2010;81(1):41-79. doi: 10.1111/j.1467-8624.2009.01381.x.

287. Stein RA, Davis DL. Epigenetics: A fascinating field with profound research, clinical, \& public health implications. The American Biology Teacher. 2012 74(4):213-23.

288. Mann JJ, Currier DM. Stress, genetics and epigenetic effects on the neurobiology of suicidal behavior and depression. European Psychiatry. 2010;25:268-71.

289. McGowan PO, Szyf M. The epigenetics of social adversity in early life: Implications for mental health outcomes. Neurobiology of Disease. 2010;39.

290. Thomson P. The impact of trauma on the embryo and fetus: An application of the diathesis-stress model and the neurovulnerability-neurotoxicity model. Journal of Prenatal and Perinatal Psychology and Health. 2004;19(1):9-63.

291. Miller G. The seductive allure of behavioral epigenetics. Science. 2010;329:24-7.

292. Shelton RC. The molecular neurobiology of depression. Psychiatr Clin North Am. 2007;30(1):1-11. doi: 10.1016/j.psc.2006.12.005.

293. Weaver IC, Szyf M, Meaney MJ. From maternal care to gene expression: DNA methylation and the maternal programming of stress responses. Endocr Res. 2002 28(4):699. 
294. Weaver ICG, Cervoni N, Champagne FA, D'Alessio AC, Sharma S, Seckl JR, Dymov S, Szyf M, Meaney MJ. Epigenetic programming by maternal behavior. Nature Neuroscience. 2004;7:847-54. doi: 10.1038/nn1276.

295. Yehuda R, Bierer LM. The relevance of epigenetics to PTSD: Implications for the DSMV. J Trauma Stress. 2009;22(5):427-34. doi: 10.1002/jts.20448.

296. Yehuda R, Bierer LM. Transgenerational transmission of cortisol and PTSD risk. Prog Brain Res. 2008;167(121-35). doi: 10.1016/S0079-6123(07)67009-5.

297. Bierer LM, Bader HN, Daskalakis NP, Lehrner A, Makotkine I, SeckI JR, Yehuda R. Elevation of $11 \beta$-hydroxysteroid dehydrogenase type 2 activity in Holocaust survivor offspring: Evidence for an intergenerational effect of maternal trauma exposure.

Psychoneuroendocrinology. 2014;48:1-10.

298. Yehuda R, Engel SM, Brand SR, Seckl J, Marcus SM, Berkowitz GS. Transgenerational effects of posttraumatic stress disorder in babies of mothers exposed to the World Trade Center attacks during pregnancy. J Clin Endocrinol Metab. 2005;90(7):4115-8.

299. Oberlander TF, Weinberg J, Papsdorf M, Grunau R, Misri S, Devlin AM. Prenatal exposure to maternal depression, neonatal methylation of human glucocorticoid receptor gene (NR3C1) and infant cortisol stress responses. Epigenetics. 2008;3(2):97-106.

300. Uddin M, Aiello AE, Wildman DE, Koenen KC, Pawelec G, de Los Santos R, Goldmann $\mathrm{E}$, Galea S. Epigenetic and immune function profiles associated with posttraumatic stress disorder. Proc Natl Acad Sci U S A. 2010;107(20):9470-5. doi: 10.1073/pnas.0910794107.

301. Uddin M, Koenen KC, Aiello AE, Wildman DE, de Los Santos R, Galea S. Epigenetic and inflammatory marker profiles associated with depression in a community-based epidemiologic sample. Psychol Med. 2011;41(5):997-1007. doi: 10.1017/S0033291710001674.

302. Bale TL, Baram TZ, Brown AS, Goldstein JM, Insel TR, McCarthy MM, Nemeroff CB, Reyes TM, Simerly RB, Susser ES, Nestler EJ. Early life programming and neurodevelopmental disorders. Biol Psychiatry. 2010;68(4):314-9. doi: 10.1016/j.biopsych.2010.05.028.

303. Brockie TN, Heinzelmann M, Gill J. A framework to examine the role of epigenetics in health disparities among Native Americans. Nursing Research and Practice. 2013;2013. doi: $10.1155 / 2013 / 410395$.

304. Weaver ICG, Champagne FA, Brown SE, Dymov S, Sharma S, Meaney MJ, Szyf M. Reversal of maternal programming of stress responses in adult offspring through methyl supplementation: Altering epigenetic marking later in life The Journal of Neuroscience. 2005;25(47):11045-54. doi: 10.1523/JNEUROSCI.3652-05.2005.

305. Mello MM, Wolf LE. The Havasupai Indian tribe case - Lessons for research involving stored biological samples. New England Journal of Medicine. 2010;263:204-7. doi: 10.1056/NEJMp1005203.

306. Mohatt GV, Thomas LR. I wonder, why would you do it that way? Ethical dilemmas in doing participatory research with Alaska Native communities. In: Trimble JE, Fisher CB, editors. 
The Handbook of Ethical Research with Ethnocultural Populations \& Communities. Thousand Oaks, CA: Sage; 2005.

307. Middlebrook DL, LeMaster PL, Beals J, Novins DK, Manson SM. Suicide prevention in American Indian and Alaska Native communities: A critical review of programs. Suicide Life Threat Behav. 2001;31 Suppl:132-49.

308. Hemenway D. While We Were Sleeping: Success Stories in Injury and Violence Prevention. Berkeley and Los Angeles: University of California Press; 2009.

309. Rockett IRH. Counting suicides and making suicide count as a public health problem. Crisis. 2010;31(5):227-30.

310. Garroutte EM, Goldberg J, Beals J, Herrell R, Manson SM. Spirituality and attempted suicide among American Indians. Soc Sci Med. 2003;56(7):1571-9. doi: 10.1016/S02779536(02)00157-0.

311. Gould MS, Greenberg T, Velting DM, Shaffer D. Youth suicide risk and preventive interventions: A review of the past 10 years. Journal of the American Academy of Child \& Adolescent Psychiatry. 2003;42(4):386-405.

312. Lizardi D, Gearing RE. Religion and suicide: Buddhism, Native American and African religions, atheism, agnosticism. J Relig Health. 2010;49:377-84.

313. Kleiman EM, Liu RT. Social support as a protective factor in suicide: Findings from two nationally representative samples. J Affect Disord. 2013;150(2):540-5. doi: 10.1016/j.jad.2013.01.033.

314. Kleiman EM, Riskind JH, Schaefer KE. Social support and positive events as suicide resiliency factors: Examination of synergistic buffering effects. Arch Suicide Res. 2014;18(2):144-55. doi: 10.1080/13811118.2013.826155.

315. deMan AF, Becerril Gutierrez BI. The relationship between level of self-esteem and suicidal ideation with stability of self-esteem as moderator. Canadian Journal of Behavioural Science. 2002;34(4):235-8.

316. FrankI VE. Man's Search for Meaning. New York: Washington Square Press; 1984.

317. Davis TL, Kerr BA, Kurpius SER. Meaning, purpose, and religiosity in at-risk youth: The relationship between anxiety and spirituality. Journal of Psychology \& Theology.

2003;31(4):356-65.

318. Park CL. Religiousness/spirituality and health: A meaning systems perspective. Journal of Behavioral Medicine. 2007;30(4):319-28.

319. Wong YJ, Rew L, Slaikeu KD. A systematic review of recent research on adolescent religiosity/spirituality and mental health. Issues in Mental Health Nursing. 2006;27(2):161-83 doi: $10.1080 / 01612840500436941$. 
320. Dew RE, Daniel SS, Armstrong TD, Goldston DB, Triplett MF, Koenig HG.

Religion/spirituality and adolescent psychiatric symptoms: A review. Child Psychiatry and Human Development. 2008;39(4):381-98.

321. Bullock M, Nadeau L, Renaud J. Spirituality and religion in youth suicide attempters' trajectories of mental health service utilization: The year before a suicide attempt. J Can Acad Child Adolesc Psychiatry. 2012;21(3):186-93.

322. Pescosolido BA, Georgianna S. Durkheim, suicide, and religion: Toward a network theory of suicide. Am Sociol Rev. 1989;54(1):33-48.

323. Stack $\mathrm{S}$. The effect of religious commitment on suicide: A cross-national analysis. J Health Soc Behav. 1983;24(4):362-74.

324. Davidson CL, Wingate LR. Racial disparities in risk and protective factors for suicide. Journal of Black Psychology. 2011:1-18. doi: 10.1177/0095798410397543.

325. Taliaferro LA, Rienzo BA, Pigg RM, Miller MD, Dodd VJ. Spiritual well-being and suicidal ideation among college students. Journal of American College Health. 2009;58(1):83-90. doi: 10.3200/JACH.58.1.83-90.

326. Hallett D, Chandler MJ, Lalonde CE. Aboriginal language knowledge and youth suicide. Cognitive Development. 2007;22:392-9. doi: 10.1016/j.cogdev.2007.02.001.

327. O'Donnell L, O'Donnell C, Wardlaw DM, Stueve A. Risk and resiliency factors influencing suicidality among urban African American and Latino youth. Am J Community Psychol. 2004;33(1/2):37-49.

328. Manson SM, Beals J, Dick RW, Duclos C. Risk factors for suicide among Indian adolescents at a boarding school. Public Health Rep. 1989;104(6):609-14.

329. Taylor SE, Welch WT, Kim HS, Sherman DK. Cultural differences in the impact of social support on psychological and biological stress responses. Psychological Science. 2007;18:8317.

330. Oetzel J, Duran B, Jiang Y, Lucero J. Social support and social undermining as correlates for alcohol, drug, and mental disorders in American Indian women presenting for primary care at an Indian Health Service hospital. J Health Commun. 2007;12(2):187-206.

331. Higgins PG, Dicharry EK. Measurement issues addressing social support with Navajo women. West J Nurs Res. 1991;13(2):242-55.

332. Twenge JM, Crocker J. Race and self-esteem: Meta-analyses comparing Whites, Blacks, Hispanics, Asians, and American Indians and comment on Gray-Little and Hafdahl. Psychol Bull. 2002 128(3):371-408.

333. Hobfoll SE, Jackson A, Hobfoll I. The impact of communal-mastery versus self-mastery on emotional outcomes during stressful conditions: A prospective study of Native American women. Am J Community Psychol. 2002;30(6):853-71. 
334. Luthar SS, Cicchetti D, Becker B. The construct of resilience: A critical evaluation and guidelines for future work. Child Dev. 2000;71(3):543-62.

335. Davydov DM, Stewart R, Ritchie K, Chaudieu I. Resilience and mental health. Clin Psychol Rev. 2010;30(5):479-95. doi: 10.1016/j.cpr.2010.03.003.

336. LaFromboise TD, Hoyt DR, Oliver L, Whitbeck LB. Family, community, and school influences on resilience among American Indian adolescents in the upper midwest. J Community Psychol. 2006;34(2):193-209. doi: 10.1002/jcop.20090.

337. Fleming J, Ledogar RJ. Resilience, an evolving concept: A review of literature relevant to aboriginal research. Pimatisiwin. 2008;6(2):7-23.

338. Kirmayer LJ, Sehdev M, Whitley R, Dandeneau SF, Isaac C. Community resilience: Models, metaphors and measures. International Journal of Indigenous Health. 2009;5(1).

339. Johnson J, Wood AM, Gooding G, Taylor PJ, Tarrier N. Resilience to suicidality: The buffering hypothesis. Clin Psychol Rev. 2011;31(4):563-91. doi: 10.1016/j.cpr.2010.12.007.

340. Roy A, Carli V, Sarchiapone M. Resilience mitigates the suicide risk associated with childhood trauma. Journal of Affective Disorders. 2011;133(3):591-4. doi:

10.1016/j.jad.2011.05.006.

341. Goins RT, Gregg JJ, Fiske A. Psychometric properties of the Connor-Davidson resilience scale with older American Indians: The Native elder care study. Research on Aging. 2013;35(2):123-43 doi: 10.1177/0164027511431989.

342. Stiffman AR, Brown E, Freedenthal S, House L, Ostmann E, Yu MS. American Indian youth: Personal, familial, and environmental strengths. Journal of Child and Family Studies. 2007;16(3):331-46. doi: 10.1007/s10826-006-9089-y.

343. Zahradnik M, Stewart SH, O'Connor RM, Stevens D, Ungar M, Wekerle C. Resilience moderates the relationship between exposure to violence and posttraumatic reexperiencing in Mi'kmaq youth. International Journal of Mental Health and Addiction. 2010;8(2):408-20.

344. Stumblingbear-Riddle G, Romans JS. Resilience among urban American Indian adolescents: Exploration into the role of culture, self-esteem, subjective well-being, and social support. Am Indian Alsk Native Ment Health Res. 2012;19(2):1-19. doi:

10.5820/aian.1902.2012.1.

345. Fleming J, Ledogar RJ. Resilience and indigenous spirituality: A literature review. Pimatisiwin. 2008;6(2):47-64.

346. Goin L, Mill JE. Resilience: A health promoting strategy for aboriginal women following family suicide. Pimatisiwin. 2013;11(3):485-99.

347. Andersson N, Ledogar RJ. The CIET Aboriginal youth resilience studies: 14 years of capacity building and methods development in Canada. Pimatisiwin. 2008;6(2):65-88. 
348. Andersson N, Shea B, Amaratunga C, McGuire P, Sioui G. Rebuilding from resilience: Research framework for a randomized controlled trial of community-led interventions to prevent domestic violence in Aboriginal communities. Pimatisiwin. 2010;8(2):61-88.

349. Southwick SM, Ozbay F, Mayes LC. Psychological and biological factors associated with resilience to stress and trauma. In: Henri Parens HPB, Salman Akhtar, editor. The Unbroken Soul: Tragedy, Trauma, and Resilience. Lanham. MD: Jason Aronson; 2008.

350. Bowlby J. A Secure Base: Clinical Applications Of Attachment Theory. London: Routledge; 1988.

351. Karatoreos IN, McEwen BS. Annual Research Review: The neurobiology and physiology of resilience and adaptation across the life course. J Child Psychol Psychiatry. 2013;54(4):33747. doi: 10.1111/jcpp.12054.

352. Kim-Cohen J, Gold AL. Measured gene-environment interactions and mechanisms promoting resilient development. Current Directions in Psychological Science. 2009;18(3):13842. doi: 10.1111/j.1467-8721.2009.01624.x

353. Matija Svetina M. Resilience in the context of Erikson's theory of human development. Current Psychology. 2014;33(3):393-404. doi: 10.1007/s12144-014-9218-5.

354. Foley D. Indigenous epistemology and indigenous standpoint theory. Social Alternatives. 2003;22(1):44-52.

355. Chandler M. Surviving time: The persistence of identity in this culture and that. Culture \& Psychology. 2000;6(2):209-31.

356. Chandler M, Proulx T. Changing selves in changing worlds: Youth suicide on the faultlines of colliding cultures. Arch Suicide Res. 2006;10(2):125-40.

357. Varenne H. Collective representations in American anthropological conversations: Individual and culture. Current Anthropology. 1984;25(3):281-300.

358. Douglas M. Purity and Danger: An Analysis of the Concept of Pollution and Taboo. New York: Routledge and Keegan Paul; 1966.

359. Demerath NJ. Crossing the Gods: World Religions and Worldly Politics. New Brunswick, NJ: Rutgers University Press; 2001.

360. Geertz C. The Interpretation of Culture: Selected Essays. Books B, editor. New York; 1973.

361. Nagel J, Snipp CM. Ethnic reorganization: American Indian social, economic, political, and cultural strategies for survival. Ethnic \& Racial Studies. 1993;16(2):203-35.

362. Said EW. Culture and Imperialism. New York: Knopf; 1993.

363. Zimmerman MA, Ramirez-Valles J, Washienko KM, Walter B, Dyer S. The development of a measure of enculturation for Native American youth. Am J Community Psychol.

1996;24(2):295-310. 
364. Torres Stone RA, Whitbeck LB, Chen X, Johnson K, Olson DM. Traditional practices, traditional spirituality, and alcohol cessation among American Indians. J Stud Alcohol.

2006;67:236-44.

365. Galliher RV, Jones MD, Dahl A. Concurrent and longitudinal effects of ethnic identity and experiences of discrimination on psychosocial adjustment of Navajo adolescents. Dev Psychol. 2011;47(2):500-26.

366. Rieckmann TR, Wadsworth ME, Deyhle D. Cultural identity, explanatory style, and depression in Navajo adolescents. Cultur Divers Ethnic Minor Psychol. 2004;10(4):365-82. doi: 10.1037/1099-9809.10.4.365.

367. Bombay A, Matheson K, Anisman H. Decomposing identity: Differential relationships between several aspects of ethnic identity and the negative effects of perceived discrimination among First Nations adults in Canada. Cultur Divers Ethnic Minor Psychol. 2010;16(4):507-16. doi: 10.1037/a0021373.

368. Moran JR, Fleming CM, Somervell P, Manson SM. Measuring bicultural ethnic identity among American Indian adolescents: A factor analytic study. Journal of Adolescent Research. 1999;14(4):405-26.

369. Kenyon DB, Carter JS. Ethnic identity, sense of community, and psychological wellbeing among northern plains American Indian youth. Journal of Community Psychology. 2011;39(1):1-9. doi: 10.1002/jcop.20412.

370. Erikson EH. Identity, Youth and Crisis. New York: W. W. Norton Company; 1968.

371. Markstrom CA. Identity formation of American Indian adolescents: Local, national, and global considerations. Journal of Research on Adolescence. 2011;21(2):519-35. doi:

10.1111/j.1532-7795.2010.00690.x.

372. Rivas-Drake D, Seaton EK, Markstrom C, Quintana S, Syed M, Lee RM, Schwartz SJ, Umaña-Taylor AJ, French S, Yip T, and Ethnic and Racial Identity in the 21st Century Study Group. Ethnic and racial identity in adolescence: Implications for psychosocial, academic, and health outcomes. Child Dev. 2014;85:40-57. doi: 10.1111/cdev.12200.

373. Erikson EH. Identity and the Life Cycle. New York: W W Norton \& Company, Inc; 1980.

374. Cheah CSL, Nelson LJ. The role of acculturation in the emerging adulthood of aboriginal college students. International Journal of Behavioral Development. 2004;28(6):495-507 doi: 10.1080/01650250444000135.

375. Spencer MB, Markstrom-Adams C. Identity processes among racial and ethnic minority children in America. Child Dev. 1990;61:290-310. doi: 10.1111/j.1467-8624.1990.tb02780.x.

376. Lazarus RS, Folkman S. The coping process: An alternative to traditional formulas. Stress, Appraisal, and Coping. New York: Springer Publishing Co; 1984. p. 141.

377. Stewart SH, Sherry SB, Comeau MN, Mushquash CJ, Collins P, Van Wilgenburg H. Hopelessness and excessive drinking among Aboriginal adolescents: The mediating roles of 
depressive symptoms and drinking to cope. Depression Research and Treatment. 2011;2010. doi: $10.1155 / 2011 / 970169$.

378. Pittman CT. Getting mad but ending up sad: The mental health consequences for African Americans using anger to cope with racism. Journal of Black Studies. 2011. doi: 10.1177/0021934711401737.

379. Brown SP, Westbrook RA, Challagalla G. Good cope, bad cope: Adaptive and maladaptive coping strategies following a critical negative work event. J Appl Psychol. 2005;90(4):792-8.

380. Aldwin CM, Revenson TA. Does coping help? A reexamination of the relation between coping and mental health. J Pers Soc Psychol. 1987;53(2):337-48.

381. Wadsworth ME, Rieckmann T, Benson MA, Compas BE. Coping and responses to stress in Navajo adolescents: Psychometric properties of the Responses to Stress Questionnaire. Journal of Community Psychology. 2004;32(4):391-411.

382. LaVeist TA, Thorpe RJ, Pierre G, Mance GA, Williams DR. The relationships among vigilant coping style, race, and depression. Journal of Social Issues. 2014;70:241-55. doi: 10.1111/josi.12058.

383. Wolsko C, Lardon C, Mohatt GV, E O. Stress, coping, and well-being among the Yup'ik of the Yukon-Kuskokwim Delta: The role of enculturation and acculturation. Int $\mathrm{J}$ Circumpolar Health. 2007;66(1):51-61.

384. Iwasaki Y, Bartlett J, O'Neil J. Coping with stress among Aboriginal women and men with diabetes in Winnepeg, Canada. Soc Sci Med. 2005;60:977-88.

385. McCormick R, Wong PTP. Adjustment and coping in Aboriginal people. Handbook of Multicultural Perspectives on Stress and Coping. 2006;4:515-31.

386. McNulty Eitle T, Eitle D. Race, coping strategies, and substance use behaviors: A preliminary analysis examining white and American Indian adolescents. Subst Use Misuse. 2014;49(3):315-25. doi: 10.3109/10826084.2013.832329.

387. Latham AE, Prigerson HG. Suicidality and bereavement: Complicated grief as psychiatric disorder presenting greatest risk for suicidality. Suicide Life Threat Behav. 2004;34:350-62. doi: 10.1521/suli.34.4.350.53737.

388. Muehlenkamp JJ, Marrone S, Gray JS. A college suicide prevention model for American Indian students. Professional Psychology: Research and Practice. 2009;40(2):134-40.

389. Olson LM, Wahab S. American Indians and suicide: A neglected area of research. Trauma Violence \& Abuse. 2006;7(1):19-33. . doi: 10.1177/1524838005283005.

390. Eaton DK, Foti K, Brener ND, Crosby AE, Flores G, Kann L. Associations between risk behaviors and suicidal ideation and suicide attempts: Do racial/ethnic variations in associations account for increased risk of suicidal behaviors among Hispanic/Latina 9th- to 12th-grade female students? Arch Suicide Res. 2011;15:113-26. doi: 10.1080/13811118.2011.565268. 
391. Reyes JC, Robles RR, Colón HM, Negrón JL, Matos TD, Calderón JM. Polydrug use and attempted suicide among Hispanic adolescents in Puerto Rico. Arch Suicide Res. 2011;15(2):151-9. doi: 10.1080/13811118.2011.565274.

392. Eaton DK, Lowry R, Brener ND, Galuska DA, Crosby AE. Associations of body mass index and perceived weight with suicide ideation and suicide attempts among US high school students. Arch Pediatr Adolesc Med. 2005;159:513-9.

393. Klomek AB, Marrocco F, M K, Schonfield IS, Gould MS. Bullying, depression, and suicidality in adolescents. Journal of the American Academy of Child \& Adolescent Psychiatry. 2007;46(1):40-9. doi: 10.1097/01.chi.0000242237.84925.18.

394. Goldston DB, Molock SD, Whitbeck LB, Murakami JL, Zayas LH, Hall GC. Cultural considerations in adolescent suicide prevention and psychosocial treatment. American Psychologist. 2008;63(1):14-31. doi: 10.1037/0003-066X.63.1.14.

395. Eaton DK, Kann L, Kinchen S, Ross J, Hawkins J, Harris WA, Lowry R, McManus T, Chyen D, Shanklin S, Lim C, Grunbaum JA, Wechsler H. Youth Risk Behavior Surveillance-United States, 2005. Morbidity and Mortality Weekly Report Surveillance Summary. 2006;55(5):1-108.

396. SAS Institute Inc. SAS/STAT® 9.2 User's Guide. Cary, NC: SAS Institute Inc.; 2008.

397. CDC. Methodology of the Youth Risk Behavior Surveillance System. MMWR. 2004;53(RR-12):1-14.

398. Costello EJ, Farmer EM, Angold A, Burns BJ, Erkanli A. Psychiatric disorders among American Indian and White youth in Appalachia: The Great Smoky Mountains Study. Am J Public Health. 1997;87(5):827-32.

399. Whitbeck LB, Yu M, Johnson KD, Hoyt DR, Walls ML. Diagnostic prevalence rates from early to mid-adolescence among Indigenous adolescents: First results from a longitudinal study. Journal of the American Academy of Child \& Adolescent Psychiatry. 2008;Aug; 47(8):890-900. doi: $10.1097 / \mathrm{CHI} .0 \mathrm{b013e3181799609.}$

400. Rao U. Links between depression and substance abuse in adolescents: Neurobiological mechanisms. Am J Prev Med. 2006;31(6 Suppl 1):S161-S74.

401. Corte C, Stein KF. Eating disorders and substance use: An examination of behavioral associations. Eating Behaviors. 2000;1(2):173-89.

402. Montana Office of Public Instruction. Montana statewide dropout and graduate report: 2008-2009 school year. 2010.

403. Singh GK, Kogan MD. Widening socioeconomic disparities in US childhood mortality, 1969-2000. Am J Public Health. 2007;97(9):1658-65. doi: 10.2105/AJPH.2006.087320.

404. Katz LY, Au W, Singal D, Brownell M, Roos N, Martens PJ, Chateau D, Enns MW, Kozyrskyj AL, Sareen J. Suicide and suicide attempts in children and adolescents in the child welfare system. Canadian Medical Association Journal. 2011. doi: 10.1503/cmaj.110749. 
405. Gone JP, Trimble JE. American Indian and Alaska Native mental health: Diverse perspectives on enduring disparities. Annual Review of Clinical Psychology. 2012;8:131-60. doi: 10.1146/annurev-clinpsy-032511-143127.

406. Cohen J. Statistical Power Analysis for the Behavioral Sciences. 2nd ed. Hillsdale, NJ: Lawrence Erlbaum Associates; 1988.

407. O’Nell TD. Disciplined Hearts: History, Identity and Depression in an American Indian Community. Berkeley \& Los Angeles, CA: University of California Press; 1996.

408. May A, Klonsky ED. Validity of suicidality items from the Youth Risk Behavior Survey in a high school sample. Assessment. 2011;18(3):379-81. doi: 10.1177/1073191110374285.

409. American Indian Education Handbook. Sacramento, CA: Superintendent of Public Instruction. California State Department of Education.; 1982.

410. May PA, Van Winkle NW, Williams MB, McFeeley PJ, DeBruyn LM, Serna P. Alcohol and suicide death among American Indians of New Mexico: 1980-1998. Suicide Life Threat Behav. 2002;32(3):240-55.

411. Novins DK, Duclos CW, Martin C, Jewett CS, Manson SM. Utilization of alcohol, drug, and mental health treatment services among American Indian adolescent detainees. J Am Acad Child Adolesc Psychiatry. 1999;38(9):1102-8. doi: 10.1097/00004583-199909000-00013.

412. Manzo K, Tiesman H, Stewart J, Hobbs GR, Knox SS. A comparison of risk factors associated with suicide ideation/attempt in American Indian/Alaska Native and White youth in Montana. Arch Suicide Res. 2015;19:89-102. doi: 10.1080/13811118.2013.840254.

413. Whitesell NR, Mitchell CM, Kaufman CE, Spicer P, Voices of Indian Teens Project Team. Developmental trajectories of personal and collective self-concept among American Indian adolescents. Child Dev. 2006;77(5):1487-503.

414. Byrnes JP, Miller DC, Schafer WD. Gender differences in risk taking: A meta-analysis. Psychological Bulletin. 1999;125(3):367-83. doi: 10.1037/0033-2909.125.3.367.

415. Polzin PE, O'Donnell D. Economic status of American Indians in Montana. Montana Business Quarterly. 2004;Winter.

416. Foley RM, Pang LS. Alternative Education Programs: Program and Student Characteristics. The High School Journal. 2006;89(3):10-21.

417. Iritani BJ, Hallfors DD, Bauer DJ. Crystal methamphetamine use among young adults in the USA. Addiction. 2007;102:1102-13. doi: 10.1111/j.1360-0443.2007.01847.x.

418. Andersen SR, Belcourt GM, Langwell KM. Building healthy Tribal Nations in Montana and Wyoming through collaborative research and development. Am J Public Health. 2005;95(5):784-9. doi: 10.2105/AJPH.2004.051032.

419. Bubar R, Winokur M, Bartlemay W. Perceptions of methamphetamine use in three western tribal communities: Implications for child abuse in Indian country. Tribal Law and Policy Institute. 2007. 
420. Freese TE, Obert J, Dickow A, Cohen J, Lord RH. Methamphetamine abuse: Issues for special populations. J Psychoactive Drugs. 2000;32(2):177-82.

421. Kronk EA. The emerging problem of methamphetamine: A threat signaling the need to reform criminal jurisdiction in indian country. North Dakota Law Review. 2006;82(4):1249-72.

422. The National Congress Of American Indians. Methamphetamine in Indian country: An American Problem Uniquely Affecting Indian Country. 2006.

423. Steele PD. Child sexual abuse among socially marginalized groups: Cultural and governmental influences perpetuating maltreatment in American Indian country. Forum on Public Policy. 2009(1).

424. Pierce AS. American Indian adolescent girls: Vulnerability to sex trafficking, intervention strategies. Am Indian Alsk Native Ment Health Res. 2012;19(1):37-56. doi:

10.5820/aian.1901.2012.37.

425. Maze of Injustice: The Failure to Protect Indigenous Women from Sexual Violence. New York, NY: Amnesty International Publications, 2007.

426. McManama O'Brien KH, Becker SJ, Spirito A, Simon V, Prinstein MJ. Differentiating adolescent suicide attempters from ideators: Examining the interaction between depression severity and alcohol use. Suicide Life Threat Behav. 2014;44:23-33. doi: 10.1111/sltb.12050.

427. Stack S. Differentiating suicide ideators from attempters: Violence-a research note. Suicide Life Threat Behav. 2014;44(1):46-57. doi: 10.1111/sltb.12054.

428. Taliaferro LA, Muehlenkamp JJ. Risk and protective factors that distinguish adolescents who attempt suicide from those who only consider suicide in the past year. Suicide Life Threat Behav. 2014;44: 6-22. doi: 10.1111/sltb.12046.

429. Klonsky ED, May AM. Differentiating suicide attempters from suicide ideators: A critical frontier for suicidology research. Suicide Life Threat Behav. 2014;44:1-5. doi: 10.1111/sltb.12068.

430. Gone JP. Mental health services for Native Americans in the 21st century United States. Professional Psychology. 2004;35(1):10-8.

431. Levit KR, Kassed CA, Coffey RM, Mark TL, Stranges EM, Buck JA, Vandivort-Warren R. Future funding for mental health and substance abuse: Increasing burdens for the public sector. Health Affairs. 2007;27(6):w513-w22.

432. Corso PS, Mercy JA, Simon TR, Finkelstein EA, Miller TR. Medical costs and productivity losses due to interpersonal and self-directed violence in the United States. Am J Prev Med. 2007;32(6):474-82. doi: 10.1016/j.amepre.2007.02.010.

433. Wexler LM, Gone JP. Culturally responsive suicide prevention in Indigenous communities: Unexamined assumptions and new possibilities. Am J Public Health. 2012;102(5):800-6. doi: 10.2105/Ajph.2011.300432. 
434. Libby AM, Orton HD, Novins DK, Beals J, Manson SM. Childhood physical and sexual abuse and subsequent depressive and anxiety disorders for two American Indian tribes. Psychol Med. 2005;35(3):329-40.

435. Walls ML, Chapple CL, Johnson KD. Strain, emotion, and suicide among American Indian youth. Deviant Behavior. 2007;28:219-46.

436. Wang M-C, Nyutu PN, Tran KK. Coping, reasons for living, and suicide in black college students. Journal of Counseling \& Development. 2012;90:459-66. doi: 10.1002/j.15566676.2012.00057.x.

437. Chandler MJ, Lalonde CE, Sokol BW, Hallett D. Personal persistence, identity development, and suicide: A study of Native and Non-native North American adolescents. Monogr Soc Res Child Dev. 2003;68(2):vii-viii, 1-130.

438. Reynolds WM, Mazza JJ. Assessment of suicidal ideation in inner-city children and young adolescents: Reliability and validity of the Suicidal Ideation Questionnaire-JR. School Psychology Review. 1999;28(1).

439. Innamorati M, Pompili M, Serafini G, Lester D, Erbuto D, Amore M, Tatarelli R, Girardi P. Psychometric properties of the Suicidal History Self-Rating Screening Scale. Arch Suicide Res. 2011;15(1):87-92. doi: 10.1080/13811118.2011.540471.

440. Parker G, Roussos J, Hadzi-Pavlovic D, Mitchell P, Wilhelm K, Austin MP. The development of a refined measure of dysfunctional parenting and assessment of its relevance in patients with affective disorders. Psychological Medicine. 1997;27:1193-203.

441. Williams DR, Yu Y, Jackson JS, Anderson NB. Racial differences in physical and mental health: Socio-economic status, stress and discrimination. Journal of Health Psychology. 1997;2(3):335-51.

442. Cohen S, Williamson GM. Perceived stress in a probability sample of the United States. In: Spacapan S, Oskamp S, editors. The Social Psychology of Health. Newbury Park, CA: Sage; 1988.

443. Radloff LS. The use of the Center for Epidemiologic Studies Depression Scale in adolescents and young adults. J Youth Adolesc. 1991;20(2):149-66.

444. Radloff LS. The CES-D Scale: A self-report depression scale for research in the general population. Applied Psychological Measurement 1977;1:385-401.

445. Beals J, Manson SM, Keane EM, Dick RW. Factorial structure of the Center for Epidemiologic Studies-Depression Scale among American Indian college students.

Psychological Assessment. 1991;3(4):623-7.

446. Armenta BE, Sittner Hartshorn KJ, Whitbeck LB, Crawford DM, Hoyt DR. A longitudinal examination of the measurement properties and predictive utility of the Center for Epidemiologic Studies Depression Scale among North American Indigenous adolescents. Psychological Assessment. 2014;26(4):1347. doi: http://dx.doi.org/10.1037/a0037608. 
447. Spitzer RL, Kroenke K, Williams JBW, Lowe B. A brief measure for assessing generalized anxiety disorder: The GAD-7. Arch Intern Med. 2006;166:1092-7.

448. Hansen M, Andersen TE, Armour C, Elklit A, Palie S, Mackrill T. PTSD-8: A short PTSD inventory. Clinical Practice and Epidemiology in Mental Health. 2010;6:101-8.

449. Sarason IG, Sarason BR, Shearin EN, Pierce GR. A brief measure of social support: Practical and theoretical implications. Journal of Social and Personal Relationships. 1987;4(4):497-510.

450. Whitesell NR, Mitchell CM, Spicer P, Voices of Indian Teens Project Team. A longitudinal study of self-esteem, cultural identity, and academic success among American Indian adolescents. Cultur Divers Ethnic Minor Psychol. 2009;14(1):38-50.

451. Wagnild GM, Young HM. Development and psychometric evaluation of the Resilience Scale. Journal of Nursing Measurement. 1993;1(2):165-78.

452. Wagnild GM. A review of the Resilience Scale. Journal of Nursing Measurement. 2009;17(2):105-13.

453. McCullough ME, Emmons RA, Tsang J. The grateful disposition: A conceptual and empirical topography. J Pers Soc Psychol. 2002;82:112-27.

454. Oetting ER, Beauvais F. Orthogonal cultural identification theory: The cultural identification of minority adolescents. Int J Addict. 1990;25(5A-6A):655-85.

455. Carver CS. You want to measure coping but your protocol's too long: Consider the Brief COPE. International Journal of Behavioral Medicine. 1997;4(1):92-100.

456. Krägeloh CU. A systematic review of studies using the Brief COPE: Religious coping in factor analyses. Religions. 2011;2(3):216-46.

457. Scotti JR, Stevens SB, Jacoby VM, Bracken MR, Freed R, Schmidt E. Trauma in persons with intellectual and developmental disabilities: Reactions of parents and caregivers to research participation. Intellect Dev Disabil. 2012;50(3):199-206.

458. Allison PD. Logistic regression using the SAS system: Theory and application. Cary, NC: SAS Institute and Willey; 2001.

459. Manzo K, Hobbs G, Tiesman H, Stewart J, Gachupin F, Knox SS. Reservation-urban comparison of suicidal ideation and attempts in American Indian youth. Manuscript in Preparation.

460. Adverse childhood experiences reported by adults --- Five states, 2009. MMWR. 2010;59(49):1609-13.

461. Anda RF, Felitti VJ, Bremner JD, Walker JD, Whitfield C, Perry BD, Dube SR, Giles WH. The enduring effects of abuse and related adverse experiences in childhood. A convergence of evidence from neurobiology and epidemiology. Eur Arch Psychiatry Clin Neurosci.

2006;256(3):174-86. 
462. Dube SR, Anda RF, Felitti VJ, Edwards VJ, Williamson DF. Exposure to abuse, neglect, and household dysfunction among adults who witnessed intimate partner violence as children: Implications for health and social service. Violence Vict. 2002;17(1):3-17.

463. Dong M, Anda RF, Dube SR, Giles WH, Felitti VJ. The relationship of exposure to childhood sexual abuse to other forms of abuse, neglect, and household dysfunction during childhood. Child Abuse Negl. 2003;27(6):625-39. doi: 10.1016/S0145-2134(03)00105-4.

464. Dong M, Anda RF, Felitti VJ, Dube SR, Williamson DF, Thompson TJ, Loo CM, Giles $\mathrm{WH}$. The interrelatedness of multiple forms of childhood abuse, neglect, and household dysfunction. Child Abuse Negl. 2004;28(7):771-84. doi: 10.1016/j.chiabu.2004.01.008.

465. Mersky JP, Topitzes J, Reynolds AJ. Impacts of adverse childhood experiences on health, mental health, and substance use in early adulthood: A cohort study of an urban, minority sample in the U.S. Child Abuse Negl. 2013;37(11):917-25. doi: 10.1016/j.chiabu.2013.07.011.

466. Roh S, Burnette CE, Lee KH, Lee YS, Easton SD, Lawler MJ. Risk and protective factors for depressive symptoms among American Indian older adults: Adverse childhood experiences and social support. Aging Ment Health. 2014:1-10.

467. McQuaid RJ, Bombay A, McInnis OA, Matheson K, Anisman H. Childhood adversity, perceived discrimination, and coping strategies in relation to depressive symptoms among First Nations adults in Canada: The moderating role of unsupportive social interactions from ingroup and outgroup members. Cultur Divers Ethnic Minor Psychol. 2014. doi: http://dx.doi.org/10.1037/a0037541.

468. Burnette CE. Indigenous women's resilience and resistance to historical oppression: A case example from the United States. Affilia. 2014. doi: 10.1177/0886109914555215.

469. Yellow Horse Brave Heart M. The return to the sacred path: Healing the historical trauma and historical unresolved grief response among the Lakota through a psychoeducational group intervention. Smith College Studies in Social Work. 1998;68(3):287-305.

470. Seery MD, Holman EA, Silver RC. Whatever does not kill us: Cumulative lifetime adversity, vulnerability, and resilience. J Pers Soc Psychol. 2010;99(6):1025-41.

471. Tsang J-A, Schulwitz A, Carlisle RD. An experimental test of the relationship between religion and gratitude. Psychology of Religion and Spirituality. 2012;4(1):40-55. doi: http://dx.doi.org/10.1037/a0025632.

472. Krause N. Gratitude toward God, stress, and health in late life. Research on Aging. 2006;28(2):163-83.

473. Dillman DA, Smyth JD, Christian LM. When more than one survey mode is needed. Internet, Mail, and Mixed-Mode Surveys: The Tailored Design Method. 3rd ed. Hoboken, N J: John Wiley \& Sons, Inc; 2009.

474. Tucker RP, Judah MR, O'Keefe VM, Mills AC, Lechner WV, Davidson CL, Grant DM, Wingate LR. Humor styles impact the relationship between symptoms of social anxiety and 
depression. Personality and Individual Differences. 2013;55(7):823-7. doi:

10.1016/j.paid.2013.07.008.

475. CDC. Suicide trends among persons aged 10-24 years - United States, 1994-2012. MMWR. 2015;64(08):201-5.

476. Sánchez-Teruel D, \& Robles-Bello, M. A. Protective factors promoting resilience to suicide in young people and adolescents. Papeles del Psicologo. 2014;35:181-92.

477. Fraser SL, Geoffroy D, Chachamovich E, Kirmayer LJ. Changing rates of suicide ideation and attempts among Inuit youth: A gender-based analysis of risk and protective factors. Suicide Life Threat Behav. 2014. doi: 10.1111/sltb.12122.

478. Kading M, Hautala DS, Palombi LC, Aronson BD, Smith RC, Walls ML. Flourishing: American Indian positive mental health. Society and Mental Health. Epub February 2, 2015. doi: $10.1177 / 2156869315570480$. 
APPENDIX A. 


\section{Appendix A.}

Table 1. Prevalence of Suicidality and Select Risk Factors by Gender and Race/Ethnicity for American Indian and White Montana Youth: YRBS, $1999-2011$

\begin{tabular}{|c|c|c|c|c|c|c|c|c|c|}
\hline & & Girls & & & Boys & & Racial/Ethnic [ & Differences & \\
\hline & $\begin{array}{l}\text { American } \\
\text { Indian } \\
n=628 \\
\text { (n) } \%\end{array}$ & $\begin{array}{l}\text { White } \\
n=8814 \\
\text { (n) } \%\end{array}$ & $\begin{array}{c}p \text { value } \\
*<.0005\end{array}$ & $\begin{array}{l}\text { American } \\
\text { Indian } \\
n=695 \\
(\mathrm{n}) \%\end{array}$ & $\begin{array}{l}\text { White } \\
n=8715 \\
\text { (n) } \%\end{array}$ & $\begin{array}{r}p \text { value } \\
*<.0005\end{array}$ & $\begin{array}{l}\text { American } \\
\text { Indian } \\
n=1323 \\
\text { (n) } \%\end{array}$ & $\begin{array}{l}\text { White } \\
n=17529 \\
\text { (n) } \%\end{array}$ & $\begin{array}{r}\mathrm{p} \text { value } \\
*<.0005\end{array}$ \\
\hline \multicolumn{10}{|l|}{ Suicidal Thoughts/Behaviors } \\
\hline Consider & (165) $26.8 \%{ }^{*}$ & (1813) $20.7 \%$ & .0003 & (117) $17.1 \% *$ & (1019) $11.9 \%$ & $<.0001$ & (282) $21.7 \%^{*}$ & (2832) $16.4 \%$ & $<.0001$ \\
\hline Plan & (131) $21.0 \%$ & (1412) $16.1 \%$ & .0014 & (90) $13.1 \%$ & (931) $10.8 \%$ & .0656 & (221) $16.9 \%$ & (2343) $13.5 \%$ & .0006 \\
\hline Attempt & (106) $19.6 \%$ * & (731) $9.1 \%$ & $<.0001$ & (63) $11.5 \% *$ & (380) $5.0 \%$ & $<.0001$ & (169) $15.5 \% *$ & (1111) $7.1 \%$ & $<.0001$ \\
\hline Injury with attempt & (35) $6.5 \% *$ & (215) $2.7 \%$ & $<.0001$ & (19) $3.4 \%$ & (146) $1.9 \%$ & .0148 & (54) $4.9 \% *$ & (361) $2.3 \%$ & $<.0001$ \\
\hline Sadness/Hopelessness & (267) $43.2 \%$ * & (2748) $31.4 \%$ & $<.0001$ & (169) $24.7 \%{ }^{*}$ & (1559) $18.1 \%$ & $<.0001$ & (436) $33.5 \%$ * & (4307) $24.8 \%$ & $<.0001$ \\
\hline \multicolumn{10}{|l|}{ Alcohol, Tobacco, Marijuana } \\
\hline Current drinking & (305) $53.8 \% *$ & (3779) $45.1 \%$ & $<.0001$ & (310) $50.8 \%$ & (3929) 48.0\% & .1773 & (615) $52.3 \%$ * & (7708) $46.6 \%$ & .0002 \\
\hline Binge drinking & (245) $41.0 \%$ * & (2651) $30.6 \%$ & $<.0001$ & (255) $38.8 \%$ & (3010) $35.4 \%$ & .0810 & (500) $39.9 \% *$ & (5661) $33.0 \%$ & $<.0001$ \\
\hline Riding w/someone drinking & (272) $43.8 \%$ * & (2879) $32.9 \%$ & $<.0001$ & (291) 42.4\%* & (2786) $32.3 \%$ & $<.0001$ & (563) $43.0 \%$ * & (5665) $32.6 \%$ & $<.0001$ \\
\hline Driving while drinking & (117) $19.2 \%$ & (1228) $14.1 \%$ & .0006 & (120) $18.2 \%$ & (1600) $18.8 \%$ & .7088 & (237) $18.7 \%$ & (2828) $16.4 \%$ & .0372 \\
\hline Current smoking & (300) $52.3 \% *$ & (1703) $20.1 \%$ & $<.0001$ & (240) $39.4 \% *$ & (1634) $19.7 \%$ & $<.0001$ & (540) $45.7 \% *$ & (3337) $19.9 \%$ & $<.0001$ \\
\hline Current marijuana use & (265) $43.9 \% *$ & (1576) $18.1 \%$ & $<.0001$ & (275) $42.1 \% *$ & (1859) $21.9 \%$ & $<.0001$ & (540) $43.0 \%$ * & (3435) $20.0 \%$ & $<.0001$ \\
\hline \multicolumn{10}{|l|}{ Inhalant Drugs } \\
\hline Cocaine & (103) $17.0 \%{ }^{*}$ & (517) $6.0 \%$ & $<.0001$ & (102) $15.2 \%^{*}$ & (650) $7.6 \%$ & $<.0001$ & (205) $16.1 \%{ }^{*}$ & (1167) $6.8 \%$ & $<.0001$ \\
\hline Current cocaine & (43) $7.0 \% *$ & (171) $2.0 \%$ & $<.0001$ & (43) $6.4 \% *$ & (263) $3.1 \%$ & $<.0001$ & (86) $6.7 \% *$ & $(434)$ & $<.0001$ \\
\hline Meth & (116) $18.8 \%$ * & (502) $5.7 \%$ & $<.0001$ & (92) $13.7 \% *$ & (561) $6.5 \%$ & $<.0001$ & (208) $16.1 \% *$ & (1063) $6.1 \%$ & $<.0001$ \\
\hline \multicolumn{10}{|l|}{ Injection Drugs } \\
\hline Heroin & (28) $4.5 \%{ }^{*}$ & (136) $1.6 \%$ & $<.0001$ & (44) $6.5 \% *$ & (252) $2.9 \%$ & $<.0001$ & (72) $5.5 \% *$ & (388) $2.2 \%$ & $<.0001$ \\
\hline Steroids & (43) $6.9 \% *$ & (203) $2.3 \%$ & $<.0001$ & (43) $6.3 \%$ & (339) $3.9 \%$ & .0023 & (86) $6.6 \%{ }^{*}$ & (542) $3.1 \%$ & $<.0001$ \\
\hline Injection drugs & (31) $5.0 \% *$ & (111) $1.3 \%$ & $<.0001$ & (31) $4.6 \%$ & (230) $2.7 \%$ & .0037 & (62) $4.8 \% *$ & (341) $2.0 \%$ & $<.0001$ \\
\hline \multicolumn{10}{|l|}{ Early Risk-Taking Behavior } \\
\hline Smoking under age 13 & (224) $37.8 \% *$ & (1127) $13.4 \%$ & $<.0001$ & (244) $38.1 \% *$ & (1433) $17.4 \%$ & $<.0001$ & (468) $38.0 \%{ }^{*}$ & (2560) $15.4 \%$ & $<.0001$ \\
\hline Drinking under age 13 & (178) $29.6 \%$ * & (1835) $22.0 \%$ & $<.0001$ & (239) $36.7 \%$ & (2649) $32.2 \%$ & .0191 & (417) $33.3 \% *$ & (4484) $27.0 \%$ & $<.0001$ \\
\hline Marijuana use under age 13 & (177) $29.2 \%{ }^{*}$ & (474) $5.4 \%$ & $<.0001$ & (246) $37.5 \%{ }^{*}$ & (826) $9.7 \%$ & $<.0001$ & (423) $33.5 \%$ * & (1300) $7.5 \%$ & $<.0001$ \\
\hline Sex under age 13 & (36) $6.4 \%{ }^{*}$ & (215) $2.6 \%$ & $<.0001$ & (105) $18.1 \% *$ & (463) $5.8 \%$ & $<.0001$ & (141) $12.3 \% *$ & (678) $4.1 \%$ & $<.0001$ \\
\hline
\end{tabular}




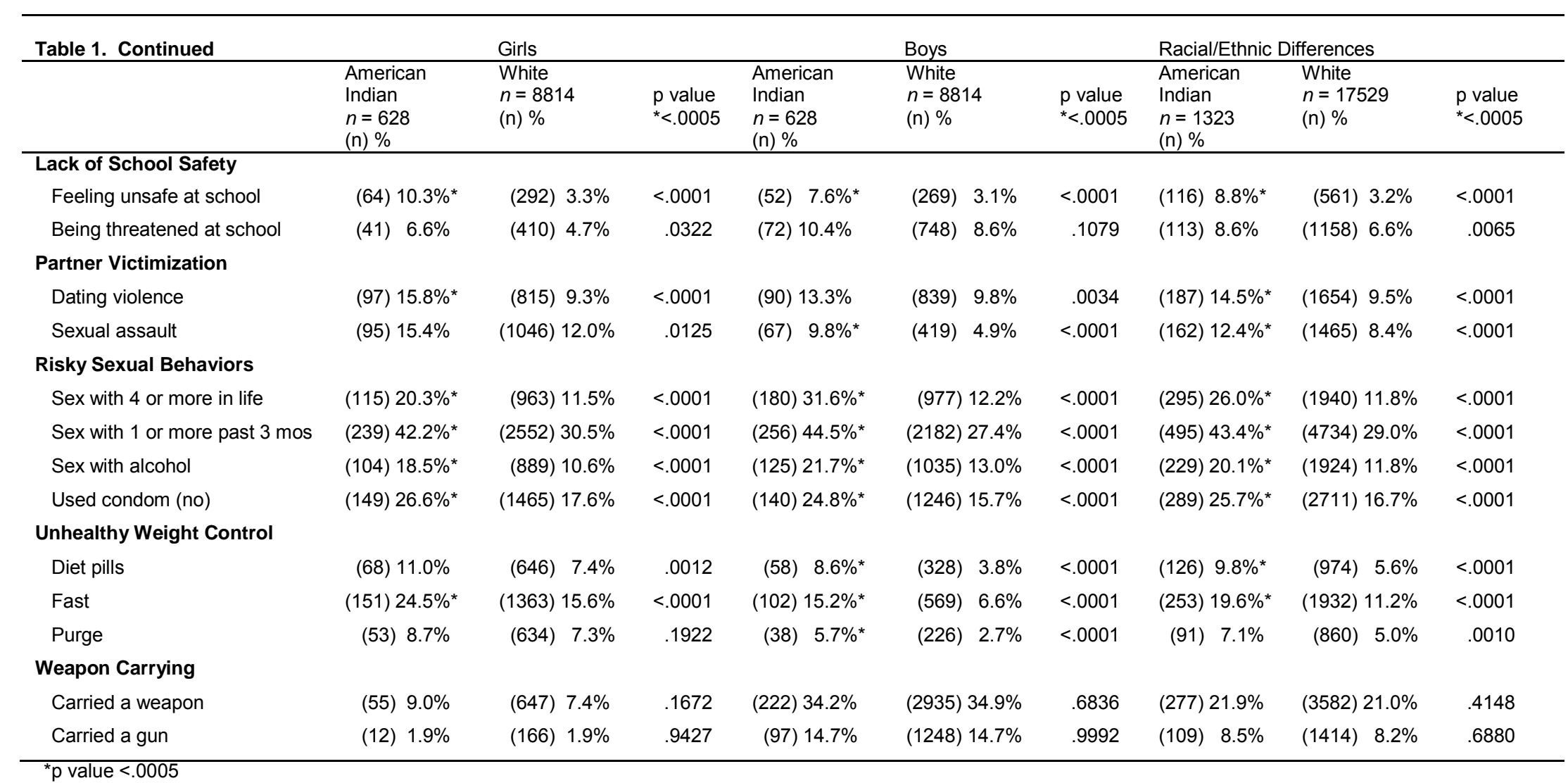


Appendix A.

\begin{tabular}{|c|c|c|c|c|}
\hline & $\begin{array}{l}\text { American Indian Girls } \\
\text { AOR }^{a}(95 \% \mathrm{Cl})\end{array}$ & $\begin{array}{l}\text { American Indian Boys } \\
\text { AOR }^{a}(95 \% \mathrm{Cl})\end{array}$ & $\begin{array}{l}\text { White Girls } \\
\text { AOR }^{a}(95 \% \mathrm{Cl})\end{array}$ & $\begin{array}{l}\text { White Boys } \\
\text { AOR }^{\text {a }}(95 \% \mathrm{Cl})\end{array}$ \\
\hline Sadness/Hopelessness & $4.13(2.51,6.81)^{\star \star \star \star}$ & $6.23(3.59,10.80)^{\star \star \star \star}$ & $6.57(5.76,7.49)^{\star \star \star *}$ & $8.80(7.53,10.28)^{\star \star \star \star}$ \\
\hline Alcohol/Tobacco/Marijuana & $1.13(0.85,1.51)$ & $1.43(1.05,1.94)^{*}$ & $1.13(1.04,1.23)^{\star *}$ & $1.07(0.98,1.17)$ \\
\hline Inhalant Drugs & $0.96(0.63,1.45)$ & $0.79(0.47,1.33)$ & $1.04(0.86,1.26)$ & $1.10(0.92,1.32)$ \\
\hline Injection Drugs & $1.01(0.40,2.59)$ & $1.86(0.79,4.40)$ & $1.64(0.96,2.79)$ & $1.08(0.86,1.36)$ \\
\hline Early Risk-Taking Behaviors & $1.13(0.47,2.73)$ & $1.25(0.51,3.04)$ & $1.55(1.20,2.00)^{\star \star *}$ & $1.83(1.39,2.40)^{\star * * *}$ \\
\hline Lack of School Safety & $1.63(0.74,3.58)$ & $1.15(0.80,1.66)$ & $1.43(1.131 .81)^{\star *}$ & $1.28(1.13,1.45)^{\star \star \star}$ \\
\hline Partner Victimization & $2.01(0.92,4.38)$ & $1.02(0.40,2.62)$ & $1.88(1.47,2.39)^{\star \star \star \star}$ & $1.76(1.27,2.43)^{\star \star \star}$ \\
\hline Risky Sexual Behaviors & $1.14(0.79,1.66)$ & $1.01(0.71,1.45)$ & $1.12(0.99,1.27)$ & $1.29(1.14,1.47)^{\star \star \star *}$ \\
\hline Unhealthy Weight Control & $4.54(1.55,13.33)^{\star \star}$ & $2.21(0.69,7.10)$ & $3.86(2.96,5.03)^{\star \star * \star}$ & $2.15(1.43,3.22)^{\star \star \star}$ \\
\hline Weapon Carrying & $1.59(0.79,3.22)$ & $1.34(1.05,1.72)^{*}$ & $1.16(1.01,1.32)^{*}$ & $1.01(0.95,1.08)$ \\
\hline Age & $0.77(0.62,0.97)^{*}$ & $1.00(0.78,1.28)$ & $0.85(0.81,0.90)^{\star * * *}$ & $0.90(0.84,0.96)^{\star *}$ \\
\hline
\end{tabular}

$\mathrm{AOR}=$ adjusted odds ratio; $\mathrm{Cl}=$ confidence interval

a Bolded odds ratios indicate a statistically significant association comparing those who reported ideation or attempt and those who did not within each group; odds ratios are adjusted for all other model variables.

${ }^{*} p<.05$

$* * p<.01$

$* * * 0<.001$

$* * * * p<.0001$ 
APPENDIX B. 


\section{Appendix B.}

Table 1. Descriptive Data for Suicidal Ideation, Suicide Attempts, and Risk Factor Clusters by Gender for Reservation $(n=3748)$ and Urban ( $n=2669)$ Youth

\begin{tabular}{|c|c|c|c|c|c|c|}
\hline \multirow[t]{2}{*}{ 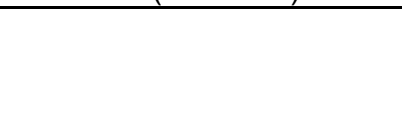 } & \multicolumn{3}{|c|}{ Girls } & \multicolumn{3}{|c|}{ Boys } \\
\hline & $\begin{array}{c}\text { Reservation } \\
n=1860\end{array}$ & $\begin{array}{l}\text { Urban } \\
n=1284\end{array}$ & $\begin{array}{l}p \text { value } \\
*<.004\end{array}$ & $\begin{array}{l}\text { Reservation } \\
n=1888\end{array}$ & $\begin{array}{l}\text { Urban } \\
n=1385\end{array}$ & $\begin{array}{l}\mathrm{p} \text { value } \\
{ }^{*}<.004\end{array}$ \\
\hline Outcome Variable & $(n) \%$ & (n) $\%$ & & (n) $\%$ & (n) $\%$ & \\
\hline Suicidal Ideation ${ }^{a}$ & (276) $15.1 \%$ & (230) $18.2 \%$ & .0194 & (217) $11.9 \%$ & (182) $13.4 \%$ & 1960 \\
\hline Suicide Attempts & (352) 19.2\% & (254) $20.1 \%$ & .5362 & (250) $9.6 \%$ & (219) $10.4 \%$ & .0476 \\
\hline \multicolumn{7}{|l|}{ Risk Factor Clusters ${ }^{b}$} \\
\hline Sadness/Hopelessness & $40.4 \%$ & $49.0 \% *$ & $<.0001$ & $23.1 \%$ & $26.2 \%$ & .0438 \\
\hline Alcohol/Tobacco/Marijuana & $41.9 \%$ * & $35.9 \%$ & $<.0001$ & $39.1 \% *$ & $35.5 \%$ & .0030 \\
\hline Inhalant Drugs & $10.3 \%$ & $11.7 \%$ & .1188 & $9.5 \%$ & $11.3 \%$ & .0447 \\
\hline Injection Drugs & $3.2 \%$ & $5.4 \% *$ & $<.0001$ & $3.9 \%$ & $6.0 \% *$ & .0004 \\
\hline Disordered Eating & $12.2 \%$ & $14.3 \%$ & .0169 & $8.0 \%$ & $8.4 \%$ & .6341 \\
\hline Early Risk-Taking Behavior & $26.5 \%$ & $25.4 \%$ & .3019 & $29.7 \%$ & $30.3 \%$ & .6015 \\
\hline Lack of School Safety & $6.9 \%$ & $7.0 \%$ & .8925 & $7.6 \%$ & $9.3 \%$ & .0256 \\
\hline Partner Victimization & $13.4 \%$ & $18.5 \%$ * & $<.0001$ & $11.7 \%$ & $12.5 \%$ & .3644 \\
\hline Risky Sexual Behaviors & $28.8 \%$ & $27.2 \%$ & .1488 & $31.1 \% *$ & $26.1 \%$ & $<.0001$ \\
\hline Weapon Carrying & $6.1 \%$ & $9.2 \% *$ & $<.0001$ & $22.1 \%$ & $30.0 \%$ * & $<.0001$ \\
\hline
\end{tabular}

* $p$ value $<.004$ (Bonferroni corrected)

a Responded yes to ideation, but not to attempt

${ }^{b}$ Proportion of affirmative responses in each cluster (presented as a percent) 
Appendix B.

\begin{tabular}{|c|c|c|}
\hline & $\begin{array}{l}\text { Reservation Girls } \\
n=1777 \\
\text { AOR }^{\mathrm{b}}(95 \% \mathrm{Cl})\end{array}$ & $\begin{array}{c}\text { Urban Girls } \\
n=1236 \\
\text { AOR }^{\mathrm{b}}(95 \% \mathrm{Cl})\end{array}$ \\
\hline Sadness/Hopelessness & $2.64(1.98,3.50)^{c}$ & $4.41(3.09,6.28)^{c}$ \\
\hline Alcohol/Tobacco/Marijuana & $1.16(1.00,1.35)$ & $1.06(0.89,1.27)$ \\
\hline Inhalant Drugs & $0.73(0.54,0.98)^{f}$ & $0.86(0.63,1.19)$ \\
\hline Injection Drugs & $0.96(0.56,1.65)$ & $0.76(0.44,1.32)$ \\
\hline Disordered Eating & $1.80(1.07,3.02)^{f}$ & $0.65(0.36,1.20)$ \\
\hline Early Risk-Taking Behaviors & $1.01(0.61,1.67)$ & $0.61(0.34,1.11)$ \\
\hline Lack of School Safety & $1.09(0.82,1.45)$ & $1.06(0.79,1.44)$ \\
\hline Partner Victimization & $1.64(1.01,2.66)^{f}$ & $1.92(1.15,3.20)^{f}$ \\
\hline Risky Sexual Behaviors & $0.98(0.87,1.11)$ & $0.99(0.86,1.14)$ \\
\hline Weapon Carrying & $0.90(0.65,1.24)$ & $0.99(0.77,1.27)$ \\
\hline
\end{tabular}

Abbreviations: AOR, adjusted odds ratio

${ }^{\text {a }}$ Responded yes to ideation, but not to attempt.

${ }^{\mathrm{b}}$ Odds ratios are adjusted for age and all other model variables.

c $p<.0001$

d $p<.001$

e $p<.01$

${ }^{f} p<.05$ 
Appendix B.

\begin{tabular}{|c|c|c|}
\hline & $\begin{array}{c}\text { Reservation Boys } \\
n=1750 \\
\text { AOR }^{\mathrm{b}}(95 \% \mathrm{Cl})\end{array}$ & $\begin{array}{c}\text { Urban Boys } \\
n=1331 \\
\text { AOR }^{\mathrm{b}}(95 \% \mathrm{Cl})\end{array}$ \\
\hline Sadness/Hopelessness & $3.83(2.78,5.27)^{c}$ & $5.34(3.77,7.56)^{c}$ \\
\hline Alcohol/Tobacco/Marijuana & $1.00(0.85,1.19)$ & $1.09(0.92,1.30)$ \\
\hline Inhalant Drugs & $0.88(0.65,1.19)$ & $1.06(0.76,1.47)$ \\
\hline Injection Drugs & $0.96(0.61,1.52)$ & $0.73(0.43,1.24)$ \\
\hline Disordered Eating & $1.71(0.87,3.37)$ & $1.80(0.83,3.89)$ \\
\hline Early Risk-Taking Behaviors & $1.74(1.02,2.99)^{f}$ & $1.10(0.62,1.95)$ \\
\hline Lack of School Safety & $0.98(0.80,1.21)$ & $0.94(0.77,1.16)$ \\
\hline Partner Victimization & $2.22(1.30,3.78)^{\mathrm{e}}$ & $1.17(0.64,2.15)$ \\
\hline Risky Sexual Behaviors & $0.97(0.84,1.13)$ & $0.95(0.81,1.12)$ \\
\hline Weapon Carrying & $1.19(1.03,1.37)^{f}$ & $0.97(0.84,1.13)$ \\
\hline
\end{tabular}

Abbreviations: AOR, adjusted odds ratio

a Responded yes to ideation, but not to attempt.

${ }^{b}$ Odds ratios are adjusted for age and all other model variables.

${ }^{c} p<.0001$

d $p<.001$

e $p<.01$

f $p<.05$ 
Appendix B.

\begin{tabular}{|c|c|c|}
\hline & $\begin{array}{c}\text { Reservation Girls } \\
n=1778 \\
\text { AOR }^{\mathrm{a}}(95 \% \mathrm{Cl})\end{array}$ & $\begin{array}{c}\text { Urban Girls } \\
n=1237 \\
\text { AOR }^{\mathrm{a}}(95 \% \mathrm{Cl})\end{array}$ \\
\hline Sadness/Hopelessness & $2.37(1.82,3.07)^{b}$ & $2.25(1.61,3.15)^{b}$ \\
\hline Alcohol/Tobacco/Marijuana & $1.13(0.99,1.30)$ & $1.02(0.86,1.20)$ \\
\hline Inhalant Drugs & $0.88(0.69,1.11)$ & $1.39(1.07,1.82)^{e}$ \\
\hline Injection Drugs & $1.14(0.74,1.76)$ & $1.22(0.76,1.95)$ \\
\hline Unhealthy Weight Control & $1.96(1.20,3.20)^{d}$ & $2.88(1.67,4.96)^{b}$ \\
\hline Early Risk-Taking Behaviors & $2.40(1.53,3.77)^{c}$ & $1.92(1.10,3.35)^{\mathrm{e}}$ \\
\hline Lack of School Safety & $1.33(1.03,1.78)^{\mathrm{e}}$ & $1.53(1.12,2.10)^{d}$ \\
\hline Partner Victimization & $2.09(1.34,3.26)^{c}$ & $1.88(1.15,3.08)^{\mathrm{e}}$ \\
\hline Risky Sexual Behaviors & $1.00(0.89,1.12)$ & $1.05(0.91,1.21)$ \\
\hline Weapon Carrying & $1.32(1.02,1.70)^{\mathrm{e}}$ & $1.27(1.01,1.59)^{\mathrm{e}}$ \\
\hline
\end{tabular}

Abbreviations: AOR, adjusted odds ratio

a Odds ratios are adjusted for age and all other model variables.

${ }^{b} p<.0001$

${ }^{c} p<.001$

d $p<.01$

e $p<.05$ 
Appendix B.

\begin{tabular}{|c|c|c|}
\hline & $\begin{array}{c}\text { Reservation Boys } \\
n=1751 \\
\text { AOR }^{\mathrm{a}}(95 \% \mathrm{Cl})\end{array}$ & $\begin{array}{c}\text { Urban Boys } \\
n=1312 \\
\text { AOR }^{\mathrm{a}}(95 \% \mathrm{Cl})\end{array}$ \\
\hline Sadness/Hopelessness & $2.75(2.02,3.74)^{b}$ & $2.03(1.44,2.85)^{b}$ \\
\hline Alcohol/Tobacco/Marijuana & $1.16(0.99,1.35)$ & $1.16(0.999,1.36)$ \\
\hline Inhalant Drugs & $1.19(0.92,1.54)$ & $0.95(0.71,1.27)$ \\
\hline Injection Drugs & $1.31(0.89,1.91)$ & $1.36(0.89,2.06)$ \\
\hline Unhealthy Weight Control & $0.99(0.49,1.97)$ & $3.05(1.53,5.92)^{d}$ \\
\hline Early Risk-Taking Behaviors & $1.42(0.84,2.37)$ & $1.96(1.14,3.36)^{\mathrm{e}}$ \\
\hline Lack of School Safety & $1.26(1.05,1.51)^{\mathrm{e}}$ & $1.20(1.01,1.42)^{\mathrm{e}}$ \\
\hline Partner Victimization & $0.96(0.55,1.67)$ & $1.81(1.06,3.10)^{\mathrm{e}}$ \\
\hline Risky Sexual Behaviors & $0.97(0.84,1.12)$ & $1.01(0.86,1.18)$ \\
\hline Weapon Carrying & $1.03(0.90,1.18)$ & $0.99(0.86,1.14)$ \\
\hline
\end{tabular}

Abbreviations: AOR, adjusted odds ratio

${ }^{a}$ Odds ratios are adjusted for age and all other model variables.

${ }^{b} p<.0001$

c $p<.001$

d $p<.01$

e $p<.05$ 
APPENDIX C. 
Appendix C.

Figure 1. Culturally Informed Stress-Coping Model for Suicide Risk for Montana-Wyoming Tribal College Students

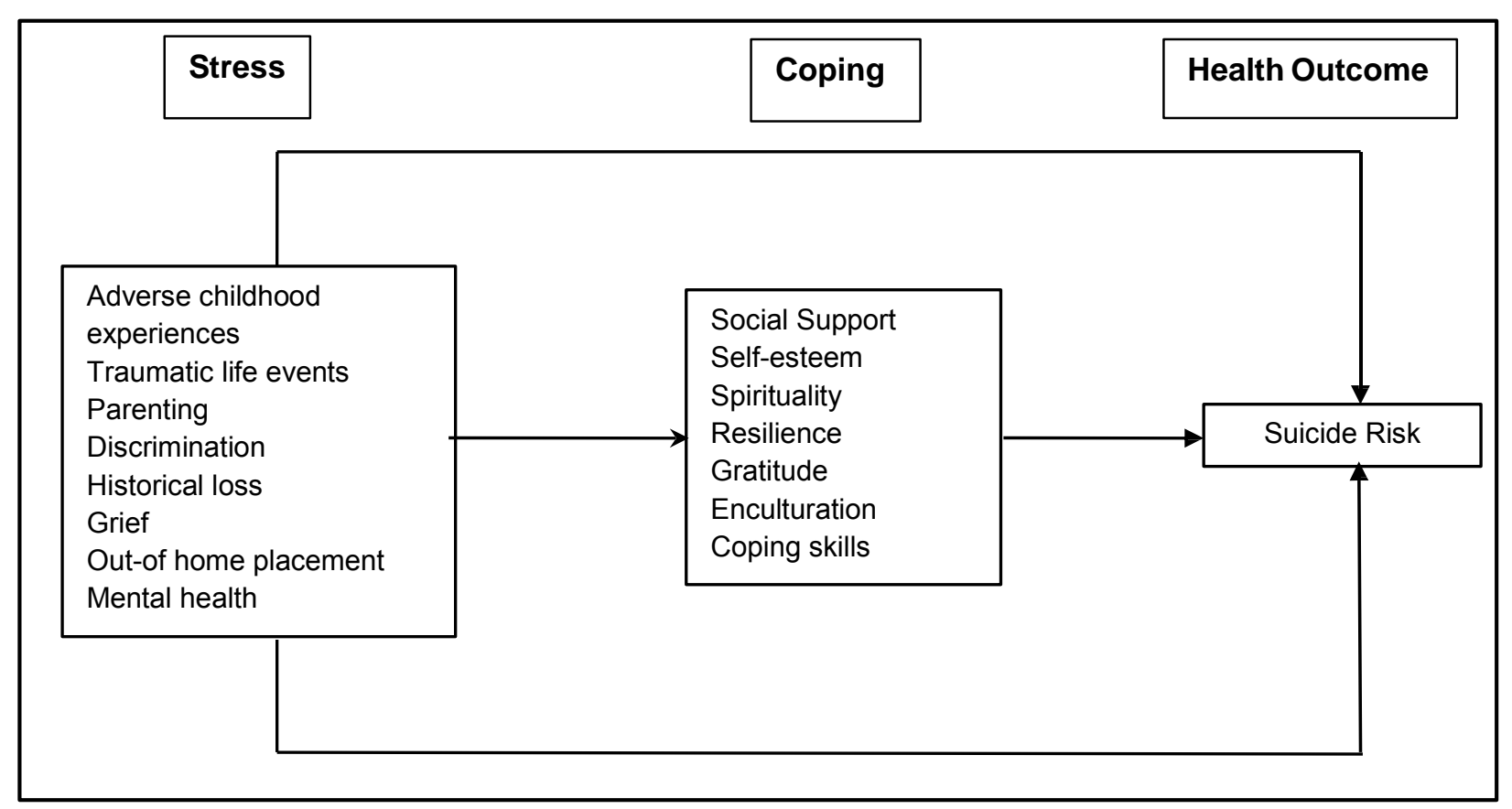




\section{Appendix C.}

Table 1. Lifetime and Past 12 Month Prevalence of Suicidality and Suicide Risk Scores for Montana-Wyoming American Indian Tribal College Students: 2012-2013

\begin{tabular}{|c|c|}
\hline & $\begin{array}{l}\text { Total } \\
\mathrm{n}(\%)\end{array}$ \\
\hline \multicolumn{2}{|l|}{ Suicidality } \\
\hline Lifetime ideation $(n=172)$ & $89(51.7 \%)$ \\
\hline Lifetime plans $(n=185)$ & $60(32.4 \%)$ \\
\hline Lifetime tell someone $(n=177)$ & $46(26.0 \%)$ \\
\hline Lifetime attempts $(n=182)$ & $49(26.9 \%)$ \\
\hline 1 attempt & $27(14.8 \%)$ \\
\hline More than 1 attempt & $22(12.1 \%)$ \\
\hline Past 12-month ideation $(n=175)$ & $47(26.9 \%)$ \\
\hline Past 12-month plans $(n=183)$ & $11(6.0 \%)$ \\
\hline Past 12-month tell someone $(n=184)$ & $17 \quad(9.2 \%)$ \\
\hline Past 12-month attempts* $(n=185)$ & - \\
\hline \multicolumn{2}{|l|}{ Suicide risk score $(n=187)$} \\
\hline 0 & $95(50.8 \%)$ \\
\hline 1 & $5 \quad(2.7 \%)$ \\
\hline 2 & $14(7.5 \%)$ \\
\hline 3 & $10(5.4 \%)$ \\
\hline 4 & $20(10.7 \%)$ \\
\hline 5 & $22(11.8 \%)$ \\
\hline 6 & $13(7.0 \%)$ \\
\hline 7 or higher & $8(4.3 \%)$ \\
\hline Total & $187(100.0 \%)$ \\
\hline
\end{tabular}

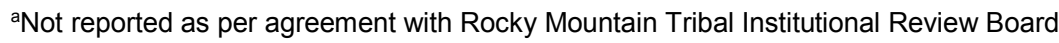


Appendix C.

Table 2. Socio-Demographic Characteristics and Bivariate Models of Suicide Risk for Montana-Wyoming American Indian Tribal College Students: 2012-2013

\begin{tabular}{|c|c|c|}
\hline & $\mathrm{n}(\%)$ & $\begin{array}{c}\mathrm{OR}^{\mathrm{a}}(95 \% \mathrm{Cl}) \\
\text { Bivariate }\end{array}$ \\
\hline Age group $(n=187)$ & & $1.13(0.90,1.40)$ \\
\hline $18-24$ years & $102(54.6 \%)$ & \\
\hline$>=25$ years & $85(45.5 \%)$ & \\
\hline Gender $(n=184)$ & & $0.82(0.65,1.03)$ \\
\hline Men & $65(35.3 \%)$ & \\
\hline Women & $119(64.7 \%)$ & \\
\hline Current household income $(n=178)$ & & $0.87(0.82,0.92)$ \\
\hline$<\$ 10,000 /$ year & $98(55.1 \%)$ & \\
\hline Where spent most of childhood $(n=187)$ & & $1.08(0.79,1.48)$ \\
\hline On or near a reservation & $159(85.0 \%)$ & \\
\hline Mother's education $(n=177)$ & & $1.09(0.87,1.37)$ \\
\hline High school degree or less & $75(40.1 \%)$ & \\
\hline \multicolumn{3}{|l|}{ Boarding school attendance } \\
\hline Participant $(n=186)$ & $26(14.0 \%)$ & $0.67(0.47,0.95)$ \\
\hline \multicolumn{3}{|l|}{ Parents } \\
\hline Mother $(n=185)$ & $50(27.0 \%)$ & $1.15(0.90,1.47)$ \\
\hline Father $(n=182)$ & $33(18.1 \%)$ & $1.34(1.02,1.76)$ \\
\hline Grandparents $(n=183)$ & & $0.98(0.90,1.07)$ \\
\hline \multicolumn{3}{|l|}{ Foster care/adoption } \\
\hline Participant (combined) $(n=187)$ & & $1.35(1.11,1.63)$ \\
\hline Foster care $(n=185)$ & $28(15.1 \%)$ & $1.44(1.08,1.92)$ \\
\hline Adoption $(n=180)$ & $17 \quad(9.4 \%)$ & $1.57(1.11,2.23)$ \\
\hline \multicolumn{3}{|l|}{ Parents $(n=187)$} \\
\hline Mother $(n=186)$ & $22(11.8 \%)$ & $1.62(1.19,2.21)$ \\
\hline Father $(n=185)$ & $18(9.7 \%)$ & $1.14(0.80,1.64)$ \\
\hline Grandparents $(n=182)$ & & $1.16(0.89,1.51)$ \\
\hline Generational out-of home placement ${ }^{\mathrm{b}}(n=187)$ & & $1.06(1.01,1.13)$ \\
\hline
\end{tabular}

$\mathrm{OR}=$ odds ratio; $\mathrm{Cl}=$ confidence interval

${ }^{a}$ Bolded odds ratios indicate a statistically significant association between the independent variable and suicide risk score bIncludes 2 generations of boarding school attendance (parents, grandparents) and 3 generations of foster care/adoption (participant, parents, grandparents) 
Appendix C.

Table 3. Scale Psychometrics and Bivariate Models of Suicide Risk for Montana-Wyoming American Indian Tribal College Students: 2012-2013

\begin{tabular}{|c|c|c|c|c|c|}
\hline & Scale Range & \multicolumn{2}{|c|}{$\begin{array}{l}\text { Mean } \\
(S D)\end{array}$} & Cronbach's $\alpha$ & $\begin{array}{c}\mathrm{OR}^{\mathrm{a}}(95 \% \mathrm{Cl}) \\
\text { Bivariate }\end{array}$ \\
\hline \multicolumn{6}{|l|}{ Dependent Variable } \\
\hline Suicide risk & $0-11$ & 2.12 & $(2.51)$ & .82 & \\
\hline \multicolumn{6}{|l|}{ Hypothesized Stressors } \\
\hline Adverse childhood events (ACE) & $0-16$ & 5.09 & $(3.46)$ & .80 & $9.32(5.71,15.23)$ \\
\hline Traumatic events inventory & $0-24$ & 5.73 & $(4.11)$ & .82 & $11.82(6.53,21.38)$ \\
\hline Parental control (mom) & $4-20$ & 12.77 & $(2.86)$ & .57 & $1.69(1.44,1.98)$ \\
\hline Everyday discrimination & $9-54$ & 23.54 & (10.97) & .91 & $1.44(1.32,1.57)$ \\
\hline Perceived stress & $10-50$ & 28.27 & $(6.51)$ & .82 & $2.19(1.83,2.62)$ \\
\hline Historical loss & $12-72$ & 35.94 & $(14.44)$ & .94 & $1.46(1.33,1.60)$ \\
\hline Grief & $12-60$ & 32.54 & $(11.60)$ & .92 & $1.32(1.17,1.49)$ \\
\hline \multicolumn{6}{|l|}{ Mental health } \\
\hline Depressive symptoms & $20-80$ & 36.48 & $(10.54)$ & .89 & $3.36(2.76,4.10)$ \\
\hline Anxiety symptoms & $7-28$ & 12.01 & $(5.27)$ & .94 & $2.01(1.83,2.42)$ \\
\hline Post-traumatic stress symptoms & $8-32$ & 15.12 & (7.11) & .94 & $1.71(1.51,1.94)$ \\
\hline \multicolumn{6}{|l|}{ Hypothesized Protective Factors } \\
\hline Social support & $0-54$ & 15.65 & $(10.40)$ & .96 & $0.90(0.84,0.96)$ \\
\hline Self-esteem & $6-24$ & 19.23 & $(3.29)$ & .81 & $0.46(0.37,0.56)$ \\
\hline Spirituality & $3-15$ & 10.92 & (3.28) & .86 & $1.19(1.07,1.32)$ \\
\hline Resilience & $14-98$ & 80.43 & $(16.41)$ & .97 & $1.00(0.91,1.11)$ \\
\hline Gratitude & $6-42$ & 34.87 & $(6.61)$ & .76 & $0.70(0.64,0.78)$ \\
\hline American Indian identity & $8-40$ & 24.83 & $(7.53)$ & .89 & $1.04(0.92,1.16)$ \\
\hline \multicolumn{6}{|l|}{ Brief COPE 2-item Subscales $^{b}$} \\
\hline Active & $2-8$ & 5.82 & $(1.66)$ & .61 & $1.17(1.01,1.36)$ \\
\hline Planning & $2-8$ & 5.79 & $(1.72)$ & .77 & $1.03(0.91,1.18)$ \\
\hline Positive reframing & $2-8$ & 5.74 & $(1.72)$ & .80 & $0.97(0.85,1.11)$ \\
\hline Acceptance & $2-8$ & 5.61 & $(1.64)$ & .56 & $1.14(0.99,1.30)$ \\
\hline Humor & $2-8$ & 4.75 & $(1.85)$ & .75 & $1.39(1.23,1.57)$ \\
\hline Religious/spiritual & $2-8$ & 5.38 & (2.12) & .85 & $1.17(1.05,1.30)$ \\
\hline Emotional support & $2-8$ & 5.20 & (1.88) & .80 & $0.88(0.78,0.99)$ \\
\hline Instrumental support & $2-8$ & 5.41 & $(1.80)$ & .78 & $0.88(0.78,1.00)$ \\
\hline Self-distraction & $2-8$ & 5.61 & (1.52) & .27 & $1.67(1.43,1.95)$ \\
\hline Denial & $2-8$ & 3.23 & $(1.38)$ & .65 & $1.53(1.33,1.77)$ \\
\hline Venting & $2-8$ & 4.32 & (1.62) & .54 & $1.63(1.42,1.87)$ \\
\hline Substance use & $2-8$ & 3.39 & $(1.87)$ & .90 & $1.46(1.31,1.63)$ \\
\hline Behavioral disengagement & $2-8$ & 3.39 & $(1.46)$ & .58 & $1.77(1.54,2.03)$ \\
\hline Self-blame & $2-8$ & 4.37 & $(1.72)$ & .64 & $1.91(1.68,2.17)$ \\
\hline \multicolumn{6}{|l|}{ Coping Factors } \\
\hline Constructive coping & $16-64$ & 44.59 & (9.59) & .88 & $1.17(0.97,1.40)$ \\
\hline Non-constructive coping & $12-48$ & 23.43 & $(6.18)$ & .80 & $3.22(2.61,3.99)$ \\
\hline
\end{tabular}

$\mathrm{OR}=$ odds ratio $\mathrm{Cl}=$ confidence interval

aBolded odds ratios indicate a statistically significant association between the independent variable and suicide risk score

${ }^{\text {b}}$ Two-item scales are less reliable than multi-item scales; assess Cronbach's $\alpha$ with caution 


\section{Appendix C.}

Table 4. Summary of Principal Component Factor Loadings for Brief COPE Subscales for Montana-Wyoming American Indian Tribal College Students: 2012-2013

\begin{tabular}{lcc}
\hline Brief COPE Subscales & Rotated (Varimax) & Factor Loadings \\
\hline & $\begin{array}{c}\text { Factor } 1 \\
\text { Constructive } \\
\text { Coping }\end{array}$ & $\begin{array}{c}\text { Foctor } 2 \\
\text { Coping }\end{array}$ \\
\hline Self-distraction & $.50^{*}$ & .26 \\
Active & $.77^{*}$ & -.04 \\
Denial & -.03 & $.55^{*}$ \\
Substance use & -.06 & $.55^{*}$ \\
Emotional support & $.67^{*}$ & -.04 \\
Instrumental support & $.73^{*}$ & -.11 \\
Behavioral disengagement & -.14 & $.74^{*}$ \\
Venting & .31 & $.71^{*}$ \\
Positive reframing & $.74^{*}$ & .09 \\
Planning & $.82^{*}$ & .09 \\
Humor & $.38^{*}$ & $.48^{*}$ \\
Acceptance & $.72^{*}$ & .15 \\
Religious/Spiritual & $.51^{*}$ & -.01 \\
Self-blame & .08 & $.73^{*}$ \\
\hline
\end{tabular}

*Values greater than .35 
Appendix C.

Table 5. Multiple Stressor Models of Suicide Risk for Montana-Wyoming American Indian Tribal College Students: 2012-2013

\begin{tabular}{|c|c|c|c|c|}
\hline & $\mathrm{AOR}^{\mathrm{a}}(95 \% \mathrm{Cl})$ & $\mathrm{AOR}^{\mathrm{a}}(95 \% \mathrm{Cl})$ & $\mathrm{AOR}^{\mathrm{a}}(95 \% \mathrm{Cl})$ & $\mathrm{AOR}^{\mathrm{a}}(95 \% \mathrm{Cl})$ \\
\hline STRESSORS & $\begin{array}{c}\text { Model } 1 \\
n=147 \mathrm{AIC}=1470\end{array}$ & $\begin{array}{c}\text { Model } 2 \\
n=171 \text { AIC }=1654\end{array}$ & $\begin{array}{c}\text { Model } 3 \\
n=168 \text { AIC }=1624\end{array}$ & $\begin{array}{c}\text { Model } 4 \\
n=168 \mathrm{AIC}=1624\end{array}$ \\
\hline $\mathrm{ACE}^{\mathrm{b}}$ & $4.50(2.36,8.57)$ & $4.33(2.37,7.93)$ & $3.71(2.00,6.89)$ & $3.91(2.11,7.22)$ \\
\hline Traumatic events inventory & $2.52(1.07,5.98)$ & $2.94(1.35,6.41)$ & $2.34(1.06,5.19)$ & $2.23(1.01,4.93)$ \\
\hline Parental control (mom) & $1.37(1.13,1.66)$ & $1.36(1.15,1.62)$ & $1.25(1.04,1.49)$ & $1.25(1.04,1.49)$ \\
\hline Discrimination & $1.25(1.12,1.40)$ & $1.22(1.09,1.36)$ & $1.16(1.04,1.30)$ & $1.17(1.05,1.31)$ \\
\hline Perceived stress & $1.37(1.09,1.73)$ & $1.35(1.10,1.66)$ & $1.15(0.92,1.45)$ & \\
\hline Historical loss & $1.30(1.14,1.48)$ & $1.24(1.11,1.39)$ & $1.22(1.09,1.37)$ & $1.22(1.09,1.37)$ \\
\hline Grief & $0.92(0.78,1.07)$ & & & \\
\hline Generational out-of-home placement ${ }^{c}$ & $0.88(0.81,0.96)$ & $0.89(0.82,0.96)$ & $0.92(0.85,0.99)$ & $0.92(0.85,1.00)$ \\
\hline Depressive symptoms & & & $1.58(1.19,2.10)$ & $1.70(1.31,2.20)$ \\
\hline
\end{tabular}

$\mathrm{AOR}=$ adjusted odds ratio; $\mathrm{Cl}=$ confidence interval

aBolded odds ratios indicate a statistically significant association between the independent variable and suicide risk; odds ratios are adjusted for all other model variables ACE: Adverse Childhood Experiences

Includes 2 generations of boarding school attendance (parents, grandparents) and 3 generations of foster care/adoption (participant, parents, grandparents) 
Appendix C.

Table 6. Multiple Protective and Brief COPE Factors of Suicide Risk for Montana-Wyoming American Indian Tribal College Students: 2012-2013

\begin{tabular}{|c|c|c|}
\hline & $\mathrm{AOR}^{a}(\mathrm{Cl})$ & $\mathrm{AOR}^{\mathrm{a}}(\mathrm{Cl})$ \\
\hline & $\begin{array}{c}\text { Model } 1 \\
n=165 \text { AIC }=1675\end{array}$ & $\begin{array}{c}\text { Model } 2 \\
n=165 \mathrm{AlC}=1649\end{array}$ \\
\hline Boarding school attendance $^{b}$ & $0.41(0.26,0.63)$ & $0.43(0.28,0.67)$ \\
\hline Social Support & $0.91(0.83,0.98)$ & $0.90(0.83,0.98)$ \\
\hline Self-esteem & $0.47(0.36,0.60)$ & $0.63(0.47,0.83)$ \\
\hline Spirituality & $1.36(1.20,1.54)$ & $1.31(1.15,1.49)$ \\
\hline Resilience & $1.46(1.27,1.69)$ & $1.26(1.07,1.47)$ \\
\hline Gratitude & $0.64(0.56,0.74)$ & $0.69(0.60,0.80)$ \\
\hline American Indian identity & $1.04(0.90,1.19)$ & $1.04(0.90,1.19)$ \\
\hline Constructive coping & & $1.21(0.93,1.58)$ \\
\hline Non-constructive coping & & $1.85(1.42,2.41)$ \\
\hline
\end{tabular}

$\mathrm{AOR}=$ adjusted odds ratio; $\mathrm{Cl}=$ confidence interval

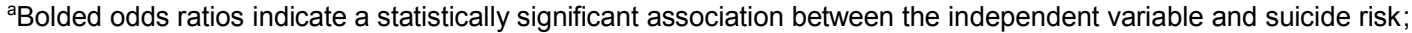
odds ratios are adjusted for all other model variables

${ }^{\mathrm{b}} \mathrm{Participant}$ only 
Appendix C.

Table 7. Multiple Stress-Coping Models of Suicide Risk for Montana-Wyoming American Indian Tribal College Students: 2012-2013

\begin{tabular}{|c|c|c|c|c|}
\hline & $\operatorname{AOR}^{a}(95 \% \mathrm{Cl})$ & $\operatorname{AOR}^{a}(95 \% \mathrm{Cl})$ & $\operatorname{AOR}^{a}(95 \% \mathrm{Cl})$ & $\operatorname{AOR}^{a}(95 \% \mathrm{Cl})$ \\
\hline & $\begin{array}{c}\text { Model } 1 \\
n=160 \mathrm{AIC}=1534\end{array}$ & $\begin{array}{c}\text { Model } 2 \\
n=160 \mathrm{AIC}=1532\end{array}$ & $\begin{array}{c}\text { Model 3 } \\
n=160 \mathrm{AIC}=1518\end{array}$ & $\begin{array}{c}\text { Model } 4 \\
n=160 \mathrm{AIC}=1519\end{array}$ \\
\hline $\mathrm{ACE}^{\mathrm{b}}$ & $3.88(2.02,7.45)$ & $3.49(1.80,6.77)$ & $2.89(1.46,5.69)$ & $2.79(1.41,5.51)$ \\
\hline Traumatic events inventory & $2.49(1.11,5.55)$ & $2.16(0.95,4.89)$ & $2.47(1.09,5.64)$ & $2.23(0.96,5.19)$ \\
\hline Parental control (mom) & $1.33(1.11,1.60)$ & $1.27(1.05,1.54)$ & $1.28(1.06,1.55)$ & $1.25(1.03,1.51)$ \\
\hline Discrimination & $1.10(0.96,1.24)$ & $1.07(0.95,1.21)$ & $1.07(0.94,1.20)$ & $1.05(0.93,1.19)$ \\
\hline Historical loss & $1.15(1.01,1.30)$ & $1.13(1.00,1.28)$ & $1.10(0.98,1.25)$ & $1.10(0.97,1.25)$ \\
\hline Generational out-of-home placement ${ }^{c}$ & $0.93(0.85,1.01)$ & $0.94(0.86,1.02)$ & $0.93(0.85,1.01)$ & $0.93(0.86,1.02)$ \\
\hline Boarding school attendance ${ }^{d}$ & $0.47(0.31,0.73)$ & $0.45(0.29,0.71)$ & $0.46(0.30,0.71)$ & $0.45(0.29,0.69)$ \\
\hline Social support & $0.91(0.84,0.99)$ & $0.91(0.83,0.99)$ & $0.89(0.81,0.97)$ & $0.89(0.81,0.97)$ \\
\hline Self-esteem & $0.73(0.55,0.97)$ & $0.79(0.59,1.07)$ & $0.85(0.63,1.16)$ & $0.89(0.65,1.21)$ \\
\hline Spirituality & $1.35(1.17,1.55)$ & $1.34(1.16,1.54)$ & $1.34(1.16,1.54)$ & $1.33(1.16,1.54)$ \\
\hline Resilience & $1.26(1.06,1.48)$ & $1.24(1.05,1.47)$ & $1.18(0.99,1.41)$ & $1.18(0.99,1.40)$ \\
\hline Gratitude & $0.72(0.62,0.83)$ & $0.75(0.64,0.87)$ & $0.73(0.63,0.85)$ & $0.75(0.64,0.87)$ \\
\hline American Indian identity & $1.03(0.87,1.20)$ & $1.04(0.88,1.22)$ & $1.05(0.89,1.24)$ & $1.06(0.90,1.24)$ \\
\hline Depressive symptoms & & $1.35(1.00,1.82)$ & & $1.20(0.88,1.64)$ \\
\hline Constructive coping & & & $1.24(0.93,1.66)$ & $1.26(0.94,1.68)$ \\
\hline Non-constructive coping & & & $1.75(1.30,2.36)$ & $1.67(1.23,2.28)$ \\
\hline
\end{tabular}

$\mathrm{AOR}=$ adjusted odds ratio $\mathrm{Cl}=$ confidence interval

aBolded odds ratios indicate a statistically significant association between the independent variable and suicide risk; odds ratios are adjusted for all other model variables bACE: Adverse Childhood Experiences

Includes 2 generations of boarding school attendance (parents, grandparents) and 3 generations of foster care/adoption (participant, parents, grandparents)

dParticipant only 
Appendix C.

Table 8. Multiple Stress-Coping Models (with Control Variables) of Suicide Risk for Montana-Wyoming American Indian Tribal College Students: $2012-2013$

\begin{tabular}{|c|c|c|c|c|c|}
\hline & $\operatorname{AOR}^{a}(95 \% \mathrm{Cl})$ & $\mathrm{AOR}^{a}(95 \% \mathrm{Cl})$ & $\mathrm{AOR}^{a}(95 \% \mathrm{Cl})$ & $\mathrm{AOR}^{a}(95 \% \mathrm{Cl})$ & $\mathrm{AOR}^{a}(95 \% \mathrm{Cl})$ \\
\hline & $\begin{array}{c}\text { Model } 1 \\
n=157 \text { AIC }=1485\end{array}$ & $\begin{array}{c}\text { Model } 2 \\
n=160 \text { AIC = } 1508\end{array}$ & $\begin{array}{c}\text { Model } 3 \\
n=154 \text { AIC }=1493\end{array}$ & $\begin{array}{c}\text { Model } 4 \\
n=151 \mathrm{AIC}=1422\end{array}$ & $\begin{array}{c}\text { Model } 5 \\
n=160 \mathrm{AIC}=1519\end{array}$ \\
\hline $\mathrm{ACE}^{\mathrm{b}}$ & $2.68(1.34,5.35)$ & $3.01(1.51,6.00)$ & $2.49(1.26,4.94)$ & $3.02(1.48,6.16)$ & $2.85(1.44,5.66)$ \\
\hline Traumatic events inventory & $2.04(0.86,4.85)$ & $3.35(1.38,8.12)$ & $2.53(1.09,5.89)$ & $2.69(1.08,6.72)$ & $2.43(1.03,5.72)$ \\
\hline Parental control (mom) & $1.27(1.04,1.55)$ & $1.26(1.03,1.53)$ & $1.27(1.04,1.54)$ & $1.26(1.03,1.53)$ & $1.26(1.04,1.53)$ \\
\hline Discrimination & $1.04(0.92,1.18)$ & $1.00(0.88,1.14)$ & $1.07(0.95,1.22)$ & $1.05(0.92,1.21)$ & $1.05(0.93,1.19)$ \\
\hline Historical loss & $1.09(0.96,1.24)$ & $1.10(0.97,1.25)$ & $1.10(0.98,1.25)$ & $1.19(1.04,1.36)$ & $1.10(0.97,1.24)$ \\
\hline Generational out-of-home placement ${ }^{c}$ & $0.95(0.87,1.03)$ & $0.95(0.87,1.04)$ & $0.93(0.85,1.01)$ & $0.89(0.81,0.98)$ & $0.94(0.86,1.02)$ \\
\hline Boarding school attendance ${ }^{d}$ & $0.38(0.23,0.62)$ & $0.52(0.33,0.82)$ & $0.46(0.30,0.71)$ & $0.55(0.35,0.87)$ & $0.45(0.29,0.69)$ \\
\hline Social Support & $0.90(0.82,0.98)$ & $0.88(0.80,0.96)$ & $0.91(0.83,1.00)$ & $0.88(0.80,0.97)$ & $0.89(0.82,0.98)$ \\
\hline Self-esteem & $0.82(0.59,1.13)$ & $0.89(0.65,1.23)$ & $0.87(0.63,1.20)$ & $0.84(0.60,1.17)$ & $0.90(0.65,1.23)$ \\
\hline Spirituality & $1.32(1.14,1.52)$ & $1.45(1.25,1.70)$ & $1.29(1.12,1.49)$ & $1.35(1.16,1.56)$ & $1.33(1.16,1.54)$ \\
\hline Resilience & $1.19(1.00,1.42)$ & $1.29(1.07,1.56)$ & $1.17(0.98,1.39)$ & $1.22(1.01,1.47)$ & $1.18(0.99,1.41)$ \\
\hline Gratitude & $0.74(0.63,0.87)$ & $0.69(0.59,0.81)$ & $0.75(0.64,0.87)$ & $0.75(0.64,0.87)$ & $0.75(0.64,0.87)$ \\
\hline American Indian identity & $1.06(0.90,1.26)$ & $1.05(0.90,1.24)$ & $1.00(0.84,1.18)$ & $1.03(0.86,1.22)$ & $1.04(0.88,1.23)$ \\
\hline Depressive symptoms & $1.30(0.95,1.80)$ & $1.04(0.75,1.43)$ & $1.15(0.84,1.56)$ & $1.10(0.79,1.54)$ & $1.17(0.86,1.60)$ \\
\hline Constructive coping & $1.33(0.99,1.78)$ & $1.11(0.82,1.50)$ & $1.23(0.92,1.64)$ & $1.21(0.89,1.63)$ & $1.22(0.91,1.64)$ \\
\hline Non-constructive coping & $1.44(1.04,2.00)$ & $1.81(1.33,2.48)$ & $1.51(1.10,2.08)$ & $1.59(1.12,2.26)$ & $1.71(1.25,2.34)$ \\
\hline Gender & $1.19(0.89,1.60)$ & & & & \\
\hline Age & & $0.83(0.75,0.92)$ & & & \\
\hline Current income & & & $0.92(0.86,0.99)$ & & \\
\hline Mother's Education & & & & $1.35(0.99,1.84)$ & \\
\hline Childhood home ${ }^{e}$ & & & & & $1.25(0.84,1.87)$ \\
\hline
\end{tabular}

$\mathrm{AOR}=$ adjusted odds ratio; $\mathrm{Cl}=$ confidence interval

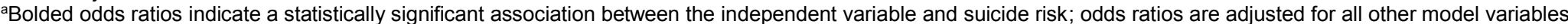
${ }^{b} \mathrm{ACE}:$ Adverse Childhood Experiences

Includes 2 generations of boarding school attendance (parents, grandparents) and 3 generations of foster care/adoption (participant, parents, grandparents)

PParticipant only

${ }^{\mathrm{e}} \mathrm{Grew}$ up on or near a reservation vs. urban area/other 
Appendix C.

Recruitment Flyer.

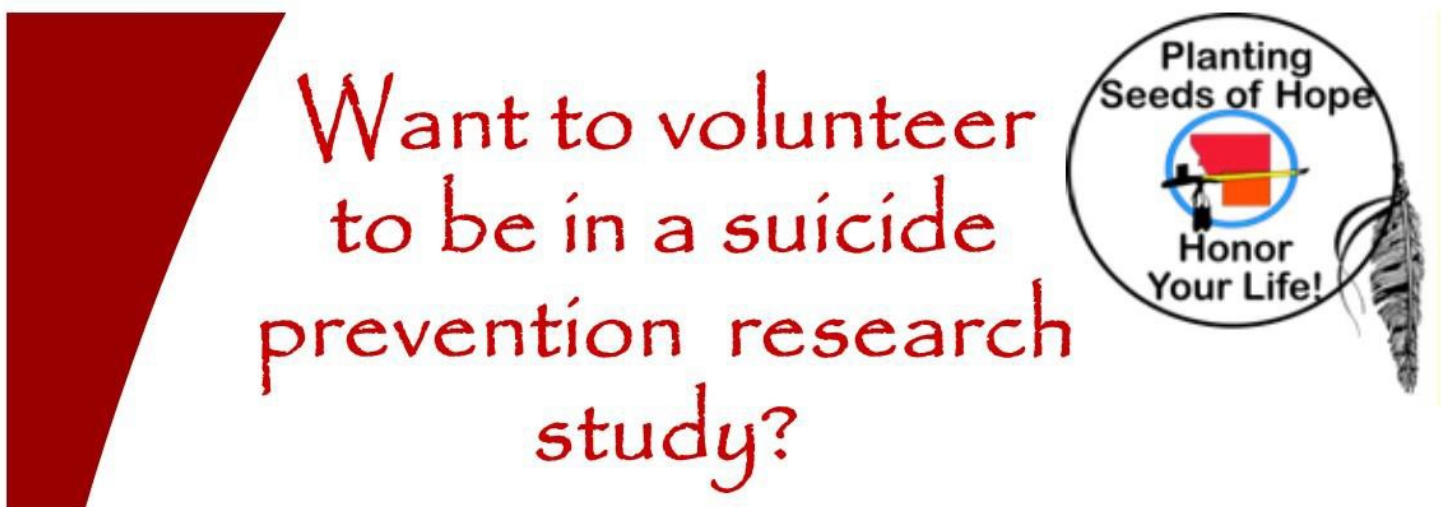

Suicide and Resilience in American Indian Tribal College Students: The Role of Enculturation on Psychological Well-Being

Are you American Indian?

Are you currently attending a Montana or Wyoming Tribal College? Are you 18 or Over?

If you answered YES to all 3 questions, then you are eligible to participate!

The purpose of the research is to see if there are links between culturallyunique life stress, coping, and suicide ideation/attempts in American Indian Tribal college students to help develop prevention programs.

The study:

- Uses an anonymous, web-based survey.

- Takes place during a 3 week time-period during Fall 2012.

- Will take you about 30-40 minutes to finish.

For your time:

- You will be entered in a drawing for an item valued at $\sim \$ 150$.

\section{West VrginiaUniversitye}

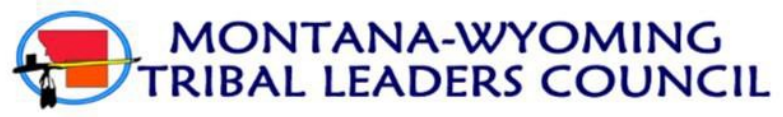

The study is a collaboration between the Montana Wyoming Tribal Leaders Council's Planting Seeds of Hope Youth Suicide Prevention Project and West Virginia University.

For More Information Contact:

Karen Manzo, Co-PI, WVU Jera Stewart, Co-PI, MTWYTLC Leon Rattler, Co-PI, MTWYTLC Arlana Farwell, Co-PI, MTWYTLC kamanzo@hsc.wvu.edu jstewart@mtwytlc.com Irattler@mtwytlc.com afarwell@mtwytlc.com
304-376-8486

406-252-2550

406-252-2550

406-252-2550 


\section{Appendix C.}

\section{Abridged Stress-Coping Survey.}

1. First, please tell us the name of the Tribal College you are currently attending.

Now, we'll start with questions that ask things about you now and when you were growing up, some family history, and questions about education and employment.

2. What is your age group?

O $18-21$ yrs

22-24 yrs

O 25-34 yrs

O $35-44$ yrs

O $\quad 45-54$ yrs

O 55 yrs and over

3. What is your gender?

O Male

O Female

4. What is your race/ethnic group? (Please check all that apply.)

a American Indian/Alaska Native (Please enter the name of your Tribe.)

口 White

ㅁ Black/African American

ㄱian

- Hispanic

ㅁ Other (Please describe.)

5. Where did you spend most of your childhood?

O On a reservation (Please enter the name of the Reservation below.)

Near a reservation (within 100 miles)

O In an urban area

Other (Please describe below.) 
6 . What is your current annual household income?

Less than $\$ 1,000$

O $\$ 1,000-4,999$

O $\$ 5,000-9,999$

O $\$ 10,000-19,999$

O $\$ 20,000-29,999$

O $\$ 30,000-39,999$

O $\$ 40,000-49,999$

O $\$ 50,000$ or more

7. What's the highest year in school your MOTHER completed?

O Grade school

O Some high school

O GED

12th grade

O Some college

O 2 year college degree

O 4 year college degree

- Graduate degree

O Don't know

8. What's the highest year in school your FATHER completed?

Grade school

O Some high school

O GED

O 12th grade

O Some college

O 2 year college degree

O 4 year college degree

O Graduate degree

O Don't know 
9. When you were growing up, how often did you have...

\begin{tabular}{|l|l|l|l|l|l|}
\hline & $\begin{array}{l}\text { Almost } \\
\text { Always }\end{array}$ & Often & Sometimes & Almost Never & Never \\
\hline Enough food to eat & O & O & O & O & O \\
\hline Enough clothes & O & O & O & O & O \\
\hline A decent place to live & O & O & O & O & 0 \\
\hline
\end{tabular}

10. When you were growing up, how often...

\begin{tabular}{|l|l|l|l|l|l|}
\hline & $\begin{array}{l}\text { Almost } \\
\text { Always }\end{array}$ & Often & Sometimes & $\begin{array}{l}\text { Almost } \\
\text { Never }\end{array}$ & Never \\
\hline Was your mother treated violently & $\mathrm{O}$ & $\mathrm{O}$ & $\mathrm{O}$ & $\mathrm{O}$ & $\mathrm{O}$ \\
\hline Were your emotional needs met & $\mathrm{O}$ & $\mathrm{O}$ & $\mathrm{O}$ & $\mathrm{O}$ & $\mathrm{O}$ \\
\hline
\end{tabular}

11. When you were growing up....

\begin{tabular}{|l|l|l|}
\hline & Yes & No \\
\hline $\begin{array}{l}\text { Did you live with someone who had a problem with drugs or } \\
\text { alcohol }\end{array}$ & O & O \\
\hline Did you live with someone who had a mental problem & O & O \\
\hline Did your parent's divorce or separate & O & O \\
\hline Did you live with a handicapped person & O & O \\
\hline
\end{tabular}

12. Did you go to boarding school?

O Yes

O No

13. Were you placed in foster care as a child?

○ Yes

No

14. Were you adopted out as a child?

O Yes

No 
15. Did either of your PARENTS or GRANDPARENTS attend a boarding school?

\begin{tabular}{|c|c|c|c|}
\hline \multicolumn{4}{|c|}{ | } \\
\hline & Yes & No & Don't Know \\
\hline Mother & $\mathrm{O}$ & $\mathrm{O}$ & $\mathrm{O}$ \\
\hline Father & $\mathrm{O}$ & $\mathrm{O}$ & $\mathrm{O}$ \\
\hline $\begin{array}{l}\text { Maternal } \\
\text { Grandmother }\end{array}$ & $\mathrm{O}$ & $\mathrm{O}$ & $\mathrm{O}$ \\
\hline $\begin{array}{l}\text { Maternal } \\
\text { Grandfather }\end{array}$ & $\mathrm{O}$ & $\mathrm{O}$ & $\mathrm{O}$ \\
\hline $\begin{array}{l}\text { Paternal } \\
\text { Grandmother }\end{array}$ & $\mathrm{O}$ & $\mathrm{O}$ & $\mathrm{O}$ \\
\hline $\begin{array}{l}\text { Paternal } \\
\text { Grandfather }\end{array}$ & $\mathrm{O}$ & $\mathrm{O}$ & O \\
\hline
\end{tabular}

16. Were either of your PARENTS or GRANDPARENTS placed in foster care or adopted out?

\begin{tabular}{|l|l|l|l|}
\hline & Yes & No & Don't Know \\
\hline Mother & 0 & 0 & 0 \\
\hline Father & 0 & 0 & 0 \\
\hline $\begin{array}{l}\text { Maternal } \\
\text { Grandmother }\end{array}$ & 0 & 0 & 0 \\
$\begin{array}{l}\text { Maternal } \\
\text { Grandfather }\end{array}$ & 0 & 0 & 0 \\
\hline $\begin{array}{l}\text { Paternal } \\
\text { Grandmother } \\
\text { Paternal } \\
\text { Grandfather }\end{array}$ & 0 & 0 & 0 \\
\hline
\end{tabular}

17. Have any of your PARENTS/CAREGIVERS...

\begin{tabular}{|l|l|l|l|}
\hline & Yes & No & Don't know \\
\hline Been arrested & O & O & O \\
\hline Spent time in jail & O & O & 0 \\
\hline
\end{tabular}


18. This set of questions asks about experiences that may have happened to you in your day-to-day life. In your DAY-TO-DAY LIFE, how often do any of the following things happen to you?

\begin{tabular}{|c|c|c|c|c|c|c|}
\hline & $\begin{array}{l}\text { Almost } \\
\text { Everyday }\end{array}$ & $\begin{array}{l}\text { At Least } \\
\text { Once a } \\
\text { Week }\end{array}$ & $\begin{array}{l}\text { A Few } \\
\text { Times a } \\
\text { Month }\end{array}$ & $\begin{array}{l}\text { A Few } \\
\text { Times a } \\
\text { Year }\end{array}$ & $\begin{array}{l}\text { Less Than } \\
\text { Once a } \\
\text { Year }\end{array}$ & Never \\
\hline $\begin{array}{l}\text { You are treated with less courtesy than } \\
\text { other people are. }\end{array}$ & $\mathrm{O}$ & $\mathrm{O}$ & $\mathrm{O}$ & $\mathrm{O}$ & $\mathrm{O}$ & $\mathrm{O}$ \\
\hline $\begin{array}{l}\text { You are treated with less respect than other } \\
\text { people are. }\end{array}$ & $\mathrm{O}$ & $\mathrm{O}$ & $\mathrm{O}$ & $\mathrm{O}$ & $\mathrm{O}$ & $\mathrm{O}$ \\
\hline $\begin{array}{l}\text { You receive poorer service than other } \\
\text { people at restaurants or stores. }\end{array}$ & $\mathrm{O}$ & $\mathrm{O}$ & $\mathrm{O}$ & $\mathrm{O}$ & $\mathrm{O}$ & $\mathrm{O}$ \\
\hline $\begin{array}{l}\text { People act as if they think you are not } \\
\text { smart. }\end{array}$ & $\mathrm{O}$ & $\mathrm{O}$ & $\mathrm{O}$ & $\mathrm{O}$ & $\mathrm{O}$ & $\mathrm{O}$ \\
\hline People act as if they are afraid of you. & $\mathrm{O}$ & $\mathrm{O}$ & $\mathrm{O}$ & $\mathrm{O}$ & $\mathrm{O}$ & $\mathrm{O}$ \\
\hline $\begin{array}{l}\text { People act as if they think you are } \\
\text { dishonest. }\end{array}$ & $\mathrm{O}$ & $\mathrm{O}$ & O & O & $\mathrm{O}$ & $\mathrm{O}$ \\
\hline People act as if they're better than you are. & $\mathrm{O}$ & $\mathrm{O}$ & $\mathrm{O}$ & O & $\mathrm{O}$ & $\mathrm{O}$ \\
\hline You are called names or insulted. & $\mathrm{O}$ & $\mathrm{O}$ & $\mathrm{O}$ & $\mathrm{O}$ & $\mathrm{O}$ & $\mathrm{O}$ \\
\hline You are threatened or harassed. & $\mathrm{O}$ & $\mathrm{O}$ & $\mathrm{O}$ & $\mathrm{O}$ & $\mathrm{O}$ & $\mathrm{O}$ \\
\hline
\end{tabular}

19. The next set of questions pertains to cultural losses. How often do you think about the following...

\begin{tabular}{|c|c|c|c|c|c|c|}
\hline & Never & $\begin{array}{l}\text { Yearly/Special } \\
\text { Times }\end{array}$ & Monthly & Weekly & Daily & $\begin{array}{l}\text { Several } \\
\text { Times a } \\
\text { Day }\end{array}$ \\
\hline Loss of our land & $\mathrm{O}$ & $\mathrm{O}$ & $\mathrm{O}$ & $\mathrm{O}$ & $\mathrm{O}$ & $\mathrm{O}$ \\
\hline Loss of our language & $\mathrm{O}$ & $\mathrm{O}$ & $\mathrm{O}$ & $\mathrm{O}$ & $\mathrm{O}$ & $\mathrm{O}$ \\
\hline Losing our traditional spiritual ways & $\mathrm{O}$ & $\mathrm{O}$ & $\mathrm{O}$ & $\mathrm{O}$ & $\mathrm{O}$ & $\mathrm{O}$ \\
\hline $\begin{array}{l}\text { Loss of our family ties because of boarding } \\
\text { schools }\end{array}$ & $\mathrm{O}$ & O & $\mathrm{O}$ & $\mathrm{O}$ & $\mathrm{O}$ & $\mathrm{O}$ \\
\hline $\begin{array}{l}\text { Loss of families from the reservation to } \\
\text { government relocation }\end{array}$ & $\mathrm{O}$ & $\mathrm{O}$ & $\mathrm{O}$ & $\mathrm{O}$ & $\mathrm{O}$ & $\mathrm{O}$ \\
\hline $\begin{array}{l}\text { Loss of self-respect from poor treatment by } \\
\text { government officials }\end{array}$ & $\mathrm{O}$ & $\mathrm{O}$ & $\mathrm{O}$ & O & $\mathrm{O}$ & $\mathrm{O}$ \\
\hline Loss of trust in Whites from broken treaties & $\mathrm{O}$ & $\mathrm{O}$ & $\mathrm{O}$ & $\mathrm{O}$ & $\mathrm{O}$ & $\mathrm{O}$ \\
\hline Losing our culture & $\mathrm{O}$ & $\mathrm{O}$ & $\mathrm{O}$ & $\mathrm{O}$ & $\mathrm{O}$ & $\mathrm{O}$ \\
\hline $\begin{array}{l}\text { Losses from the effects of alcoholism on our } \\
\text { people }\end{array}$ & $\mathrm{O}$ & $\mathrm{O}$ & $\mathrm{O}$ & $\mathrm{O}$ & $\mathrm{O}$ & $\mathrm{O}$ \\
\hline $\begin{array}{l}\text { Loss of respect by our children and } \\
\text { grandchildren for elders }\end{array}$ & $\mathrm{O}$ & $\mathrm{O}$ & $\mathrm{O}$ & $\mathrm{O}$ & $\mathrm{O}$ & $\mathrm{O}$ \\
\hline Loss of our people through early death & $\mathrm{O}$ & $\mathrm{O}$ & $\mathrm{O}$ & $\mathrm{O}$ & $\mathrm{O}$ & $\mathrm{O}$ \\
\hline $\begin{array}{l}\text { Loss of respect by our children for traditional } \\
\text { ways }\end{array}$ & $\mathrm{O}$ & O & $\mathrm{O}$ & O & $\mathrm{O}$ & $\mathrm{O}$ \\
\hline
\end{tabular}


20. The following questions ask about those in your life who provide you with help or support. Each question has two parts. For the first part, check all those, excluding yourself, who you can count on for help or support in the manner described. Then check how satisfied you are with the overall support you have. If you have no support for a question, check the words "No One", but still rate your level of satisfaction.

\begin{tabular}{|c|c|c|c|c|c|c|c|c|c|c|}
\hline & \multicolumn{10}{|c|}{ Those in your life who provide help or support } \\
\hline & Parent & $\begin{array}{l}\text { Grand } \\
\text { parent }\end{array}$ & Sibling & $\begin{array}{l}\text { Other } \\
\text { Relative }\end{array}$ & Friend & $\begin{array}{l}\text { God/ } \\
\text { Spiritual } \\
\text { Being }\end{array}$ & $\begin{array}{l}\text { Spiritual } \\
\text { Elder }\end{array}$ & Clergy & $\begin{array}{l}\text { No } \\
\text { One }\end{array}$ & Other \\
\hline $\begin{array}{l}\text { Who can you } \\
\text { really count on to } \\
\text { be dependable } \\
\text { when you need } \\
\text { help? }\end{array}$ & $\square$ & $\square$ & $\square$ & $\square$ & $\square$ & $\square$ & $\square$ & $\square$ & $\square$ & $\square$ \\
\hline $\begin{array}{l}\text { Who can you } \\
\text { really count on to } \\
\text { help you feel more } \\
\text { relaxed when you } \\
\text { are under } \\
\text { pressure or } \\
\text { tense? }\end{array}$ & $\square$ & $\square$ & $\square$ & $\square$ & $\square$ & $\square$ & $\square$ & $\square$ & $\square$ & $\square$ \\
\hline $\begin{array}{l}\text { Who accepts you } \\
\text { totally, including } \\
\text { both your worst } \\
\text { and your best } \\
\text { points? }\end{array}$ & $\square$ & $\square$ & $\square$ & $\square$ & $\square$ & $\square$ & $\square$ & $\square$ & $\square$ & $\square$ \\
\hline $\begin{array}{l}\text { Who can you } \\
\text { really count on to } \\
\text { care about you } \\
\text { regardless of what } \\
\text { is happening to } \\
\text { you? }\end{array}$ & $\square$ & $\square$ & $\square$ & $\square$ & $\square$ & $\square$ & $\square$ & $\square$ & 口 & 口 \\
\hline $\begin{array}{l}\text { Who can you } \\
\text { really count on to } \\
\text { help you feel } \\
\text { better when you } \\
\text { are feeling } \\
\text { generally down-in- } \\
\text { the-dumps? }\end{array}$ & 口 & 口 & 口 & 口 & 口 & 口 & 口 & 口 & 口 & 口 \\
\hline $\begin{array}{l}\text { Who can you } \\
\text { count on to } \\
\text { console you when } \\
\text { you are very } \\
\text { upset? }\end{array}$ & 口 & 口 & 口 & 口 & 口 & 口 & 口 & 口 & 口 & 口 \\
\hline
\end{tabular}

21. The next set of questions is about your ethnic identity. Please answer for both columns (White or Anglo; American Indian) if they both pertain to you.

\begin{tabular}{|c|c|c|c|c|c|c|c|c|c|c|}
\hline & \multicolumn{5}{|c|}{ The American Indian way of life } & \multicolumn{5}{|c|}{ The White or Anglo way of life } \\
\hline & $\begin{array}{l}\text { Not } \\
\text { at All }\end{array}$ & $\begin{array}{l}\text { Not } \\
\text { Much }\end{array}$ & Somewhat & A Lot & $\begin{array}{l}\text { Very } \\
\text { Much }\end{array}$ & $\begin{array}{l}\text { Not } \\
\text { at All }\end{array}$ & $\begin{array}{l}\text { Not } \\
\text { Much }\end{array}$ & Somewhat & A Lot & $\begin{array}{l}\text { Very } \\
\text { Much }\end{array}$ \\
\hline $\begin{array}{l}\text { Do you live by } \\
\text { the following }\end{array}$ & $\mathrm{O}$ & $\mathrm{O}$ & $\mathrm{O}$ & $\mathrm{O}$ & $\mathrm{O}$ & $\mathrm{O}$ & $\mathrm{O}$ & $\mathrm{O}$ & $\mathrm{O}$ & $\mathrm{O}$ \\
\hline $\begin{array}{l}\text { Are you/will you } \\
\text { be a success in }\end{array}$ & $\mathrm{O}$ & $\mathrm{O}$ & O & $\mathrm{O}$ & O & $\mathrm{O}$ & O & O & O & $\mathrm{O}$ \\
\hline $\begin{array}{l}\text { Does your family } \\
\text { live by }\end{array}$ & $\mathrm{O}$ & $\mathrm{O}$ & $\mathrm{O}$ & $\mathrm{O}$ & $\mathrm{O}$ & $\mathrm{O}$ & $\mathrm{O}$ & $\mathrm{O}$ & $\mathrm{O}$ & $\mathrm{O}$ \\
\hline $\begin{array}{l}\text { Is your family a } \\
\text { success in }\end{array}$ & $\mathrm{O}$ & $\mathrm{O}$ & O & $\mathrm{O}$ & $\mathrm{O}$ & $\mathrm{O}$ & $\mathrm{O}$ & O & $\mathrm{O}$ & $\mathrm{O}$ \\
\hline
\end{tabular}


22. Some families haves special activities or traditions (such as holiday parties, special meals, religious activities, trips, or visits). In your family, how many of the activities or traditions are based on...

\begin{tabular}{|l|l|l|l|l|l|}
\hline & $\begin{array}{l}\text { Very } \\
\text { Much }\end{array}$ & A Lot & Somewhat & Not Much & Not at All \\
\hline White Culture & O & O & O & O & O \\
\hline $\begin{array}{l}\text { American Indian } \\
\text { Culture }\end{array}$ & O & O & O & O & O \\
\hline
\end{tabular}

23. How involved are you in...

\begin{tabular}{|l|l|l|l|l|l|}
\hline & $\begin{array}{l}\text { Very } \\
\text { Much }\end{array}$ & A Lot & Somewhat & Not Much & Not at All \\
\hline White Traditions \& Beliefs & O & O & O & O & O \\
\hline $\begin{array}{l}\text { American Indian Traditions \& } \\
\text { Beliefs }\end{array}$ & O & O & O & $\bigcirc$ & $\bigcirc$ \\
\hline
\end{tabular}

24. How important it is for you to follow religious or spiritual beliefs that are based on...

\begin{tabular}{|c|c|c|c|c|c|}
\hline & $\begin{array}{l}\text { Not at all } \\
\text { Important }\end{array}$ & $\begin{array}{l}\text { Very } \\
\text { Unimportant }\end{array}$ & $\begin{array}{l}\text { Neither Important } \\
\text { nor Unimportant }\end{array}$ & $\begin{array}{l}\text { Very } \\
\text { Important }\end{array}$ & $\begin{array}{l}\text { Extremely } \\
\text { Important }\end{array}$ \\
\hline Christian Beliefs & $\mathrm{O}$ & $\mathrm{O}$ & $\mathrm{O}$ & $\mathrm{O}$ & $\mathrm{O}$ \\
\hline $\begin{array}{l}\text { Traditional Indian } \\
\text { Beliefs }\end{array}$ & $\mathrm{O}$ & $\mathrm{O}$ & $\bigcirc$ & $\mathrm{O}$ & $\mathrm{O}$ \\
\hline
\end{tabular}

25. What languages were spoken in your home when you were growing up?

\begin{tabular}{|l|l|l|l|l|l|l|}
\hline & Never & Rarely & Sometimes & Often & $\begin{array}{l}\text { Almost } \\
\text { Always }\end{array}$ & Exclusively \\
\hline English & O & O & O & O & O & O \\
\hline Tribal Language & O & O & O & $\bigcirc$ & $\bigcirc$ & $\bigcirc$ \\
\hline
\end{tabular}

26. How important is spirituality in your life?

O Extremely Important

Very Important

Neither Important nor Unimportant

Not Very Important

Not at all Important

26a. How often do you spend time on religious or spiritual practices?

Everyday or Almost Everyday

Several Times a Month

From Time to Time, Occasionally

V Very Rarely

Not at All 
26b. How often do you seek comfort or guidance through religious or spiritual means?

O Never

O Rarely

Sometimes

O Quite Often

O Very Often

27. The next set of questions relates to your experiences with your parents/caregivers when you were growing up. The same questions are asked for your MOTHER/FEMALE CAREGIVER and FATHER/MALE CAREGIVER. Use NA

Not Applicable) where needed. DURING YOUR FIRST 16 YEARS, how 'true' are the following statements about your CAREGIVER's behavior towards you?

\begin{tabular}{|c|c|c|c|c|c|c|c|c|c|c|}
\hline & \multicolumn{5}{|c|}{ MOTHER / FEMALE CAREGIVER } & \multicolumn{5}{|c|}{ | FATHER / MALE CAREGIVER } \\
\hline & $\begin{array}{l}N \\
A\end{array}$ & $\begin{array}{l}\text { Not } \\
\text { True } \\
\text { at All }\end{array}$ & $\begin{array}{l}\text { Slightly } \\
\text { True }\end{array}$ & $\begin{array}{l}\text { Moderatel } \\
\text { y True }\end{array}$ & $\begin{array}{l}\text { Extremel } \\
\text { y True }\end{array}$ & NA & $\begin{array}{l}\text { Not } \\
\text { True } \\
\text { at All }\end{array}$ & $\begin{array}{l}\text { Slightly } \\
\text { True }\end{array}$ & $\begin{array}{l}\text { Moderatel } \\
\text { y True }\end{array}$ & $\begin{array}{l}\text { Extremel } \\
\text { y True }\end{array}$ \\
\hline $\begin{array}{l}\text { Overprotective } \\
\text { of me }\end{array}$ & O & $\mathrm{O}$ & 0 & O & 0 & $\mathrm{O}$ & $\mathrm{O}$ & 0 & 0 & O \\
\hline $\begin{array}{l}\text { Verbally } \\
\text { abusive of me }\end{array}$ & $\mathrm{O}$ & 0 & 0 & 0 & 0 & 0 & 0 & O & 0 & 0 \\
\hline $\begin{array}{l}\text { Over } \\
\text { controlling of } \\
\text { me }\end{array}$ & O & 0 & O & 0 & 0 & 0 & O & O & 0 & 0 \\
\hline $\begin{array}{l}\text { Tried to make } \\
\text { me feel guilty }\end{array}$ & 0 & 0 & 0 & 0 & $\mathrm{O}$ & 0 & 0 & 0 & 0 & 0 \\
\hline Ignored me & 0 & 0 & O & 0 & 0 & 0 & 0 & 0 & 0 & 0 \\
\hline Critical of me & 0 & 0 & 0 & 0 & 0 & 0 & 0 & 0 & 0 & 0 \\
\hline $\begin{array}{l}\text { Unpredictable } \\
\text { towards me }\end{array}$ & 0 & 0 & 0 & 0 & 0 & 0 & 0 & O & 0 & 0 \\
\hline Uncaring of me & 0 & 0 & 0 & 0 & 0 & 0 & 0 & 0 & 0 & 0 \\
\hline $\begin{array}{l}\text { Physically } \\
\text { violent or } \\
\text { abusive of me }\end{array}$ & O & 0 & $\mathrm{O}$ & 0 & 0 & 0 & $\mathrm{O}$ & $\mathrm{O}$ & 0 & 0 \\
\hline Rejecting of me & O & 0 & 0 & 0 & 0 & 0 & 0 & 0 & 0 & 0 \\
\hline $\begin{array}{l}\text { Left me on my } \\
\text { own a lot }\end{array}$ & 0 & 0 & O & 0 & 0 & 0 & 0 & O & 0 & 0 \\
\hline $\begin{array}{l}\text { Would forget } \\
\text { about me }\end{array}$ & 0 & 0 & 0 & 0 & 0 & 0 & 0 & O & 0 & 0 \\
\hline $\begin{array}{l}\text { Was } \\
\text { uninterested in } \\
\text { me }\end{array}$ & O & 0 & O & O & 0 & $\mathrm{O}$ & O & O & $\mathrm{O}$ & O \\
\hline $\begin{array}{l}\text { Made me feel } \\
\text { in danger }\end{array}$ & 0 & 0 & O & 0 & 0 & 0 & O & O & 0 & 0 \\
\hline $\begin{array}{l}\text { Made me feel } \\
\text { unsafe }\end{array}$ & 0 & 0 & 0 & 0 & 0 & 0 & 0 & O & 0 & O \\
\hline
\end{tabular}


28. These questions list various attitudes and behaviors of parents/caregivers. As you remember your PARENT/CAREGIVER in YOUR FIRST 16 YEARS would you please check the most appropriate box next to each question? DURING YOUR FIRST 16 YEARS, how like this were your PARENT/CAREGIVERS...

\begin{tabular}{|c|c|c|c|c|c|c|c|c|c|c|}
\hline & \multicolumn{5}{|c|}{ MOTHER / FEMALE CAREGIVER } & \multicolumn{5}{|c|}{ FATHER / MALE CAREGIVER } \\
\hline & NA & $\begin{array}{l}\text { Very } \\
\text { Like }\end{array}$ & $\begin{array}{l}\text { Moderately } \\
\text { Like }\end{array}$ & $\begin{array}{l}\text { Moderately } \\
\text { Unlike }\end{array}$ & $\begin{array}{l}\text { Very } \\
\text { Unlike }\end{array}$ & NA & $\begin{array}{l}\text { Very } \\
\text { Like }\end{array}$ & $\begin{array}{l}\text { Moderately } \\
\text { Like }\end{array}$ & $\begin{array}{l}\text { Moderately } \\
\text { Unlike }\end{array}$ & $\begin{array}{l}\text { Very } \\
\text { Unlike }\end{array}$ \\
\hline $\begin{array}{l}\text { Appeared to } \\
\text { understand } \\
\text { my problems } \\
\text { and worries }\end{array}$ & $\mathrm{O}$ & $\mathrm{O}$ & $\mathrm{O}$ & $\bigcirc$ & $\mathrm{O}$ & $\bigcirc$ & $\bigcirc$ & $\mathrm{O}$ & $\mathrm{O}$ & $\mathrm{O}$ \\
\hline $\begin{array}{l}\text { Could confide } \\
\text { in them about } \\
\text { things that } \\
\text { were } \\
\text { bothering me }\end{array}$ & $\mathrm{O}$ & O & O & $\mathrm{O}$ & $\mathrm{O}$ & $\mathrm{O}$ & $\mathrm{O}$ & $\bigcirc$ & $\mathrm{O}$ & $\mathrm{O}$ \\
\hline $\begin{array}{l}\text { Gave me time } \\
\text { and attention } \\
\text { when I } \\
\text { needed it }\end{array}$ & $\mathrm{O}$ & $\mathrm{O}$ & $\mathrm{O}$ & $\bigcirc$ & $\bigcirc$ & $\mathrm{O}$ & $\bigcirc$ & $\mathrm{O}$ & $\bigcirc$ & $\mathrm{O}$ \\
\hline $\begin{array}{l}\text { Gave me love } \\
\text { and affection }\end{array}$ & $\mathrm{O}$ & O & O & $\mathrm{O}$ & $\mathrm{O}$ & 0 & $\mathrm{O}$ & 0 & $\mathrm{O}$ & $\mathrm{O}$ \\
\hline $\begin{array}{l}\text { Appeared to } \\
\text { put effort into } \\
\text { watching over } \\
\text { me and } \\
\text { making sure I } \\
\text { had a good } \\
\text { upbringing }\end{array}$ & $\mathrm{O}$ & $\mathrm{O}$ & $\mathrm{O}$ & $\mathrm{O}$ & $\mathrm{O}$ & $\mathrm{O}$ & $\mathrm{O}$ & $\bigcirc$ & $\mathrm{O}$ & $\mathrm{O}$ \\
\hline $\begin{array}{l}\text { Had strict } \\
\text { rules for me }\end{array}$ & $\mathrm{O}$ & 0 & O & $\mathrm{O}$ & 0 & 0 & $\mathrm{O}$ & $\mathrm{O}$ & $\mathrm{O}$ & $\mathrm{O}$ \\
\hline $\begin{array}{l}\text { Was } \\
\text { consistent } \\
\text { about the } \\
\text { rules for me }\end{array}$ & $\mathrm{O}$ & $\mathrm{O}$ & $\mathrm{O}$ & $\mathrm{O}$ & $\mathrm{O}$ & $\mathrm{O}$ & $\mathrm{O}$ & $\mathrm{O}$ & $\mathrm{O}$ & $\mathrm{O}$ \\
\hline $\begin{array}{l}\text { Expected me } \\
\text { to do my best } \\
\text { in everything I } \\
\text { did }\end{array}$ & $\mathrm{O}$ & 0 & 0 & $\mathrm{O}$ & $\mathrm{O}$ & $\mathrm{O}$ & $\mathrm{O}$ & $\mathrm{O}$ & 0 & $\mathrm{O}$ \\
\hline $\begin{array}{l}\text { Stopped me } \\
\text { from doing } \\
\text { things that } \\
\text { other kids my } \\
\text { age were } \\
\text { allowed to do }\end{array}$ & $\mathrm{O}$ & $\mathrm{O}$ & $\mathrm{O}$ & $\mathrm{O}$ & $\mathrm{O}$ & $\mathrm{O}$ & $\mathrm{O}$ & O & $\mathrm{O}$ & $\mathrm{O}$ \\
\hline $\begin{array}{l}\text { Was } \\
\text { overprotective } \\
\text { of me }\end{array}$ & $\mathrm{O}$ & 0 & O & $\mathrm{O}$ & 0 & O & $\mathrm{O}$ & O & $\mathrm{O}$ & $\mathrm{O}$ \\
\hline Babied me & $\mathrm{O}$ & $\mathrm{O}$ & $\mathrm{O}$ & $\mathrm{O}$ & $\mathrm{O}$ & $\mathrm{O}$ & $\mathrm{O}$ & $\mathrm{O}$ & $\mathrm{O}$ & $\mathrm{O}$ \\
\hline $\begin{array}{l}\text { Abandoned } \\
\text { me }\end{array}$ & $\mathrm{O}$ & $\mathrm{O}$ & $\mathrm{O}$ & $\mathrm{O}$ & $\mathrm{O}$ & 0 & $\mathrm{O}$ & 0 & 0 & $\mathrm{O}$ \\
\hline
\end{tabular}


29. How true is this statement for you? When I was growing up, I felt...

\begin{tabular}{|l|l|l|l|l|}
\hline & NA & $\begin{array}{l}\text { Not True } \\
\text { at All }\end{array}$ & $\begin{array}{l}\text { Slightly } \\
\text { True }\end{array}$ & $\begin{array}{l}\text { Moderately } \\
\text { True }\end{array}$ \\
\hline $\begin{array}{l}\text { Responsible for things my parents should be } \\
\text { doing. }\end{array}$ & O & O & O & O \\
\hline Like I was a parent to my parent. & O & O & $\bigcirc$ & $\bigcirc$ \\
\hline
\end{tabular}

30. Please answer the following questions about yourself.

\begin{tabular}{|c|c|c|c|c|}
\hline & $\begin{array}{l}\text { Strongly } \\
\text { Agree }\end{array}$ & Agree & Disagree & $\begin{array}{l}\text { Strongly } \\
\text { Disagree }\end{array}$ \\
\hline I feel that I have many good qualities. & O & $\mathrm{O}$ & $\mathrm{O}$ & O \\
\hline I feel that I am a failure. & O & $\mathrm{O}$ & O & $\bigcirc$ \\
\hline I take a positive attitude toward myself. & O & $\mathrm{O}$ & $\mathrm{O}$ & $\mathrm{O}$ \\
\hline I feel useless at times. & O & O & O & O \\
\hline $\begin{array}{l}\text { On the whole, I am satisfied with } \\
\text { myself. }\end{array}$ & $\mathrm{O}$ & $\mathrm{O}$ & $\mathrm{O}$ & $\mathrm{O}$ \\
\hline At times I think I am no good at all. & O & $\mathrm{O}$ & $\bigcirc$ & $\mathrm{O}$ \\
\hline
\end{tabular}

31. The next 10 questions relate to the amount of stress in your life. In the LAST MONTH, how often have you...

\begin{tabular}{|c|c|c|c|c|c|}
\hline & Never & $\begin{array}{l}\text { Almost } \\
\text { Never }\end{array}$ & Sometimes & $\begin{array}{l}\text { Fairly } \\
\text { Often }\end{array}$ & $\begin{array}{l}\text { Very } \\
\text { Often }\end{array}$ \\
\hline $\begin{array}{l}\text { been upset because of something that happened } \\
\text { unexpectedly }\end{array}$ & $\mathrm{O}$ & $\mathrm{O}$ & $\mathrm{O}$ & 0 & $\mathrm{O}$ \\
\hline $\begin{array}{l}\text { felt that you were unable to control the important } \\
\text { things in your life }\end{array}$ & $\mathrm{O}$ & $\mathrm{O}$ & $\mathrm{O}$ & O & $\mathrm{O}$ \\
\hline felt nervous and "stressed"? & O & O & $\mathrm{O}$ & O & O \\
\hline $\begin{array}{l}\text { felt confident about your ability to handle your } \\
\text { personal problems }\end{array}$ & $\mathrm{O}$ & $\mathrm{O}$ & $\mathrm{O}$ & O & $\mathrm{O}$ \\
\hline felt that things were going your way & $\mathrm{O}$ & $\mathrm{O}$ & $\mathrm{O}$ & $\bigcirc$ & $\mathrm{O}$ \\
\hline $\begin{array}{l}\text { found that you could not cope with all the things } \\
\text { that you had to do }\end{array}$ & 0 & 0 & 0 & O & $\mathrm{O}$ \\
\hline been able to control irritations in your life & O & $\mathrm{O}$ & O & O & O \\
\hline felt that you were on top of things & 0 & 0 & O & 0 & 0 \\
\hline $\begin{array}{l}\text { been angered because of things that happened } \\
\text { that were outside of your control }\end{array}$ & $\mathrm{O}$ & O & 0 & 0 & 0 \\
\hline $\begin{array}{l}\text { felt difficulties were piling up so high that you could } \\
\text { not overcome them }\end{array}$ & 0 & 0 & 0 & 0 & 0 \\
\hline
\end{tabular}


32. The next items deal with how you cope with stress in your life.

\begin{tabular}{|c|c|c|c|c|}
\hline & $\begin{array}{l}\text { I Don't Do } \\
\text { This at All }\end{array}$ & $\begin{array}{l}\text { I Do This } \\
\text { a Little Bit }\end{array}$ & $\begin{array}{l}\text { I Do This a } \\
\text { Medium Amount }\end{array}$ & $\begin{array}{l}\text { I Do This } \\
\text { a Lot }\end{array}$ \\
\hline I turn to work or other activities to take my mind off things. & $\mathrm{O}$ & $\mathrm{O}$ & $\mathrm{O}$ & $\mathrm{O}$ \\
\hline $\begin{array}{l}\text { I concentrate my efforts on doing something about the situation } \\
\text { I'm in. }\end{array}$ & O & O & $\mathrm{O}$ & O \\
\hline I say to myself "this isn't real." & $\mathrm{O}$ & $\mathrm{O}$ & $\mathrm{O}$ & $\mathrm{O}$ \\
\hline I use alcohol or other drugs to make myself feel better. & $\mathrm{O}$ & O & $\mathrm{O}$ & $\mathrm{O}$ \\
\hline I get emotional support from others. & $\mathrm{O}$ & $\mathrm{O}$ & $\mathrm{O}$ & $\mathrm{O}$ \\
\hline I give up trying to deal with it. & $\mathrm{O}$ & $\mathrm{O}$ & $\mathrm{O}$ & $\mathrm{O}$ \\
\hline I take action to try to make the situation better. & $\mathrm{O}$ & $\mathrm{O}$ & $\mathrm{O}$ & $\mathrm{O}$ \\
\hline I refuse to believe that it has happened. & $\mathrm{O}$ & $\mathrm{O}$ & $\mathrm{O}$ & $\mathrm{O}$ \\
\hline I say things to let my unpleasant feelings escape. & $\mathrm{O}$ & $\mathrm{O}$ & $\mathrm{O}$ & $\mathrm{O}$ \\
\hline I get help and advice from other people. & $\mathrm{O}$ & $\mathrm{O}$ & $\mathrm{O}$ & O \\
\hline I use alcohol or other drugs to help me get through it. & $\mathrm{O}$ & $\mathrm{O}$ & $\mathrm{O}$ & $\mathrm{O}$ \\
\hline I try to see it in a different light, to make it seem more positive. & $\mathrm{O}$ & $\mathrm{O}$ & $\mathrm{O}$ & $\mathrm{O}$ \\
\hline I criticize myself. & $\mathrm{O}$ & $\mathrm{O}$ & $\mathrm{O}$ & O \\
\hline I try to come up with a strategy about what to do. & $\mathrm{O}$ & $\mathrm{O}$ & $\mathrm{O}$ & O \\
\hline I get comfort and understanding from someone. & $\mathrm{O}$ & $\mathrm{O}$ & $\mathrm{O}$ & $\mathrm{O}$ \\
\hline I give up the attempt to cope. & $\mathrm{O}$ & $\mathrm{O}$ & $\mathrm{O}$ & $\mathrm{O}$ \\
\hline I look for something good in what is happening. & $\mathrm{O}$ & $\mathrm{O}$ & $\mathrm{O}$ & $\mathrm{O}$ \\
\hline I make jokes about it. & $\mathrm{O}$ & $\mathrm{O}$ & $\mathrm{O}$ & $\mathrm{O}$ \\
\hline $\begin{array}{l}\text { I do something to think about it less, such as going to movies, } \\
\text { watching TV, reading, daydreaming, sleeping, or shopping. }\end{array}$ & $\mathrm{O}$ & $\mathrm{O}$ & $\mathrm{O}$ & $\mathrm{O}$ \\
\hline I accept the reality of the fact that it has happened. & $\mathrm{O}$ & $\mathrm{O}$ & $\mathrm{O}$ & $\mathrm{O}$ \\
\hline I express my negative feelings. & $\mathrm{O}$ & $\mathrm{O}$ & $\mathrm{O}$ & $\mathrm{O}$ \\
\hline I try to find comfort in my religion or spiritual beliefs. & $\mathrm{O}$ & $\mathrm{O}$ & $\mathrm{O}$ & $\mathrm{O}$ \\
\hline I try to get advice or help from other people about what to do. & $\mathrm{O}$ & $\mathrm{O}$ & $\mathrm{O}$ & $\mathrm{O}$ \\
\hline I learn to live with it. & $\mathrm{O}$ & $\mathrm{O}$ & $\mathrm{O}$ & $\mathrm{O}$ \\
\hline I think hard about what steps to take. & $\mathrm{O}$ & $\mathrm{O}$ & $\mathrm{O}$ & $\mathrm{O}$ \\
\hline I blame myself for things that happen. & $\mathrm{O}$ & $\mathrm{O}$ & $\mathrm{O}$ & $\mathrm{O}$ \\
\hline I pray or meditate. & $\mathrm{O}$ & $\mathrm{O}$ & $\mathrm{O}$ & $\mathrm{O}$ \\
\hline I make fun of the situation. & O & $\mathrm{O}$ & $\mathrm{O}$ & $\mathrm{O}$ \\
\hline
\end{tabular}

If you report suicidal thoughts or behaviors, we encourage you to please take the time to talk to a tribal society member or tribal school counselor.

33. Have you EVER seriously thought about committing suicide?

O Yes

No (If No, go to question \#33.)

33a. If yes, how often have you seriously thought about it in the LAST 12 MONTHS?

Not at All

O A Few Times

O Several Times

O Often 
34. Have you EVER planned how you would commit suicide?

O Yes

No (If No, go to question \#34.)

34a. If yes, have you made a plan for committing suicide in the LAST 12 MONTHS?

O Yes

O No

35. Have you EVER told someone that you were thinking about committing suicide?

O Yes

No (If No, go to question \#35.)

35a. If yes, who did you tell? (Check all that apply)

a Family Member

ㄱ Friends/Peers

a Co-worker

ㄱ Trusted Confidant (e.g., teacher, coach, etc)

․ Service provider (counselor, nurse, healer, doctor)

을 (Please describe.)

35b. In the LAST 12 MONTHS, have you told someone you were thinking about committing suicide?

O Yes

No (If No, go to question \#34.)

35c. If yes, who did you tell?

O Family Member

Friends/Peers

O Co-worker

O Trusted Confidant (e.g., teacher, coach, etc)

Service provider (counselor, nurse, healer, doctor)

Other (Please describe.)

36. Have you EVER attempted suicide?

O Yes

No (If No, go to question \#36.)

36a. If yes, how many times have you attempted suicide IN YOUR LIFETIME? 
36b. If yes, how did you try to kill yourself?

O Shooting

Stabbing or cutting

O Overdose

Hanging

O Causing an Accident

Jumping from Height

Other (Please describe.)

36c. If yes, why did you try to kill yourself?

36d. Have you attempted suicide in the LAST 12 MONTHS?

O Yes

No (If No, go to question \#75.)

36e. How you attempted suicide in the LAST 6 MONTHS?

O Yes

No 
37. This set of questions is about traumatic events that may have happened to you or your loved ones. Please indicate whether you have experienced each event IN YOUR LIFETIME. If you answer yes, then please indicate the NUMBER OF TIME it happened to you and YOUR AGE when the event FIRST OCCURRED.

\begin{tabular}{|c|c|c|c|c|}
\hline & \multicolumn{2}{|c|}{$\begin{array}{l}\text { Experienced } \\
\text { Event in } \\
\text { Lifetime }\end{array}$} & \multirow{2}{*}{$\begin{array}{l}\text { Number of } \\
\text { Times } \\
\text { Occurred } \\
\#\end{array}$} & \multirow{2}{*}{$\begin{array}{l}\text { Age First } \\
\text { Occurred } \\
\text { Age }\end{array}$} \\
\hline & Yes & No & & \\
\hline $\begin{array}{l}\text { 1. Were you ever in a disaster - for example, a flood or flash flood, tornado, fire, drought, or } \\
\text { explosion? }\end{array}$ & O & ○ & & \\
\hline 2. Were you ever in a life-threatening accident? & $\bigcirc$ & $\mathrm{O}$ & & \\
\hline $\begin{array}{l}\text { 3. Have you ever witnessed a serious accident or disaster where someone else was hurt very } \\
\text { badly or killed? }\end{array}$ & $\bigcirc$ & $\mathrm{O}$ & & \\
\hline 4. Have you ever had direct combat experience in a war? & $\bigcirc$ & $\mathrm{O}$ & & \\
\hline $\begin{array}{l}\text { 5. Have you ever been raped, or did you ever have sex when you didn't want to because } \\
\text { someone forced you in some way, or threatened to harm you if you didn't? (If this happened at a } \\
\text { time when you were growing up, it could be just because you thought you had to.) }\end{array}$ & O & ○ & & \\
\hline $\begin{array}{l}\text { 6. Were you ever touched or made to touch someone else in a sexual way because they forced } \\
\text { you in some way, or threatened to harm you if you didn't? }\end{array}$ & $\bigcirc$ & $\bigcirc$ & & \\
\hline 7. Were you ever physically abused or hurt by your parent or a caregiver? & O & O & & \\
\hline 8. Were you ever physically abused or hurt by a spouse or a boyfriend/ girlfriend? & $\mathrm{O}$ & $\mathrm{O}$ & & \\
\hline 9. Were you ever physically abused or hurt by someone else you knew? & O & O & & \\
\hline $\begin{array}{l}\text { 10. Have you ever seen violence between other members of your family? This would include your } \\
\text { family when you were growing up, and your family now. }\end{array}$ & $\mathrm{O}$ & O & & \\
\hline $\begin{array}{l}\text { 11. Have you ever seen your mother being physically abused or hurt by a spouse or } \\
\text { boyfriend/girlfriend? }\end{array}$ & O & O & & \\
\hline $\begin{array}{l}\text { 12. Have you ever seen your father being physically abused or hurt by a spouse or } \\
\text { boyfriend/girlfriend? }\end{array}$ & $\bigcirc$ & $\bigcirc$ & & \\
\hline $\begin{array}{l}\text { 13. Other than the kinds of situations just mentioned, were you ever robbed, mugged, or } \\
\text { physically attacked? (This would not include sexual attacks.) }\end{array}$ & $\bigcirc$ & O & & \\
\hline $\begin{array}{l}\text { 14. Have you ever witnessed someone else being raped, or badly injured or killed? (Other than } \\
\text { the situations you've just described.) }\end{array}$ & $\mathrm{O}$ & O & & \\
\hline $\begin{array}{l}\text { 15. Have you ever had any other experiences like the ones we've been talking about? (IF YES: } \\
\text { Please describe below.) }\end{array}$ & O & O & & \\
\hline 16. Was someone close to you ever in a life threatening situation other than illness? & $\mathrm{O}$ & O & & \\
\hline 17. Was someone close to you ever raped or sexually abused? & O & O & & \\
\hline 18. Has your mother died? & O & $\mathrm{O}$ & & \\
\hline 19. Has your father died? & O & O & & \\
\hline 20. Did a family member or someone close to you ever commit suicide? (If No, go to \#21.) & $\mathrm{O}$ & $\mathrm{O}$ & & \\
\hline $\begin{array}{l}\text { 20a. If YES, at the time, did you believe that you or someone else could be killed or seriously } \\
\text { harmed? }\end{array}$ & O & O & & \\
\hline $\begin{array}{l}\text { 20b. If YES, at the time, were you seriously harmed or was anyone else killed or seriously } \\
\text { harmed? }\end{array}$ & $\mathrm{O}$ & $\mathrm{O}$ & & \\
\hline 21. Was a family member or someone close to you ever murdered? & $\mathrm{O}$ & O & & \\
\hline 22. Did a family member or someone close to you ever die in a car crash? & $\bigcirc$ & $\mathrm{O}$ & & \\
\hline 23. Please describe any other traumatic even you may have experienced. & O & O & & \\
\hline
\end{tabular}


38. If you answered YES to any of the traumatic events listed above, please answer the following questions. The following are symptoms that people sometimes have after experiencing, witnessing, or being confronted with a traumatic event. Please read each one carefully and respond according to how much the symptoms have bothered you since the traumatic event/or the most recent traumatic event noted above. If you report any of the PTSD symptoms listed below, we encourage you to please take the time to talk to a tribal society member or tribal school counselor.

\begin{tabular}{|c|c|c|c|c|}
\hline & Not at All & Rarely & Sometimes & $\begin{array}{l}\text { Most of the } \\
\text { Time }\end{array}$ \\
\hline Recurrent thoughts or memories of the event & $\bigcirc$ & $\mathrm{O}$ & $\bigcirc$ & $\bigcirc$ \\
\hline $\begin{array}{l}\text { Feelings as though the event is happening } \\
\text { again }\end{array}$ & $\bigcirc$ & $\mathrm{O}$ & $\mathrm{O}$ & $\bigcirc$ \\
\hline Recurrent nightmares about the event & $\mathrm{O}$ & $\mathrm{O}$ & O & $\mathrm{O}$ \\
\hline $\begin{array}{l}\text { Sudden emotional or physical reactions when } \\
\text { reminded of the event }\end{array}$ & O & $\mathrm{O}$ & O & $\mathrm{O}$ \\
\hline $\begin{array}{l}\text { Avoiding activities that remind you of the } \\
\text { event }\end{array}$ & $\mathrm{O}$ & $\mathrm{O}$ & $\mathrm{O}$ & O \\
\hline $\begin{array}{l}\text { Avoiding thoughts or feelings associated with } \\
\text { the event }\end{array}$ & $\mathrm{O}$ & $\mathrm{O}$ & $\mathrm{O}$ & $\bigcirc$ \\
\hline Feeling jumpy, easily startled & O & $\mathrm{O}$ & $\mathrm{O}$ & $\mathrm{O}$ \\
\hline Feeling on guard & 0 & $\mathrm{O}$ & $\mathrm{O}$ & $\mathrm{O}$ \\
\hline
\end{tabular}

39. Over the PAST 6 MONTHS, how often have you been bothered by the following problems?

\begin{tabular}{|c|c|c|c|c|}
\hline & Not at All & Several Days & $\begin{array}{l}\text { More than Half } \\
\text { the Days }\end{array}$ & $\begin{array}{l}\text { Nearly Every } \\
\text { Day }\end{array}$ \\
\hline Feeling nervous, anxious or on edge & $\mathrm{O}$ & O & $\mathrm{O}$ & $\bigcirc$ \\
\hline Not being able to stop or control worrying & O & O & O & $\bigcirc$ \\
\hline Worrying too much about different things & $\mathrm{O}$ & $\mathrm{O}$ & $\mathrm{O}$ & $\mathrm{O}$ \\
\hline Trouble relaxing & O & O & $\mathrm{O}$ & O \\
\hline Being so restless that it is hard to sit still & O & O & $\mathrm{O}$ & O \\
\hline Becoming easily annoyed or & O & O & O & O \\
\hline $\begin{array}{l}\text { Feeling afraid as if something awful might } \\
\text { happen }\end{array}$ & $\bigcirc$ & $\bigcirc$ & $\bigcirc$ & 0 \\
\hline
\end{tabular}

40. Has anyone close to you ever died?

O Yes

No (If No, go to question \#41) 
40a. The following statements ask about different thoughts and feelings many people have when they are dealing with the death(s) of someone they have cared about. THINKING ABOUT THE DEATH THAT HAS BEEN HARDEST FOR YOU TO DEAL WITH for each statement, mark the response that tells how often it has happened during the PAST MONTH (4 WEEKS). Please answer every question that you can.

\begin{tabular}{|c|c|c|c|c|c|}
\hline & Never & Rarely & Sometimes & Often & $\begin{array}{l}\text { Almost } \\
\text { Always }\end{array}$ \\
\hline I miss the person who died. & $\mathrm{O}$ & $\mathrm{O}$ & $\mathrm{O}$ & $\mathrm{O}$ & $\mathrm{O}$ \\
\hline $\begin{array}{l}\text { I don't do things because they remind me of how the } \\
\text { person died. }\end{array}$ & $\mathrm{O}$ & $\mathrm{O}$ & $\mathrm{O}$ & $\mathrm{O}$ & $\mathrm{O}$ \\
\hline I enjoy good memories of him/her. & $\mathrm{O}$ & $\mathrm{O}$ & $\mathrm{O}$ & $\mathrm{O}$ & $\mathrm{O}$ \\
\hline $\begin{array}{l}\text { Hearing someone talk about the person makes me think of } \\
\text { the way he/she died. }\end{array}$ & $\mathrm{O}$ & $\mathrm{O}$ & $\mathrm{O}$ & $\mathrm{O}$ & $\mathrm{O}$ \\
\hline I feel like a big part of me has died with him/her. & $\mathrm{O}$ & $\mathrm{O}$ & $\mathrm{O}$ & $\mathrm{O}$ & $\mathrm{O}$ \\
\hline I feel sad about the person's death. & $\mathrm{O}$ & $\mathrm{O}$ & $\mathrm{O}$ & $\mathrm{O}$ & $\mathrm{O}$ \\
\hline I feel guilty about the person's death. & $\mathrm{O}$ & $\mathrm{O}$ & $\mathrm{O}$ & $\mathrm{O}$ & $\mathrm{O}$ \\
\hline Life for me doesn't have much purpose since he/she died. & $\mathrm{O}$ & $\mathrm{O}$ & $\mathrm{O}$ & $\mathrm{O}$ & $\mathrm{O}$ \\
\hline $\begin{array}{l}\text { Upsetting thoughts about how the person died keep me } \\
\text { from enjoying good memories of him/her. }\end{array}$ & $\mathrm{O}$ & $\mathrm{O}$ & $\mathrm{O}$ & $\mathrm{O}$ & $\mathrm{O}$ \\
\hline I get angry when I think about the person who died. & $\mathrm{O}$ & $\mathrm{O}$ & $\mathrm{O}$ & $\mathrm{O}$ & $\mathrm{O}$ \\
\hline My life feels empty without him/her. & $\mathrm{O}$ & $\mathrm{O}$ & $\mathrm{O}$ & $\mathrm{O}$ & $\mathrm{O}$ \\
\hline $\begin{array}{l}\text { I try not to think about the person because I get upset } \\
\text { remembering how he/she died. }\end{array}$ & $\mathrm{O}$ & $\mathrm{O}$ & $\mathrm{O}$ & $\mathrm{O}$ & $\mathrm{O}$ \\
\hline
\end{tabular}


41. If you report any of the symptoms of depression or anxiety listed below, we encourage you to please take the time to talk to a tribal society member or tribal school counselor. Below is a list of the ways you might have felt or behaved. Please tell me how often you have felt this way in the PAST 6 MONTHS.

\begin{tabular}{|c|c|c|c|c|}
\hline & $\begin{array}{l}\text { Rarely or } \\
\text { None of the } \\
\text { Time }\end{array}$ & $\begin{array}{l}\text { Some of or A } \\
\text { Little of the } \\
\text { Time }\end{array}$ & $\begin{array}{l}\text { Occasionally or a } \\
\text { Moderate Amount } \\
\text { of Time }\end{array}$ & $\begin{array}{l}\text { Most or All of } \\
\text { the Time }\end{array}$ \\
\hline $\begin{array}{l}\text { I felt that I could not shake off the blues, even } \\
\text { with the help of my family and friends. }\end{array}$ & $\mathrm{O}$ & $\mathrm{O}$ & $\mathrm{O}$ & $\mathrm{O}$ \\
\hline I felt depressed. & $\mathrm{O}$ & $\mathrm{O}$ & $\mathrm{O}$ & $\mathrm{O}$ \\
\hline I thought my life had been a failure. & $\mathrm{O}$ & $\mathrm{O}$ & $\mathrm{O}$ & $\mathrm{O}$ \\
\hline I felt fearful. & O & $\mathrm{O}$ & $\mathrm{O}$ & O \\
\hline I felt lonely. & $\mathrm{O}$ & $\mathrm{O}$ & $\mathrm{O}$ & $\mathrm{O}$ \\
\hline I had crying spells. & $\mathrm{O}$ & $\mathrm{O}$ & $\mathrm{O}$ & $\mathrm{O}$ \\
\hline I felt sad. & $\mathrm{O}$ & $\mathrm{O}$ & $\mathrm{O}$ & $\mathrm{O}$ \\
\hline $\begin{array}{l}\text { I was bothered by things that usually don't bother } \\
\text { me. }\end{array}$ & $\mathrm{O}$ & $\mathrm{O}$ & $\mathrm{O}$ & $\mathrm{O}$ \\
\hline I did not feel like eating; my appetite was poor. & $\mathrm{O}$ & $\mathrm{O}$ & $\mathrm{O}$ & $\mathrm{O}$ \\
\hline I felt I was just as good as other people. & $\mathrm{O}$ & $\mathrm{O}$ & $\mathrm{O}$ & $\mathrm{O}$ \\
\hline $\begin{array}{l}\text { I had trouble keeping my mind on what I was } \\
\text { doing. }\end{array}$ & $\mathrm{O}$ & $\mathrm{O}$ & $\mathrm{O}$ & $\mathrm{O}$ \\
\hline I felt that everything I did was an effort. & $\mathrm{O}$ & $\mathrm{O}$ & $\mathrm{O}$ & $\mathrm{O}$ \\
\hline I felt hopeful about the future. & $\mathrm{O}$ & $\mathrm{O}$ & $\mathrm{O}$ & $\mathrm{O}$ \\
\hline My sleep was restless. & $\mathrm{O}$ & $\mathrm{O}$ & $\mathrm{O}$ & $\mathrm{O}$ \\
\hline I was happy. & $\mathrm{O}$ & $\mathrm{O}$ & $\mathrm{O}$ & $\mathrm{O}$ \\
\hline I talked less than usual. & $\mathrm{O}$ & $\mathrm{O}$ & $\mathrm{O}$ & $\mathrm{O}$ \\
\hline People were unfriendly. & $\mathrm{O}$ & $\mathrm{O}$ & $\mathrm{O}$ & $\mathrm{O}$ \\
\hline I enjoyed life. & $\mathrm{O}$ & $\mathrm{O}$ & $\mathrm{O}$ & $\mathrm{O}$ \\
\hline I felt that people dislike me. & $\mathrm{O}$ & $\mathrm{O}$ & $\mathrm{O}$ & $\mathrm{O}$ \\
\hline I could not get "going." & $\mathrm{O}$ & $\mathrm{O}$ & $\mathrm{O}$ & $\mathrm{O}$ \\
\hline
\end{tabular}


42. You're almost done! We would like to look at some of your positive strengths. The next series of questions asks about gratitude and resilience.

\begin{tabular}{|c|c|c|c|c|c|c|c|}
\hline & $\begin{array}{l}\text { Strongly } \\
\text { Disagree }\end{array}$ & Disagree & $\begin{array}{l}\text { Somewhat } \\
\text { Disagree }\end{array}$ & $\begin{array}{l}\text { Neither } \\
\text { Agree nor } \\
\text { Disagree }\end{array}$ & $\begin{array}{l}\text { Somewhat } \\
\text { Agree }\end{array}$ & Agree & $\begin{array}{l}\text { Strongly } \\
\text { Agree }\end{array}$ \\
\hline $\begin{array}{l}\text { I have so much in life to be thankful } \\
\text { for. }\end{array}$ & O & O & O & O & O & O & O \\
\hline $\begin{array}{l}\text { If I had to list everything that I felt } \\
\text { grateful for, it would be a very long } \\
\text { list. }\end{array}$ & 0 & 0 & O & O & 0 & O & O \\
\hline $\begin{array}{l}\text { When I look at the world, I don't } \\
\text { see much to be grateful for. }\end{array}$ & O & O & O & O & O & O & O \\
\hline $\begin{array}{l}\text { I am grateful to a wide variety of } \\
\text { people. }\end{array}$ & 0 & 0 & O & O & O & $\mathrm{O}$ & O \\
\hline $\begin{array}{l}\text { I realize I have a lot in my life to } \\
\text { appreciate. }\end{array}$ & 0 & 0 & O & O & O & O & O \\
\hline $\begin{array}{l}\text { Long amounts of time can go by } \\
\text { before I feel grateful for something } \\
\text { or someone. }\end{array}$ & O & O & O & O & O & O & O \\
\hline
\end{tabular}

42a. Please read the following statements. Click the circle that best indicates your feelings about that statement.

\begin{tabular}{|c|c|c|c|c|c|c|c|}
\hline & $\begin{array}{l}\text { Strongly } \\
\text { Disagree }\end{array}$ & Disagree & $\begin{array}{l}\text { Somewhat } \\
\text { Disagree }\end{array}$ & $\begin{array}{l}\text { Neither } \\
\text { Agree nor } \\
\text { Disagree }\end{array}$ & $\begin{array}{l}\text { Somewhat } \\
\text { Agree }\end{array}$ & Agree & $\begin{array}{l}\text { Strongly } \\
\text { Agree }\end{array}$ \\
\hline $\begin{array}{l}\text { I usually manage one way or } \\
\text { another }\end{array}$ & $\mathrm{O}$ & $\mathrm{O}$ & $\mathrm{O}$ & $\mathrm{O}$ & $\mathrm{O}$ & $\mathrm{O}$ & $\mathrm{O}$ \\
\hline $\begin{array}{l}\text { I feel proud that I have } \\
\text { accomplished things in life }\end{array}$ & $\mathrm{O}$ & $\mathrm{O}$ & $\mathrm{O}$ & $\mathrm{O}$ & $\mathrm{O}$ & $\mathrm{O}$ & O \\
\hline I usually take things in stride & $\mathrm{O}$ & $\mathrm{O}$ & $\mathrm{O}$ & $\mathrm{O}$ & $\mathrm{O}$ & $\mathrm{O}$ & $\mathrm{O}$ \\
\hline I am friends with myself & $\mathrm{O}$ & $\mathrm{O}$ & $\mathrm{O}$ & $\mathrm{O}$ & $\mathrm{O}$ & $\mathrm{O}$ & $\mathrm{O}$ \\
\hline $\begin{array}{l}\text { I feel that I can handle many things } \\
\text { at a time }\end{array}$ & $\mathrm{O}$ & $\mathrm{O}$ & $\mathrm{O}$ & $\mathrm{O}$ & $\mathrm{O}$ & $\mathrm{O}$ & $\mathrm{O}$ \\
\hline I am determined & $\mathrm{O}$ & $\mathrm{O}$ & $\mathrm{O}$ & O & $\mathrm{O}$ & $\mathrm{O}$ & $\mathrm{O}$ \\
\hline $\begin{array}{l}\text { I can get through difficult times } \\
\text { because I've experience difficulty } \\
\text { before }\end{array}$ & $\mathrm{O}$ & $\mathrm{O}$ & $\mathrm{O}$ & $\mathrm{O}$ & $\mathrm{O}$ & $\mathrm{O}$ & $\mathrm{O}$ \\
\hline I have self-discipline & $\mathrm{O}$ & $\mathrm{O}$ & $\mathrm{O}$ & $\mathrm{O}$ & $\mathrm{O}$ & $\mathrm{O}$ & $\mathrm{O}$ \\
\hline I keep interested in things & $\mathrm{O}$ & $\mathrm{O}$ & $\mathrm{O}$ & $\mathrm{O}$ & $\mathrm{O}$ & $\mathrm{O}$ & $\mathrm{O}$ \\
\hline $\begin{array}{l}\text { I can usually find something to } \\
\text { laugh about }\end{array}$ & 0 & $\mathrm{O}$ & $\mathrm{O}$ & O & 0 & O & $\mathrm{O}$ \\
\hline $\begin{array}{l}\text { My belief in myself gets me through } \\
\text { hard times }\end{array}$ & $\mathrm{O}$ & $\mathrm{O}$ & $\mathrm{O}$ & $\mathrm{O}$ & $\mathrm{O}$ & $\mathrm{O}$ & $\mathrm{O}$ \\
\hline $\begin{array}{l}\text { In an emergency, I'm someone } \\
\text { people can generally rely on }\end{array}$ & $\mathrm{O}$ & O & $\mathrm{O}$ & O & $\mathrm{O}$ & $\mathrm{O}$ & 0 \\
\hline My life has meaning & $\mathrm{O}$ & $\mathrm{O}$ & $\mathrm{O}$ & $\mathrm{O}$ & $\mathrm{O}$ & $\mathrm{O}$ & $\mathrm{O}$ \\
\hline $\begin{array}{l}\text { When I'm in a difficult situation, I } \\
\text { can usually find my way out of it }\end{array}$ & $\mathrm{O}$ & O & $\mathrm{O}$ & $\mathrm{O}$ & $\mathrm{O}$ & $\mathrm{O}$ & 0 \\
\hline
\end{tabular}


43. Here are the LAST three questions and a place for comments!!

\begin{tabular}{|l|l|l|l|}
\hline & Not at All & Somewhat & A Great Deal \\
\hline Completing this survey made me feel upset. & O & O & O \\
\hline Completing this survey was worth the time. & O & O & O \\
\hline $\begin{array}{l}\text { I felt free to skip questions or to stop at any } \\
\text { time. }\end{array}$ & O & O & O \\
\hline
\end{tabular}

Please feel free to make any comments about the survey or to provide any other important information below.

THANK YOU!!!!!!!!

If you report symptoms of depression, anxiety, or PTSD or suicidal thoughts or behaviors, we encourage you to please take the time to talk to a tribal society member or tribal school counselor.
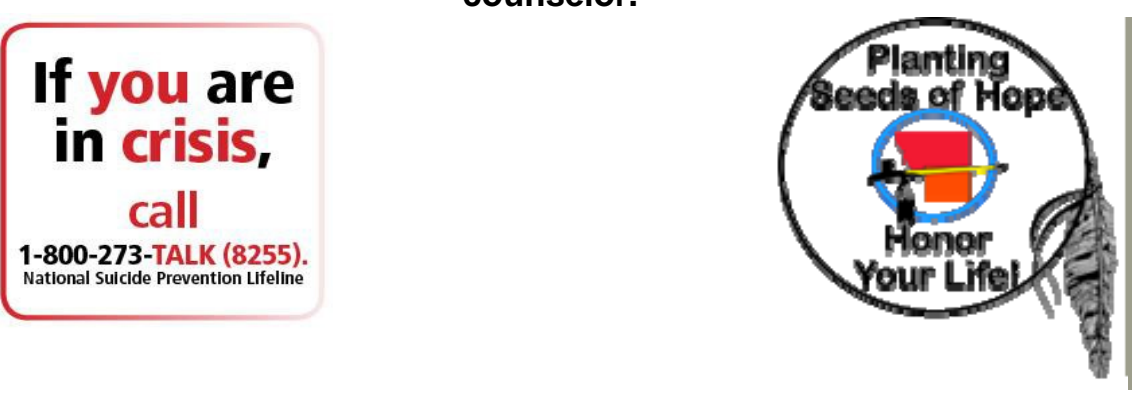